\title{
Multilateration and Kalman Filtering Techniques for Stealth Intelligence Surveillance and Reconnaissance Using Multistatic Radar
}

\author{
by Abdulhak Nagy \\ A thesis \\ presented to the Department of Electronics \\ in fulfillment of the \\ thesis requirement for the degree of \\ Master of Applied Science \\ Ottawa-Carleton Institute for Electrical Engineering \\ Department of Electronics \\ Carleton University \\ Ottawa, Ontario
}




\section{Author's Declaration}

I hereby declare that I am the sole author of this thesis. This is a true copy of the thesis including any required final revisions as accepted by my examiners.

I understand that my thesis may be made electronically available to the public. 


\begin{abstract}
The research presented in this thesis demonstrated that with a multistatic radar in a $2 \mathrm{D}$ plane using the TDOA and FDOA multilateration technique along with the Kalman Filter, to hybrid-geolocate and track a moving stealth target with only two receivers. Hybrid-geolocate and tracking is where the initial location and velocity of the target are unknown. This is an important problem to address because when monitoring boarders between countries, prior knowledge of an incoming target stealth threat is unavailable. Using the Modified Hough Transform, initial and consecutive target locations can be found. Using the dual stage method, reduces the number of receivers required down to two while keeping track of target geolocations. GDOP was used to test boundaries of optimal operation of this radar setup whereas RMSE was used to validate results. This research has shown that in fact hybrid-geolocation and tracking is possible given the dual stage method.
\end{abstract}




\section{Acknowledgements}

I would first like to express my sincere gratitude to my thesis supervisor, Professor Jim Wight, Department of Electronics at Carleton University. Professor Wight's office door was always open whenever I needed to consult on an issue regarding my research or writing. I value his immense patience, expertise and enthusiasm. Without his continual guidance and insightful comments this thesis would not have been possible. I could not have imagined having a better supervisor and mentor for my graduate studies.

Besides my supervisor, I would also like to acknowledge the kind help given to me by department administrator, Ms Blazenka Power.

Finally, I must express my very profound gratitude to my parents, siblings and friends for providing me with unfailing support and continuous encouragement throughout my years of study and through the process of researching and writing this thesis. This accomplishment would not have been possible without them. 


\section{Table of Contents}

Author's Declaration ........................................................................................ ii

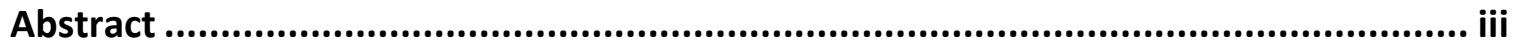

Acknowledgements .................................................................................... iv

Table of Contents ....................................................................................... v

List of Figures ......................................................................................................viii

List of Tables ...............................................................................................

List of Abbreviations..............................................................................................ii

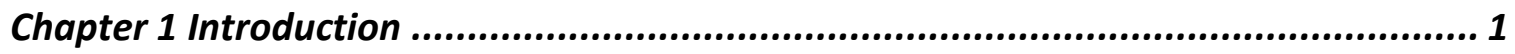

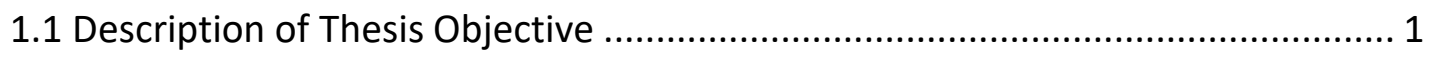

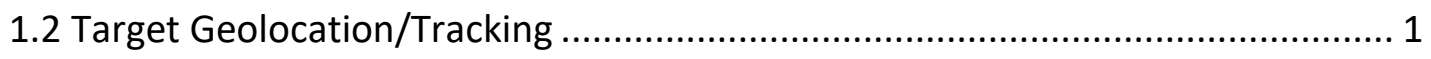

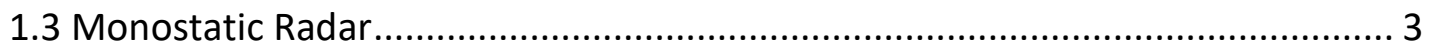

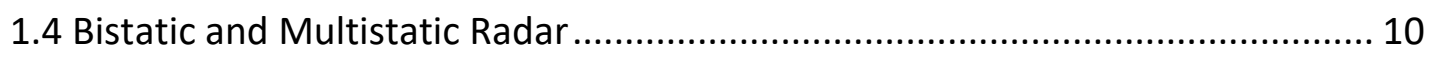

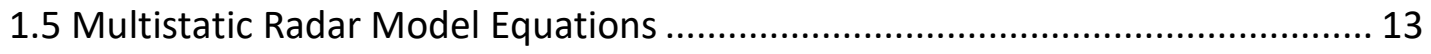

1.6 Comparison between Monostatic and Multistatic Radar ............................... 18

1.7 ISR (Intelligence Surveillance and Reconnaissance) ....................................... 20

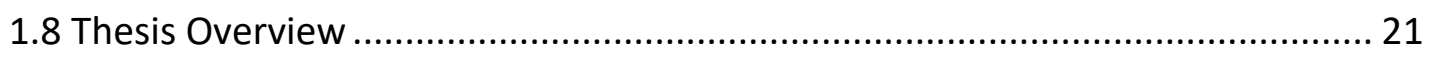

Chapter 2 Multilateration Technique for Geolocation in 2D.................................. 22

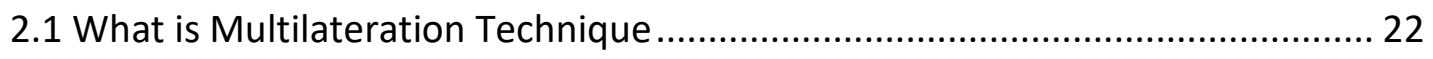

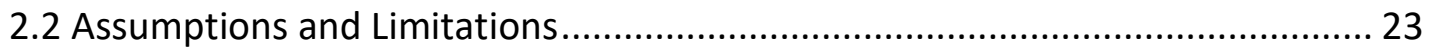

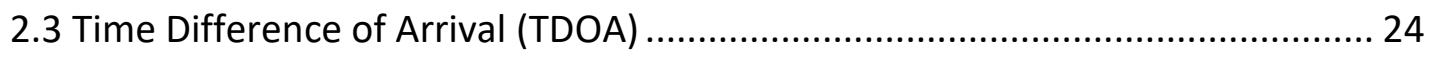

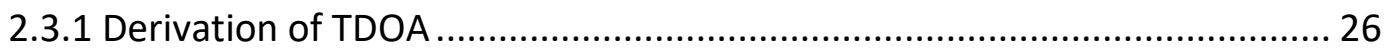

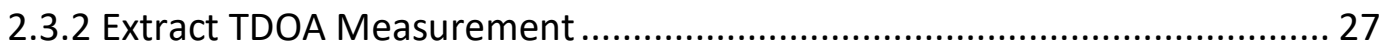

2.4 Frequency Difference of Arrival (FDOA) ..................................................... 35

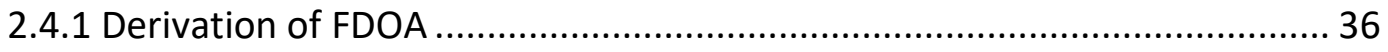

2.4.2 Extract FDOA Measurement ........................................................ 38

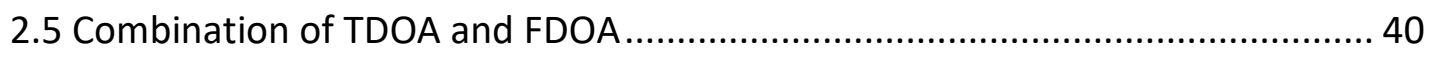

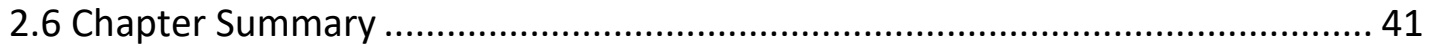




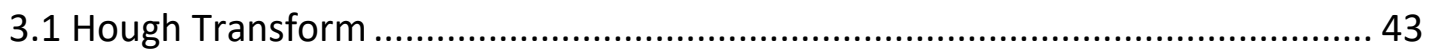

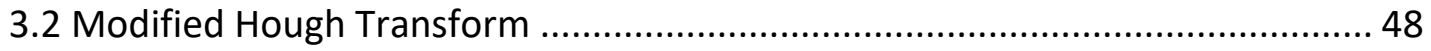

3.3 Data Fusion with Modified Hough Transform .............................................. 51

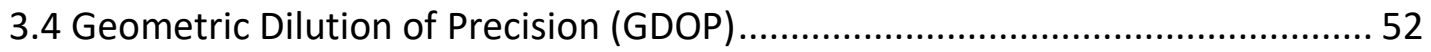

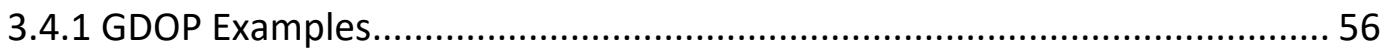

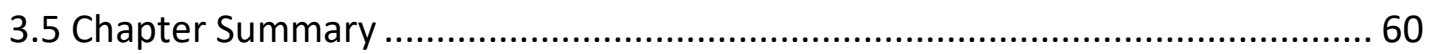

Chapter 4 Filtering Techniques.............................................................. 62

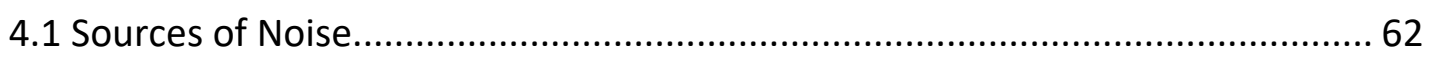

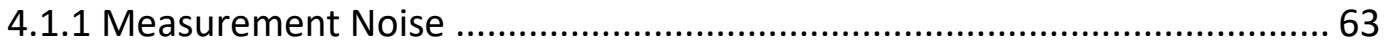

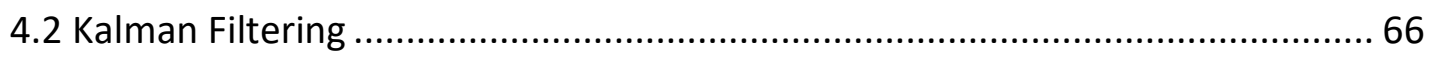

4.2.1 Velocity Prediction with Kalman Filtering............................................. 71

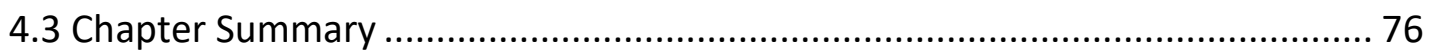

Chapter 5 Radar Simulation on Matlab ..................................................... 77

5.1 Hybrid Geolocation and Tracking Experiment Setup..................................... 77

5.1.1 Parameter and Noise ...................................................................... 77

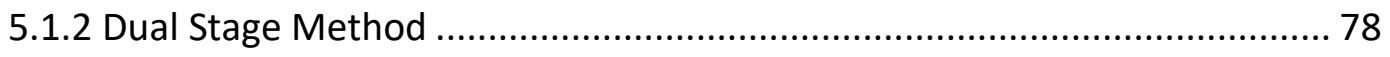

5.2 Time Difference Model for Velocity Prediction ............................................. 81

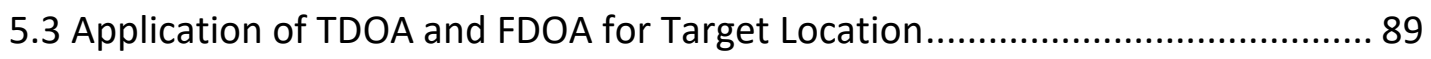

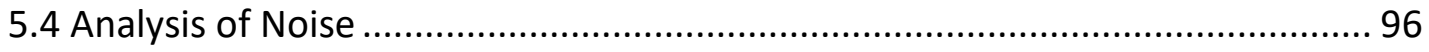

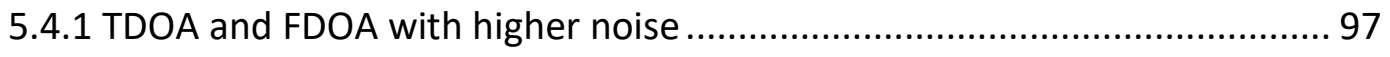

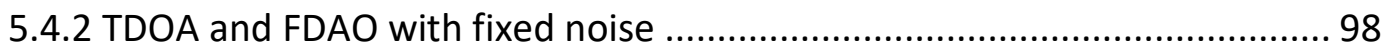

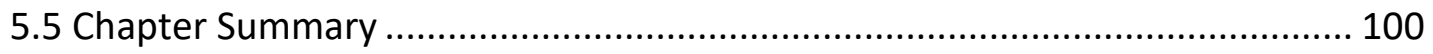

Chapter 6 Conclusion and Recommendations.............................................. 102

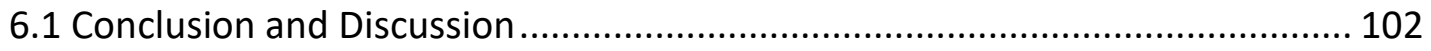




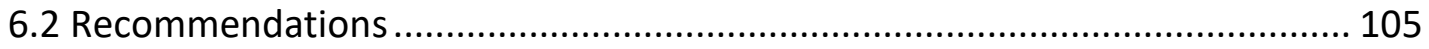

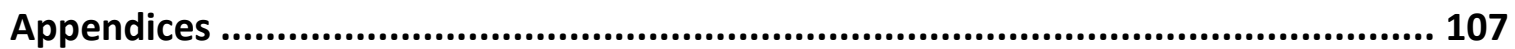

Appendix A Matlab TDOA and FDOA Extraction .............................................. 107

Appendix B Matlab Kalman Filter .......................................................................... 110

Appendix C Matlab Experiment Model .............................................................. 113

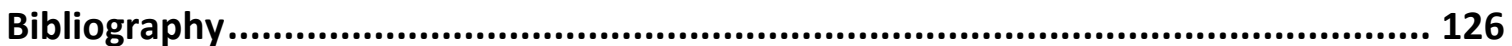




\section{List of Figures}

Figure 1-1 : Diagram represents monostatic radar setup with bistatic angle $>0$ and $<20$

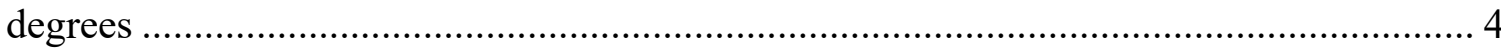

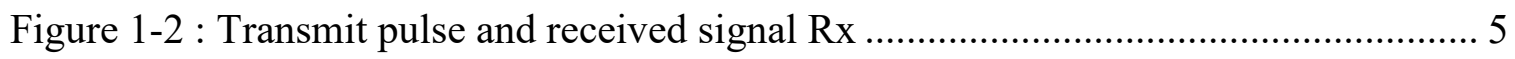

Figure 1-3 : Azimuth and Elevation of target as seen by monostatic radar at $(0,0,0)[11] .6$

Figure 1-4 : Stealth aircraft radar signature with green indicating very low RCS, yellow

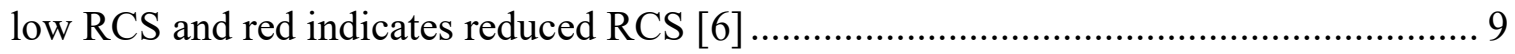

Figure 1-5 : Diagram represents bistatic radar setup with bistatic angle $<20$ degrees [20]

Figure 1-6 : Radar classification between monostatic and bistatic [8] ......................... 13

Figure 1-7 : Geometry for bistatic radar setup [8] ................................................ 15

Figure 1-8 : Constant range sum ellipse with bistatic geometry [7] .......................... 16

Figure 1-9 : Ghost target due to multiple targets under surveillance [2] ....................... 20

Figure 2-1 : Three receivers with 2 LOP intersecting to indicate target location using

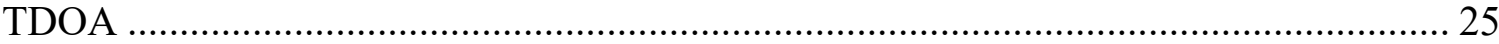

Figure 2-2 : Signal 1 with pulse 200 time units and freq. $20 \mathrm{~Hz}$ starting at 50 time units 28

Figure 2-3 : Signal 2 with pulse 200 time units and freq. $10 \mathrm{~Hz}$ starting at 100 time units

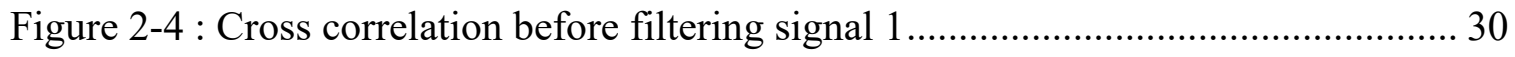

Figure 2-5 : Frequency spectrum after down converting ...................................... 31

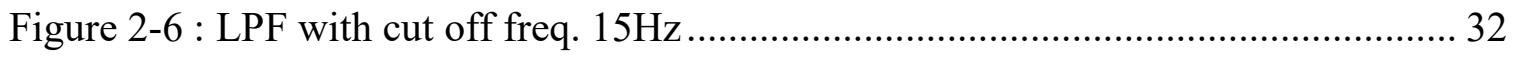

Figure 2-7 : Signal 1 after having it down shifted and filtered with LPF ..................... 33

Figure 2-8 : Cross correlation between signal 1 and 2 with similar frequency ............... 34 
Figure 2-9: Three receivers with 2 LOP intersecting to indicate target location using

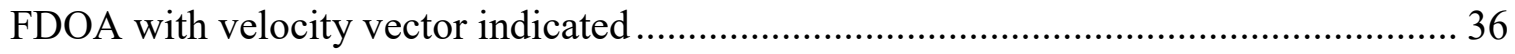

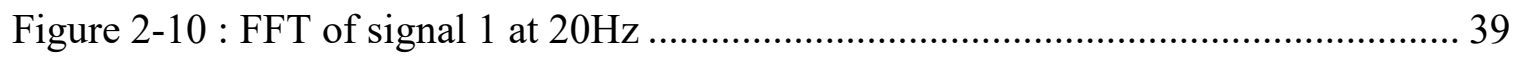

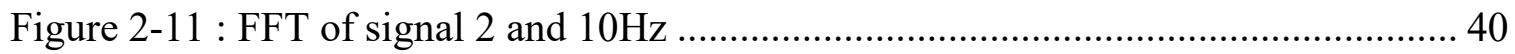

Figure 2-12 : Two receivers with LOP due to TDOA and FDOA............................... 41

Figure 3-1 : Feature Space with 5 points used as samples for the Parameter Space ....... 45

Figure 3-2 : Parameter Space $(\mathrm{m}=1.99, \mathrm{~b}=1.00)$ formed by 5 points taken from Feature

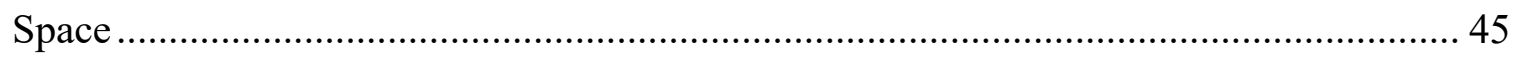

Figure 3-3 : Parameter Space in polar coordinates $(\varnothing=2.68, \mathrm{r}=0.21)$ represented by 5

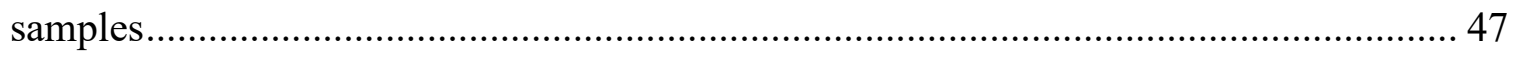

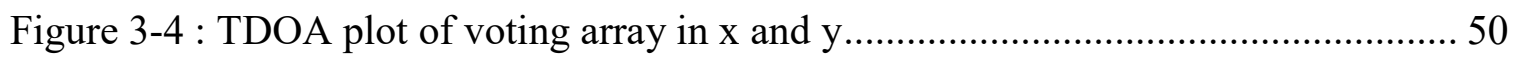

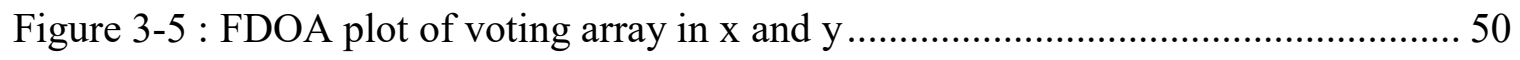

Figure 3-6 : TDOA and FDOA data fusion to form Accumulator Array ....................... 52

Figure 3-7 : Demonstration of crossrange and downrange with 2 receivers [2] ............. 54

Figure 3-8 : Multistatic error eclipse approximated as parallelogram for two virtual

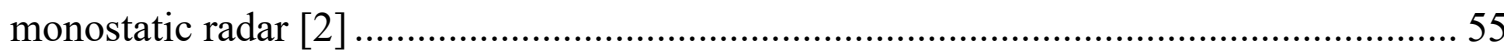

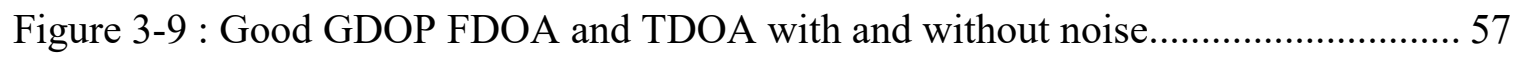

Figure 3-10 : Target located $(1000,500)$ without noise and $(964,468)$ with noise ........... 58

Figure 3-11 : Bad GDOP FDOA and TDOA with and without noise ........................... 58

Figure 3-12 : Target located $(1000,500)$ without noise and $(185,20)$ with noise both with

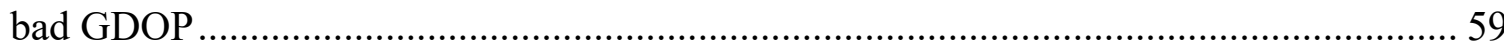

Figure 3-13 : Bad GDOP is more sensitive to noise than Good GDOP setup.................. 60

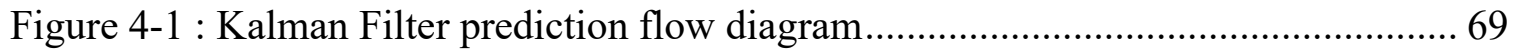


Figure 4-2 : Kalman Filter prediction in one dimension [21] .................................... 71

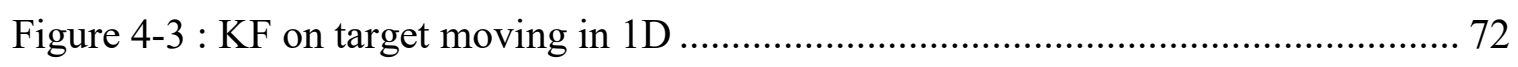

Figure 4-4 : RMSE of Measured Data Location ................................................... 73

Figure 4-5 : RMSE of velocity prediction from the KF........................................... 74

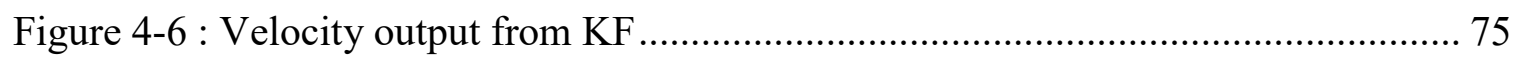

Figure 5-1 : Flow Diagram Indicating the Dual Stage Method .................................... 80

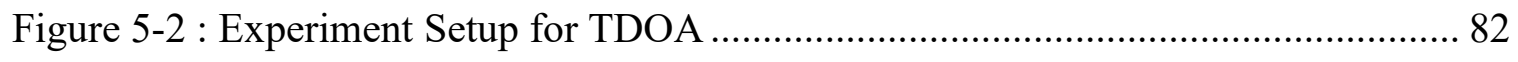

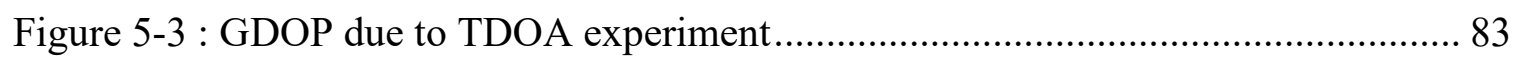

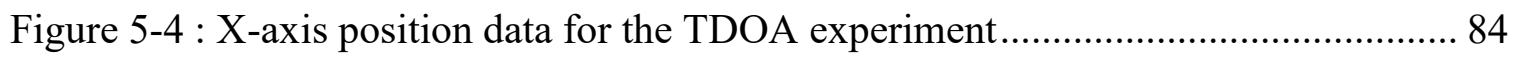

Figure 5-5 : X-axis prediction of target velocity for the TDOA experiment ................... 85

Figure 5-6 : X-axis Root Mean Squared Error for the filtered target velocity ................. 86

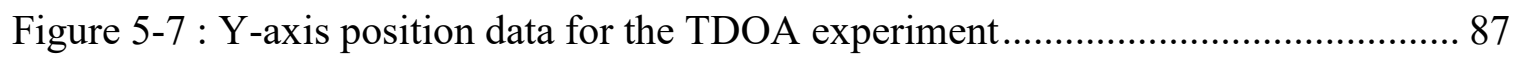

Figure 5-8 : Y-axis prediction of target velocity for the TDOA experiment ................... 88

Figure 5-9 : Y-axis Root Mean Squared Error for the filtered target velocity ................. 89

Figure 5-10 : Experiment Setup for TDOA and FDOA ........................................ 91

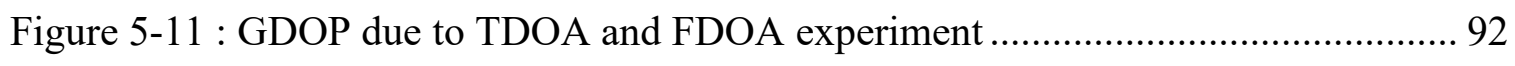

Figure 5-12 : X-axis position data for the TDOA and FDOA experiment ...................... 93

Figure 5-13 : X-axis Root Mean Squared Error for the filtered target location ............... 94

Figure 5-14 : Y-axis position data for the TDOA and FDOA experiment ..................... 95

Figure 5-15 : Y-axis Root Mean Squared Error for the filtered target location ............... 96

Figure 5-16: Y-axis position data for the TDOA and FDOA experiment with higher

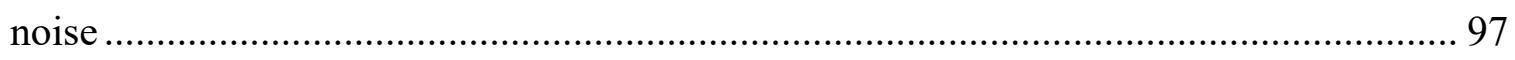


Figure 5-17 : Y-axis Root Mean Squared Error for the filtered target location with higher noise 98

Figure 5-18 : Experimental setup for fixed noise demonstration using TDOA and FDOA

Figure 5-19: Y-axis measured location data for the TDOA and FDOA experiment with

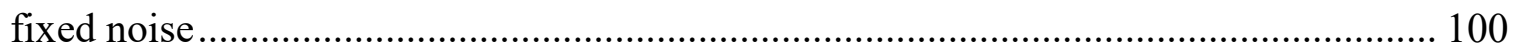




\section{List of Tables}

Table 1-1 : Terminologies of target location (Note: * Target assumed to have constant

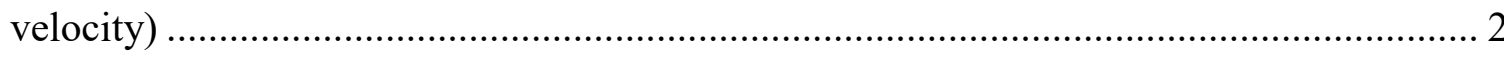

Table 3-1 : GDOP measurements for different multistatic radar setup ........................... 56

Table 4-1 : Experiment setup parameter in 1D ........................................................... 73

Table 5-1 : Constant Experiment Parameters ………………………………………..... 78

Table 5-2 : Parameters for Simulation using TDOA multilateration Technique............... 81

Table 5-3: Parameters for Simulation using TDOA and FDOA multilateration

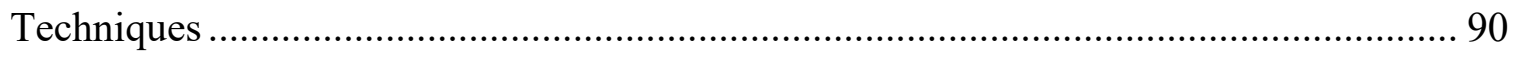




\section{List of Abbreviations}

TDOA Time Difference of Arrival

FDOA Frequency Difference of Arrival

RADAR Radio Detection and Ranging

KF Kalman Filter

DF Direction Finder

dB decibels

LOS Line of Sight

LOP Line of Position

PRI Pulse Repetition Interval

PRF Pulse Repetition Frequency

HT Hough Transform

CW Continuous Wave

FFT Fast Fourier Transform

DFT Discrete Fourier Transform

FM Frequency Modulation

GDOP Geometric Dilution of Precision

GPS Global Positioning System

PDF Probability Distribution Function

RCS Radar Cross Section

SNR Signal to Noise Ratio

MLAT Multilateration

TOA Time of Arrival 
DOA Difference of Arrival

2D 2-Dimension

CS Central Station

EM Electro Magnetic

CRLB Cramer Rao Lower Bound

VHF Very High Frequency (30MHZ-300MHz)

UHF Ultra High Frequency (300MHz-3GHz)

FS Feature Space

PS Parameter Space

BW Band Width

UAV Unmanned Areal Vehicle

RMSE Root Mean Squared Error

PBR Passive Bistatic Radar 


\section{Chapter 1 Introduction}

This section will cover the thesis objective along with the definition of radar in the context of monostatic and multistatic radar.

\subsection{Description of Thesis Objective}

The most common radar used today cannot detect stealth or any other aircraft without them being detected. This is because conventional radars are monostatic, meaning they have the transmitter and receiver co-located in the same place. With stealth aircraft designed to diffract signals in other directions beside the point of origin, the probability of detection by this radar setup is reduced [2]. Furthermore, this allows the enemy to geo-locate the monostatic radar. The solution to this problem is to use bistatic or multistatic radar whereby the multilateration technique such as Time Difference of Arrival (TDOA) and Frequency Difference of Arrival (FDOA) measurements are obtained to help in geolocating a target while remaining invisible.

The purpose of this thesis is to demonstrate that with a multistatic radar in a 2D plane using the TDOA and FDOA multilateration technique along with the Kalman Filter (KF), to hybrid geolocate and track a moving stealth target with only two receivers. Hybrid geolocation and tracking is where initial location and velocity of the target are unknown.

\subsection{Target Geolocation/Tracking}

In order to get a better perspective on the thesis objective, the description of terminologies below will help in clarifying differences and similarities between 
localization, geolocation, tracking and a hybrid of the former two types. Table 1.1 below summarizes the results of the terminologies.

Table 1-1: Terminologies of target location (Note: * Target assumed to have constant velocity)

\begin{tabular}{|l|l|l|l|l|}
\hline Type & $\begin{array}{l}\text { Initial Target } \\
\text { Location }\end{array}$ & Velocity & Mobility & Mobility \\
\hline Localization & unknown & zero & stationary & stationary \\
\hline Geolocation & unknown & zero & stationary & stationary OR \\
& & & mobile \\
\hline Target Tracking & known & known & mobile* & stationary OR \\
& & & mobile \\
\hline Hybrid Geolocation and & unknown & unknown & mobile * & stationary OR \\
& & & & mobile \\
\hline
\end{tabular}

Target localization is where the target and the radar receivers used to locate the position of the target are all stationary. Prior knowledge of the target location is unknown.

Doppler measurements can't be used here as there is no motion of receiving sensor(s) or/and the target.

Target geolocation is defined as locating the target position with the receiving sensor(s) having motion while the target is strictly stationary. There is no prior knowledge of the target location. However, in this scenario Doppler measurements can be used to locate the target as the receivers are in motion with known velocity. 
The next terminology involves prior knowledge of the target location and current target velocity to track the target location while having the capability to use Doppler measurements as the target and sensor velocity are known. Also target trajectory needs to follow a predictable path such that the transition matrix can model this accurately. Transition matrix will be explained in Chapter 4.

The final target location type is the hybrid between geolocation and tracking where the target's initial location and current velocity are unknown. The receivers can be stationary or mobile as their relative velocity can be determined once the target velocity is measured by taking consecutive location measurements of the target. Using the dual stage method, the hybrid geolocation and tracking will be the focus of my thesis.

\subsection{Monostatic Radar}

Monostatic radar is the traditional radar most commonly used in military and civilian applications. The definition of Radio Detection and Ranging (RADAR) is mutual with the monostatic setup. It is an electronic system whereby a radiofrequency (RF) electromagnetic (EM) wave is sent out by an antenna to locate the presence of a target, its distance, direction and speed [8].

Where the receiver and transmitter are co-located in the nearly same area, they are known as quasi-monostatic radar. As seen by the target's frame of reference relative to the transmitter and receiver, the maximum bistatic angle of 20 degrees is measured between the target-transmitter and target-receiver vectors. 
Figure 1-1 demonstrates an example of a monostatic radar setup where the receiver and transmitter are within a bistatic angle of 20 degrees or less. For most pulsed monostatic radars today, the bistatic angle is zero degrees because the same antenna is used as a transceiver meaning it sends out and receives signals using the same antenna. I will make reference to monostatic radar with a bistatic angle of zero degrees.
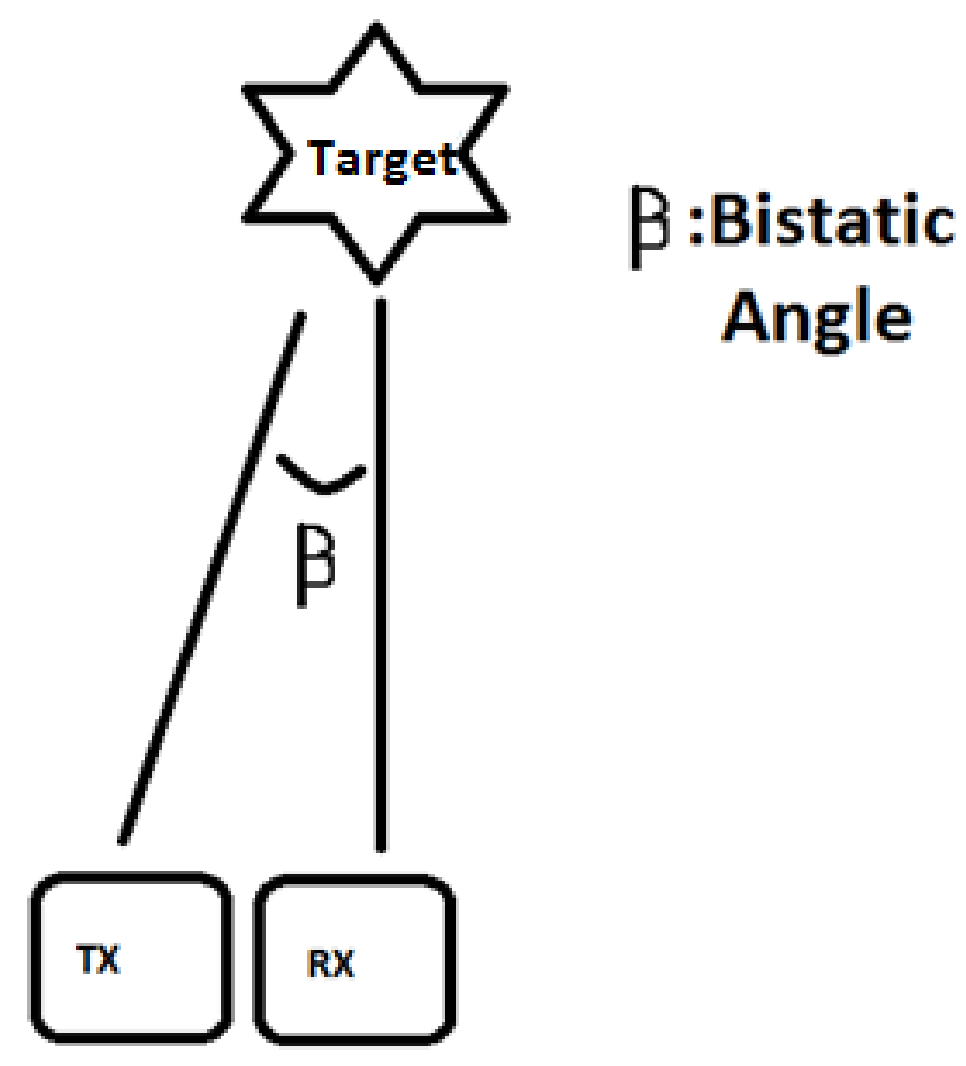

Figure 1-1: Diagram represents monostatic radar setup with bistatic angle $>0$ and $<20$ degrees

The monostatic radar functions by transmitting a pulsed EM wave in the direction of a target and receiving a portion of the reflected EM wave or signal. Signal reflections happen off a target due to EM waves inducing currents on the target. These induced 
currents in turn reradiate back EM waves, some of which are within the bistatic angle of 20 degrees.

The range $\mathrm{R}$ of a target is determined by the round trip time $\Delta t=t 2-t 1$ it takes for the transmitted EM signal at time t1 to propagate to the target and reach back to the receiver at time $\mathrm{t} 2$ in the speed of light $\mathrm{c}$. In this case, the range or distance to the target from the radar will be half the round trip distance. This can be seen in Figure 1-2 where the round trip time is twice the distance from the radar to the target.

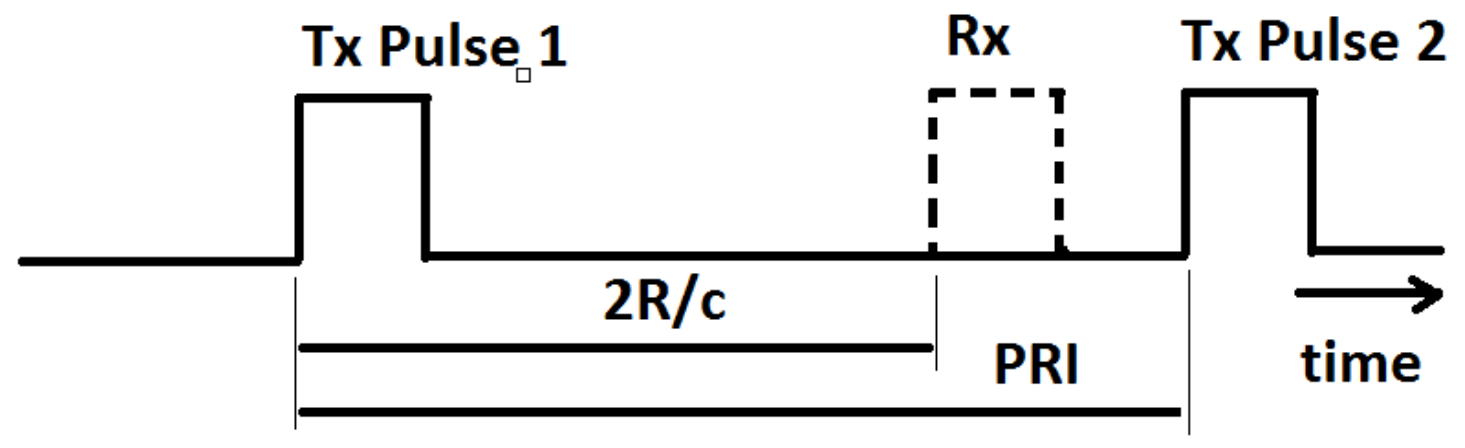

Figure 1-2 : Transmit pulse and received signal $R x$

The range $\mathrm{R}$ from the radar to the target can be measured as shown by equation 1.1 below where $\mathrm{c}$ is the speed of light at which EM waves travel and $\Delta \mathrm{t}$ is the round trip time [5]. $R=\frac{c \Delta t}{2}$

The target direction is obtained by noting the azimuth and elevation angles at which the radar received an echo from a given target using a directional antenna. The azimuth angle is the rotational angle about the vertical axis whereas the elevation angle is the rotational angle about the horizontal axis relative to a flat surface. This is demonstrated in 
Figure 1-3 where the azimuth angle is around the z-axis and the elevation angle is around the $x-y$ plane.

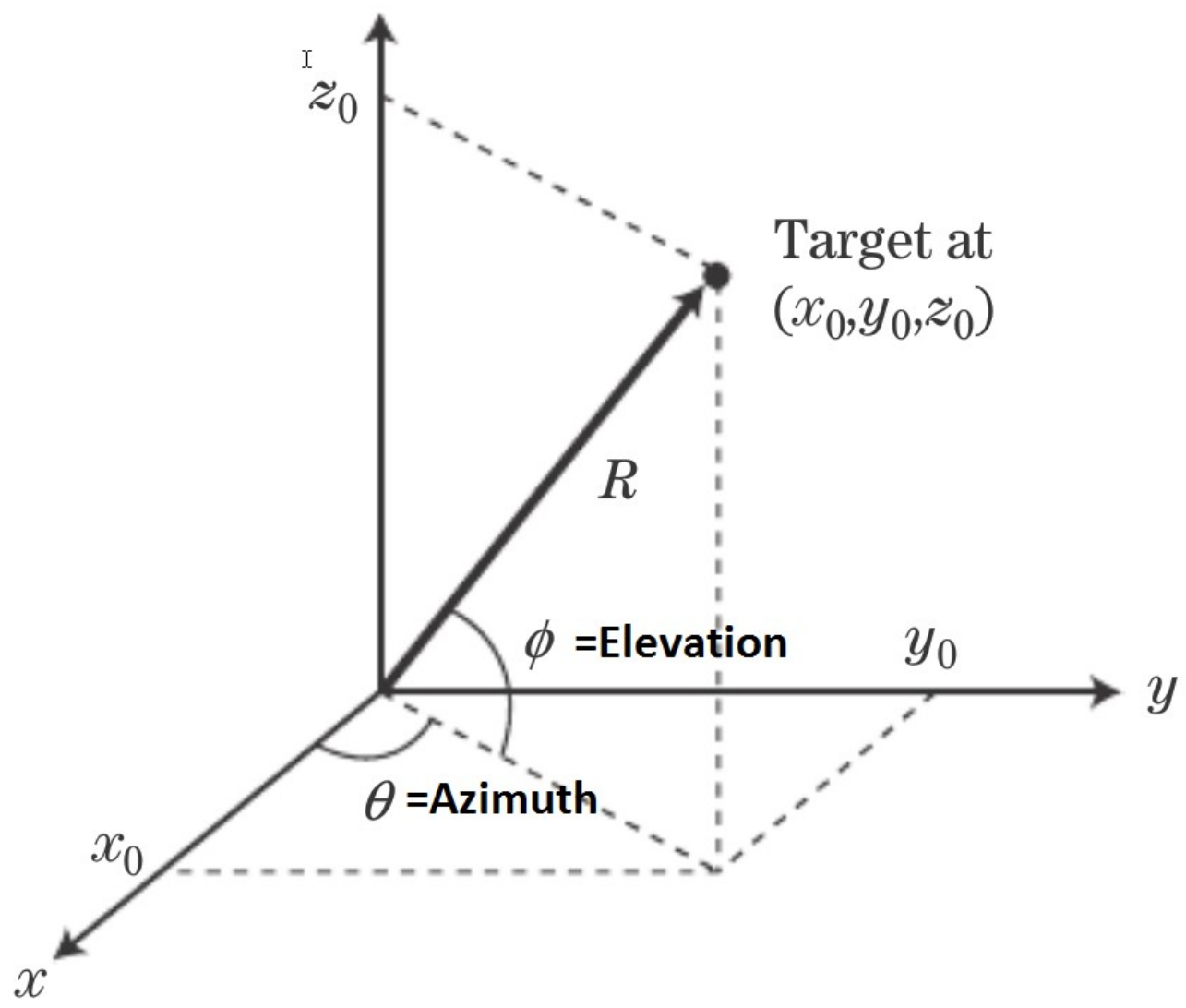

Figure 1-3 : Azimuth and Elevation of target as seen by monostatic radar at $(0,0,0)$ [11]

The speed of a moving target creates a Doppler effect or a frequency shift also known as a beat frequency $f_{m}$ on the reflected signal frequency $f_{r}$ relative to the incident signal frequency $f_{t}$. The frequency shifts detected by the receiver can be identified, if the target is moving away from or approaching the radar; these frequency shifts can also detect the velocity of the target and discriminate between moving targets with beat frequency and stationary targets with zero beat frequency. The beat frequency is approximated by the equation 1.2 where the speed of light is much greater than the target velocity v. 
$f_{m} \approx 2 v \frac{f_{t}}{c}$

The Pulse Repetition Interval (PRI) limits the maximum range that a radar can detect a target before the results are ambiguous, hence it defines the maximum unambiguous range. The PRI is the interval between two consecutive pulses and the rate at which these pulses are sent out from the radar is the Pulse Repetition Frequency (PRF) which is the inverse of the PRI. The unambiguous range is limited by the PRI because in order for the radar to distinguish between echo returns, it needs to know which transmitted pulse it belongs to. So, for example, if an echo is received shortly after sending out a second pulse, the radar would need to determine if the signal belongs to returns from the second or first pulse sent out. This would result in two completely different range calculations using equation 1.1 above. The unambiguous range is identified by equation 1.3 below as a function of PRF.

$R_{U N A M B I G}=\frac{c}{2 * P R F}$

The maximum range of a monostatic or conventional radar is determined by the minimum power the receiver can detect and is given by the equation 1.4 which can resolve both ambiguous and unambiguous targets given further processing.

$R_{\max }^{4}=\frac{P_{t} G_{t} G_{r} \sigma \lambda^{2}}{(4 \pi)^{2} P_{r-\min }}$

where the peak power transmitted is $P_{t}$, the transmit and receive gains respectively are $G_{t}$ and $G_{r}$; the target cross-section is $\sigma$, signal wavelength is $\lambda$ and the minimum received 
power $P_{r-\min }$. Hence, the maximum range $R_{\max }^{4}$ is limited by the minimum received power.

The Radar Cross Section (RCS) $\sigma$ plays a key role in determining how much power will be reflected back to the receiver. When a target has certain stealth capabilities like having a reduced RCS as seen by a monostatic radar, the target appears invisible. This is due to either shape or the radar absorption material used. Shaping deflects signals in directions other than the source and absorption transfers the EM wave to heat [6]. The latter will make the plane prone to heat seeking devices thus focus will be on detecting a stealth target with scattering surface. The RCS of a stealth fighter can be seen in Figure 1-4 with better stealth signatures at higher frequencies; for example, the X-band radar signature shows highest RCS at about 90 degrees relative to the nose. This means that any incident signal's reflection is seen stronger at 90 degrees due to a larger RCS value. 


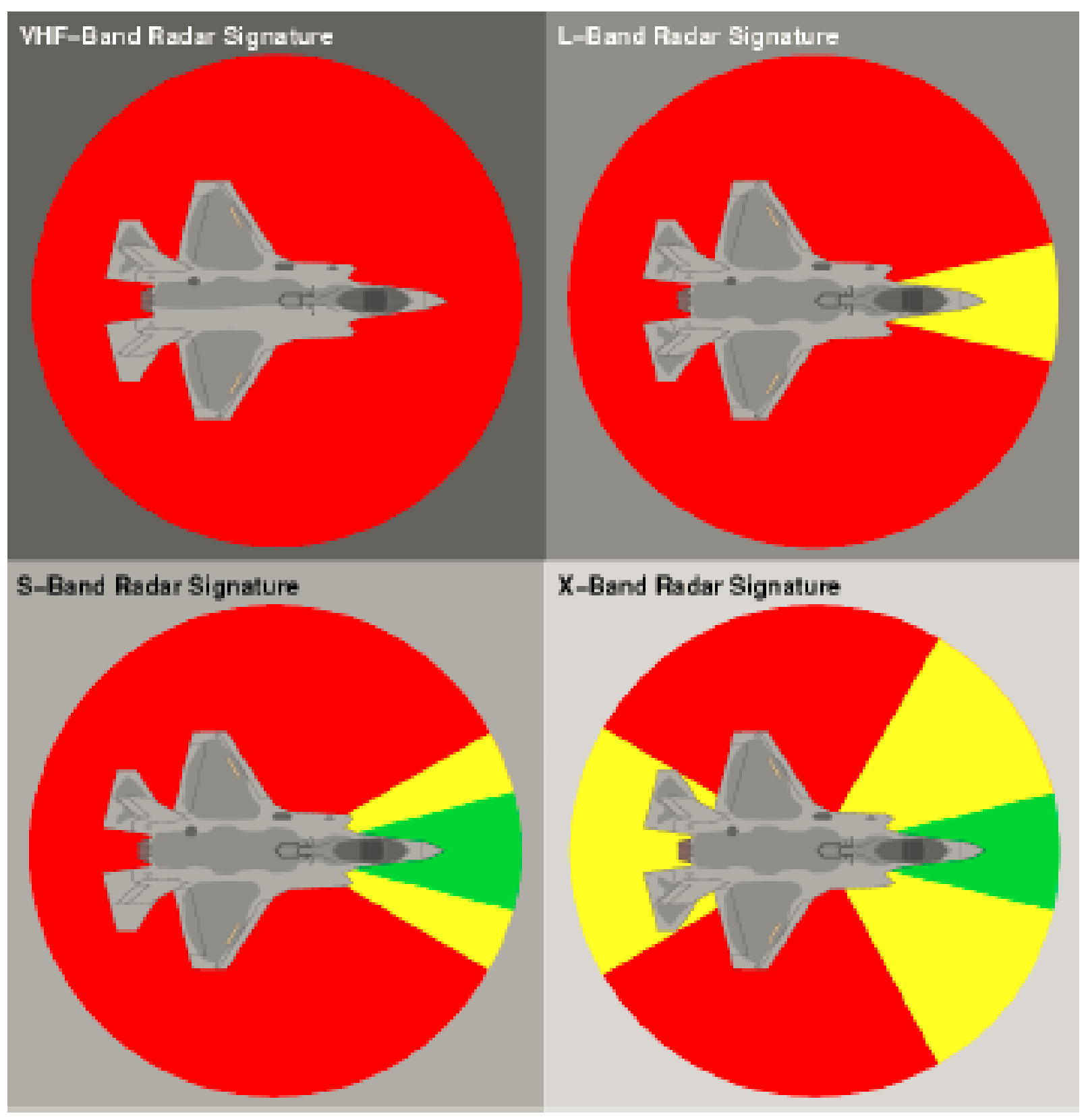

Figure 1-4 : Stealth aircraft radar signature with green indicating very low RCS, yellow low RCS and red indicates reduced RCS [6] 


\subsection{Bistatic and Multistatic Radar}

Put in simple terms, a bistatic radar is where a transmitter and receiver are not co-located in the same area but separated by a distance comparable to an expected target location. The subtended angle between the transmitter target and receiver, also known as a bistatic angle, is typically greater than 20 degrees. Any angle between 145 degrees to 180 degrees is known as forward/fence geometry, which was amongst the first bistatic radar applications. Multistatic radar, on the other hand, involves two or more receivers distributed with one transmitter(s) or vice-versa across a plane of surveillance. [5]

Bistatic radars can use uniquely designed dedicated transmitters for bistatic operations. They can also be operated with transmitters of opportunity, essentially designed for other uses, but still appropriate for bistatic operations. The term 'hitchhicker' is used to identify the bistatic radar if the transmitter of opportunity comes from a monostatic radar. The transmitter of opportunity may be from non-radar sources like a communications link or a broadcast station. In this case, the bistatic radar can also be identified as passive radar, parasitic radar, piggyback radar and passive coherent location. In this thesis I will be referring to bistatic radar that is passive as Passive Bistatic Radar (PBR). In military cases, transmitters of opportunity can be either 'cooperative' or 'noncooperative', with the former designating a friendly transmitter and the latter a hostile one [2].

Target location measurements by the PBR can be significantly improved by abandoning coarse angular measurements in favor of implementing range and/or Doppler multilateration techniques. Then a target's iso-range contours from each transmitter- 
receiver pair can be combined to produce intersecting contours, which locate the target. Alternatively, iso-Doppler contours can be used whenever initial conditions can be satisfied. They can also be integrated with iso-range contours in an attempt to improve location accuracy. This target location is then processed by a tracking algorithm, for example, an alpha-beta tracker or Kalman filter, to generate target state estimates, just as in a traditional monostatic radar [5].

A bistatic radar setup can be seen in Figure 1-5 where the bistatic angle is greater than 20 degrees. By expanding on this figure to represent a multistatic radar by having at least a second receiver or transmitter located anywhere besides the current one. Information gathered from all receivers in either a bistatic or multistatic setup is sent to a central processing unit to compute target location.

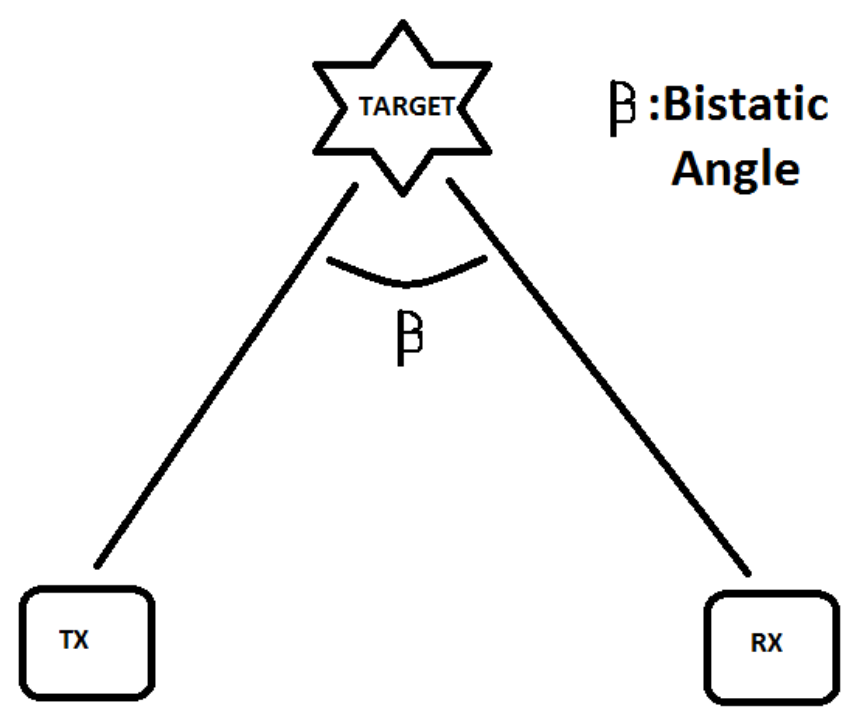

Figure 1-5 : Diagram represents bistatic radar setup with bistatic angle $<20$ degrees [20] 
The various types of radars - monostatic and bistatic, or multistatic - are classified in Figure 1-6. Moreover, while a cooperative transmitter is controlled by the user, the noncooperative one, like a hitchhiker, is from a radio source with the transmitter signal originating from a broadcast, communications or radio navigation signal. There are clearly some benefits of Passive Bistatic Radar (PBR). Firstly, not unlike other bistatic radars, the receiver is passive with the potential of making it undetectable - essentially making it immune to any anti-radiation missile attack. With the adversary not being aware of the receiver's location, any jamming done would have to be non-directional, resulting in the dilution of its effectiveness. Also, with transmitters of opportunity located anywhere within the surveillance region, the adversary does not know you are watching. A counter-stealth benefit may be offered by a bistatic radar system: in bistatic geometries, to the reduced monostatic Radar Cross Section (RCS) of a target, the target shaping may be less effective. Another advantage of bistatic radars could also be the ability to exploit the significant enhancement of a target RCS, occurring in forward scatter. VHF or UHF frequencies, not usually available for radar use, will often be used by PBR systems [8]. Here, RCS reduction techniques may prove less effective than at microwave frequencies. This is because both radar wave length and target dimensions will often be of a similar order. Many high power illumination possibilities exist; the increasing congestion of the electromagnetic spectrum poses a real problem for virtually all radar systems, but is in fact a benefit for PRB. Lastly, receiver system tend to be inexpensive and rather simple with the transmitter not requiring any license. 


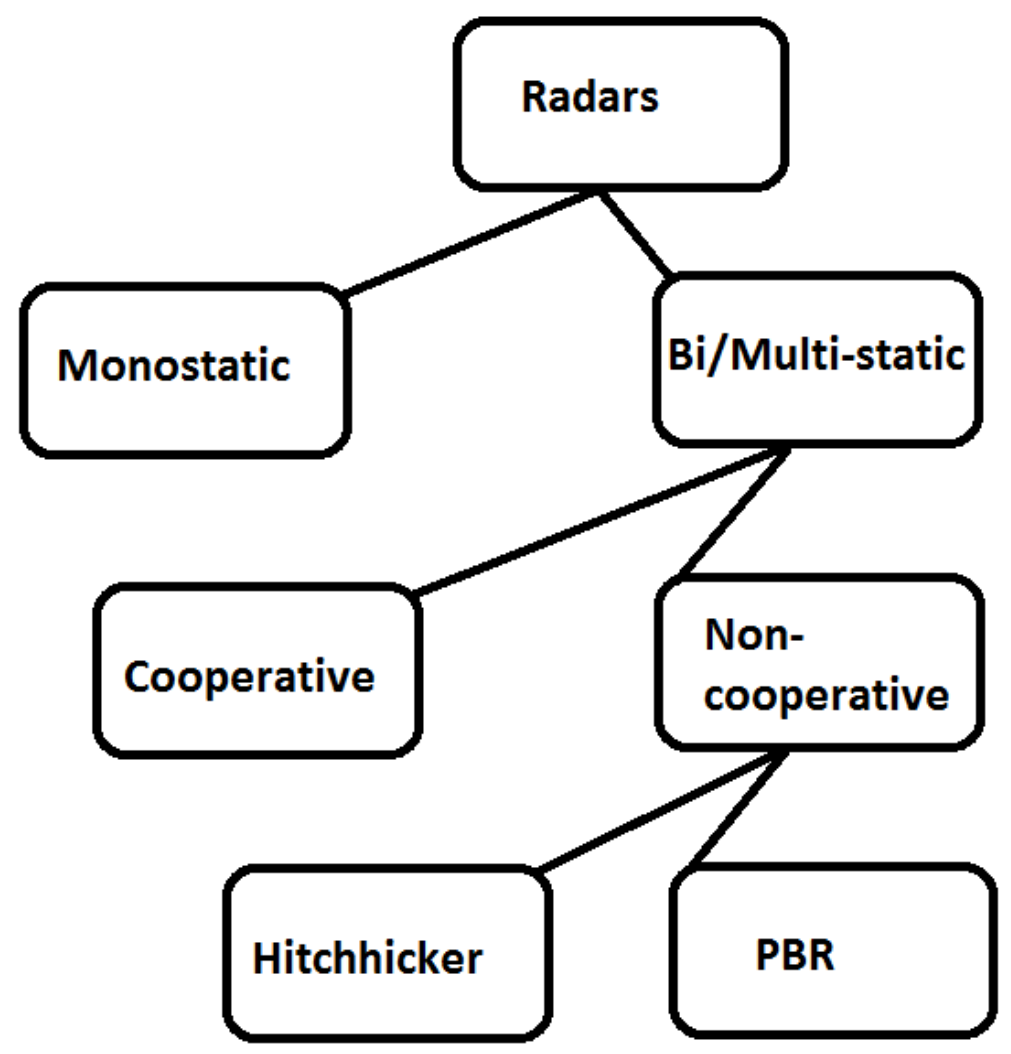

Figure 1-6 : Radar classification between monostatic and bistatic [8]

\subsection{Multistatic Radar Model Equations}

Target detection is performed bistatically: each transmit-receive pair makes independent detections inside a surveillance region, which is common to all such pairs. In target location, typical measurements are made of the baseline and simultaneous range-sum from multiple transmitter-receiver pairs. These are plotted as ellipses with a transmitterreceiver pair at each ellipse foci. The target is located by the intersection of these ellipses, or constant range-sum contours; this resembles multilateration for this reason: the target is located by only using range measurements [5]. 
Multistatic radars often employ expressions, concepts and data specifically developed for bistatic radars; such terms include target Doppler, surface clutter, and target radar cross section. The remainder of this chapter will be devoted to bistatic radar, developing multistatic exceptions when required [5].

Figure 1-7 identifies basic bistatic geometry. The baseline L separates the transmitter and receiver. The bistatic angle, $\beta$ is the angle which is subtended at the target by the transmitter and receiver. The aspect angle, $\delta$ represents the angle from the target velocity vector $\mathrm{V}$ to the bisect of the bistatic angle. The bistatic receiver may measure one of three essential parameters: (a) the difference in range $\left(R_{T}+R_{R}-L\right)$ between the direct 
signal and the transmitter-target-receiver path, (b) the angle of arrival $\theta_{R}$ of the received echo, and (c) the Doppler shift $f_{b}$ of the received echo. [8]

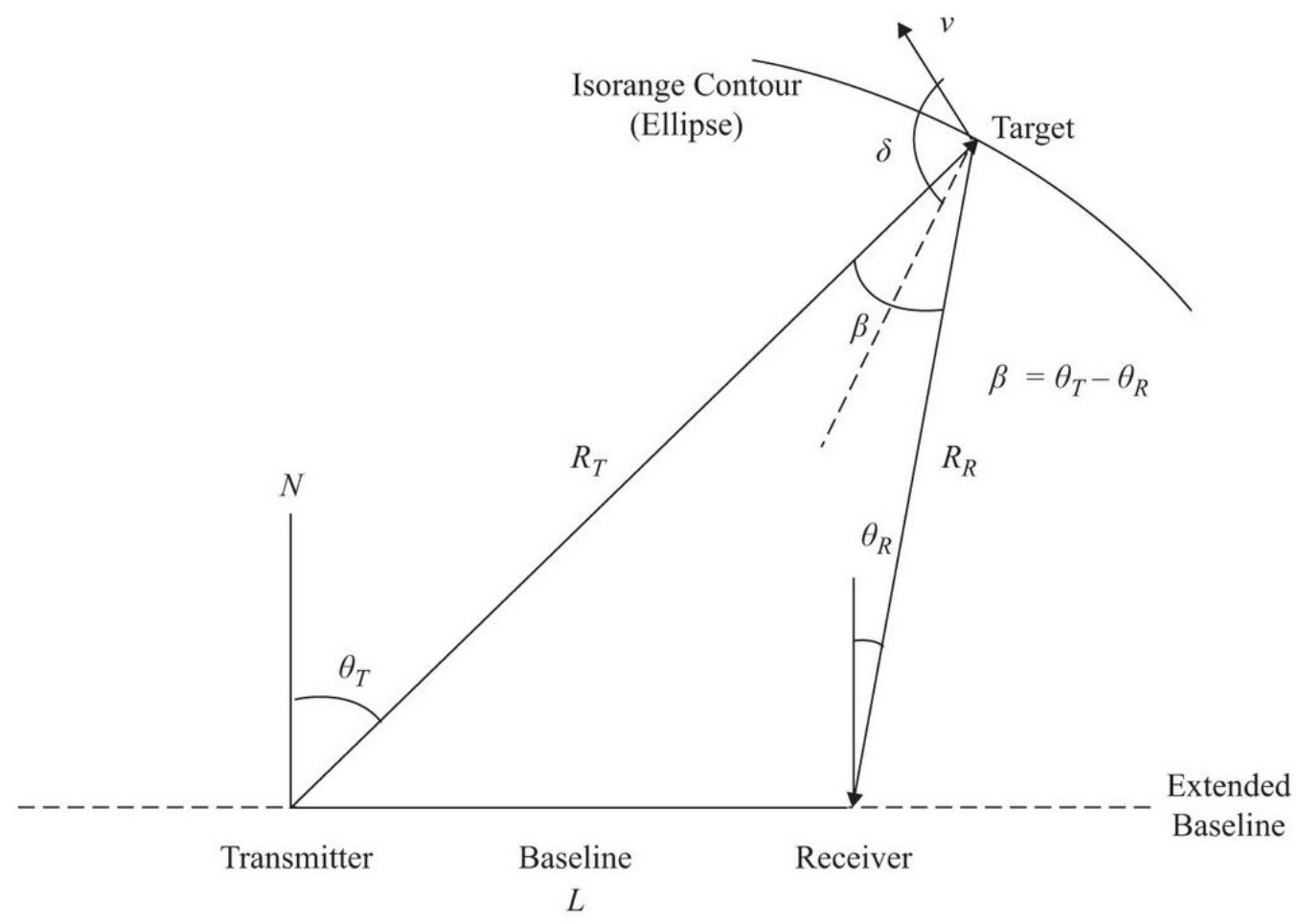

Figure 1-7: Geometry for bistatic radar setup [8]

An ellipse can be defined by contours of constant bistatic range sum $\left(R_{T}+R_{R}\right)$ with the transmitter and receiver at the two foci; this is seen in Figure 1-8. The range sum $\left(\mathrm{R}_{\mathrm{T}}+\mathrm{R}_{\mathrm{R}}\right)=0.5 * c \tau$ can be extracted from the observable quantity $\left(\mathrm{R}_{\mathrm{T}}+\mathrm{R}_{\mathrm{R}}-\mathrm{L}\right)$ once $\mathrm{L}$ is known. 


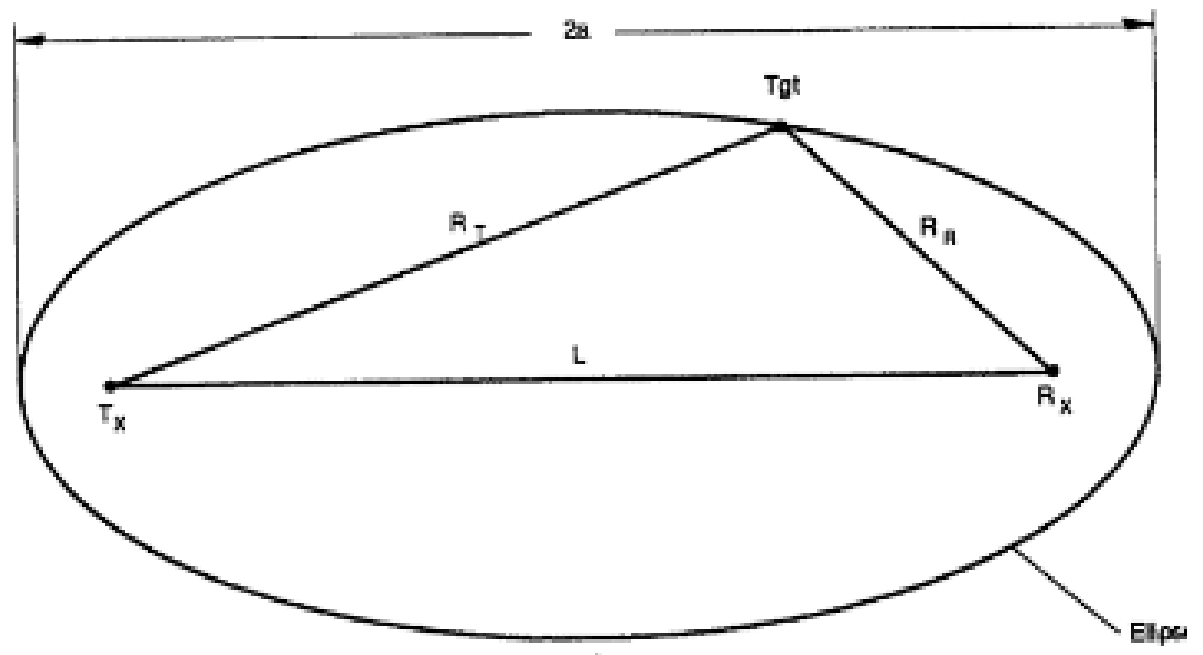

Figure 1-8 : Constant range sum ellipse with bistatic geometry [7]

Using equation 1.5, the range of the target from the receiver may be gotten if $\theta_{\mathrm{R}}$ is measured. [8]

$R_{R}=\frac{\left(R_{T}+R_{R}\right)^{2}-L^{2}}{2\left(R_{T}+R_{R}+L \sin \theta_{R}\right)}$

The Doppler shift on the echo is generally obtainable from the rate of change of the transmitter-target-receiver path when transmitter, target and receiver are all moving at different rates. When both transmitter and receiver are immobile, the Doppler shift $\mathrm{f}_{\mathrm{b}}$ on the received echo is given by equation 1.6 where $\beta$ is the bistatic angle, $v$ is the target velocity, $\mathrm{f}_{\mathrm{t}}$ is the incident frequency on the target and $\delta$ is the angle of velocity with respect to the bisector of the bistatic angle, also known as aspect angle [8].

$f_{b}=\frac{2 v f_{t}}{c} \cos \delta \cos (\beta / 2)$ 
When $\beta=0^{\circ}, \mathrm{f}_{\mathrm{b}}$ reduces to the monostatic case. But this is for a monostatic radar, which is located on the bistatic bisector with contours that are hyperbolas. The bistatic Doppler's magnitude can never be greater than that of this monostatic Doppler $f_{m}$ as per equation 1.2. When $\beta=180^{\circ}, \mathrm{f}_{\mathrm{b}}=0$ for any $\delta$, which is the forward-scatter case. The bistatic Doppler is zero when $\delta= \pm 90^{\circ}$. Because these velocity vectors are also tangent to a range-sum ellipse at this point, all such ellipses (including the baseline) become contours of zero target Dopplers. The bistatic Doppler is a maximum when $\delta=0^{\circ}$. All such hyperbolas become contours of a maximum target Doppler as this velocity vector is also tangent to a hyperbola orthogonal to the range-sum ellipse at this point [5]. The velocity vector is pointed at the transmitter or receiver when $\delta= \pm \beta / 2^{\circ}$; this sometimes is shown in the literature as a special case of equation 1.6.

The bistatic max range equation is similar to that of a monostatic equation except the range used for power transmitted and received are different. The bistatic cross section area is some factor of the mono cross section which is dependent on the shape of the target and the angle of reflection from the target relative to the incidence of the transmitted radar signal. The bistatic range equation can be represented by equation 1.7. $R_{t}^{2} R_{r}^{2}=\frac{P_{t} G_{t} G_{r} \sigma_{\text {bistatic }} \lambda^{2}}{(4 \pi)^{3} P_{r} \min }$

where the peak power transmitted is $P_{t}$, the transmit and receive gains respectively are $G_{t}$ and $G_{r}$; the bistatic target cross-section is $\sigma_{\text {bistatic }}$, signal wavelength is $\lambda$, range from transmitter to target $\mathrm{R}_{t}$, range from target to receiver $\mathrm{R}_{\mathrm{r}}$ and the minimum received power 
$P_{r}$. Hence, the maximum range $\left(\mathrm{R}_{\mathrm{t}} \mathrm{R}_{\mathrm{r}}\right)_{\max }$ is limited by the power received and the bistatic radar cross section.

The RCS, $\sigma$, in monostatic radar is a measure of the energy scattered from the target in the direction of the receiver as is the case with bistatic radar cross section of a target, $\sigma_{b}$. Because $\sigma_{b}$ is a function of the aspect angle and bistatic angle, bistatic cross sections are inherently more complex than monostatic ones. Pseudomonostatic, bistatic, and forwardscatter (occasionally referred to as near-forward-scatter) are three areas of interest of bistatic RCS. The bistatic angle defines each region, whose extent is essentially determined by the target's physical characteristics. [7]

\subsection{Comparison between Monostatic and Multistatic Radar}

Because stealth aircraft have conventionally been optimized essentially for the monostatic type of radar, as a possible counter-stealth proposal, using the multistatic approach has been suggested. It may be useful to reiterate that shaping - the basic stealth principle - is used to reflect the radar energy to irrelevant directions, not back to the emitting radar. The multistatic radar approach involves employing one or more receiver antennae positioned in such a way as to receive the scattered echo. [6]

Radar systems like these offer increased survivability; this is because the receivers are redundant and their operation passive. By the elimination of one receiver, the system may still operate despite degraded performance. [6] 
A multistatic radar can be used to improve resolution and parameter estimation, which is commonly poor in the cross-range dimension for monostatic radars. A multistatic radar can increase surveillance coverage by spreading the radar geometry. [1]

A monostatic radar requires directional antennas that rotate on a platform to locate targets as compared to a multistatic radar that uses geometric lines of position gathered from stationary non-directional antennas.

A multilateration process often involves locating an object by precisely computing the Time Difference of Arrival (TDOA). The accurate determination of a 2D target position requires a minimum of two separate TDOA measurements. While multilateration is frequently used in secondary surveillance radar, there is a real added difficulty with multistatic radar: targets are inherently non-cooperative. For example, in a multi-target scenario, received signals do not always reveal from which target they originated. Because of this missing information, there is a much greater risk for ambiguity when dealing with multiple targets. Figure 1-9 offers an example of this with the appearance of 'ghost targets'. Despite not being a real multistatic problem, the problem of multipath, or jamming through the retransmission of received signals, is similar to that of 'ghost targets'. The most obvious solution to such problems is the provision of more information, which can come from tracking over time, using Doppler information or by using further transmitter-receiver pairs. [1] 


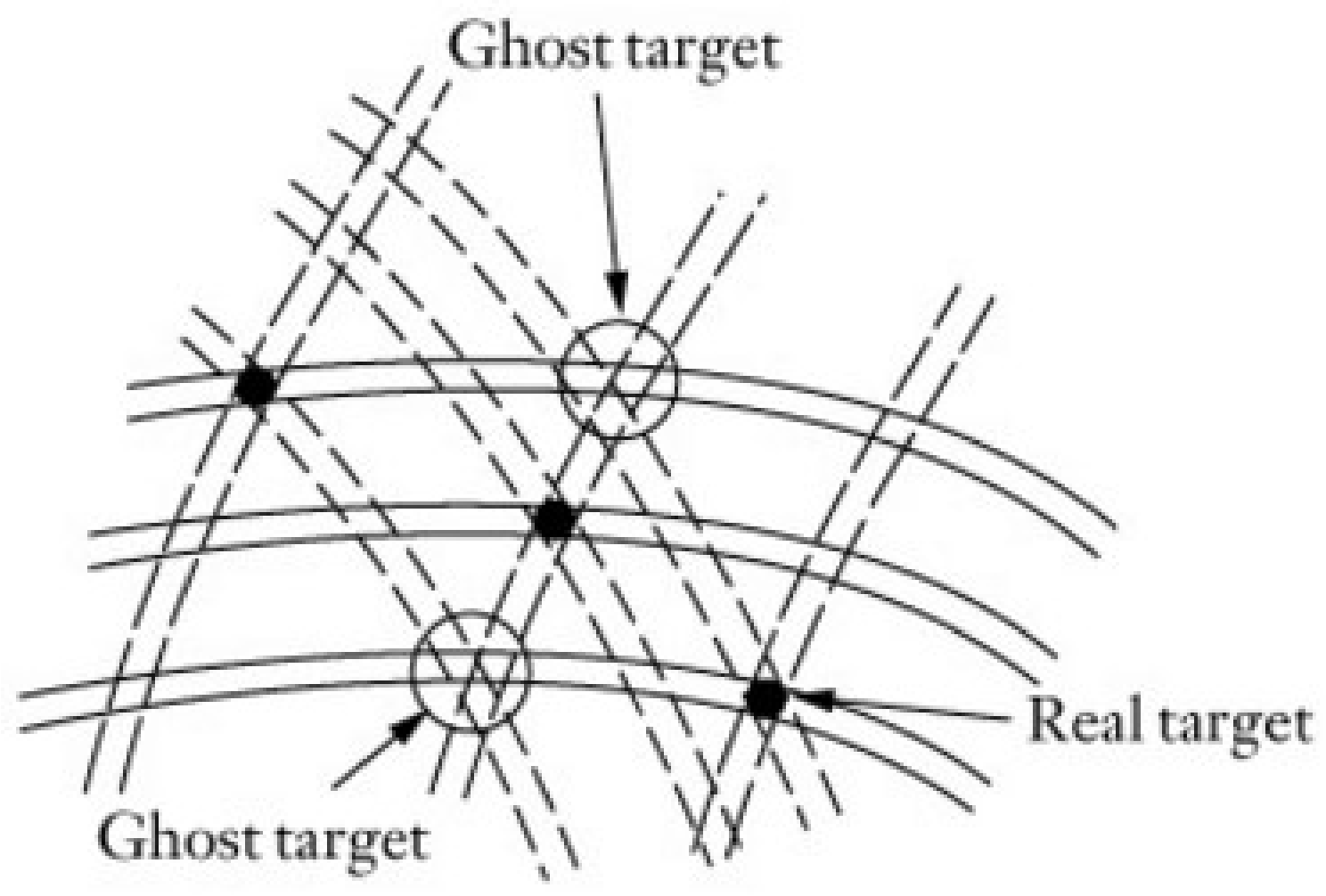

Figure 1-9: Ghost target due to multiple targets under surveillance [2]

\subsection{ISR (Intelligence Surveillance and Reconnaissance)}

"Intelligence is the product of processed information concerning hostile or potentially hostile forces. Surveillance is the systematic observation by technical sensors or human beings. This implies continuous 24 hours a day/7 days a week surveillance of areas or forces of interest. Reconnaissance is the directed mission(s) to obtain specific information. ISR is the capability that integrates command direction, sensors, and processed information and intelligence with timely dissemination in order to provide decision makers with effective 'Situational Awareness' [10].” 
Early warning radar systems are a good example of an ISR asset. Early detection/warning provided the impetus for radar development. One of the very earliest of such a system was the British Chain Home radar; by using a bistatic forward scatter setup, it was of immense importance in the success of the Battle of Britain during the Second World War. Interest in early warning radar systems has continued into the twenty-first century with many voices recognizing their vital importance to national defense. Such early warning radar systems work in tandem with command-and-control networks and also offer handoff to tracking and engagement radar [8].

A multistatic radar is used to detect stealth aircraft by the multilateration technique; this radar system will serve as an ISR asset for the military providing directional information of the target's whereabouts.

\subsection{Thesis Overview}

Chapter 2 begins with a discussion of multilateration techniques such as Time Difference of Arrival (TDOA) and Frequency Difference of Arrival (FDOA) in light of the multistatic radar setup that will be used throughout this research. Chapter 3 will review the non-linear data fusion technique to solve non-linear simultaneous equations to geolocate target positions. In Chapter 4, a filtering technique using a prediction algorithm, namely Kalman Filtering (KF), will be covered in detail with an application in the multilateration technique. Chapter 5 will present all the simulation permutation results and comparisons while demonstrating the dual stage method of gathering additional data. Finally, Chapter 6 will discuss the simulation results and conclude with recommendations on how to improve the given research. 


\section{Chapter 2 Multilateration Technique for Geolocation in 2D}

In this section, after a definition of the multilateration technique, the TDOA and FDOA equations will be derived along with how to extract this information from a multistatic radar setup. The geometry of an antenna setup relative to target location will be highlighted as well.

\subsection{What is Multilateration Technique}

Passive bistatic radars (PBRs), comprise subset of bistatic radars. For their source of radiation, they exploit non-radar transmitters of opportunity. PBRs are designed for air surveillance used in civil and military applications. If more than one transmitter is used simultaneously, the resultant configuration is a multistatic one. In this instance, measurements generated from transmit-receive pairs having overlapping coverage can be integrated for locating a target, usually by mulitlateration, by determining for example, the intersection of iso-range contours, which are generated by each pair. One transmitter, employing multiple receivers, is also a multistatic radar, which can be used for multilateration. [2]

Multilateration is the term used to denote hyberbolic lines of position based on Time Difference of Arrival (TDOA) measurements and/or elliptical lines of position based on Frequency Difference of Arrival (FDOA) measurements in computing the range of a target using multistatic radar. [2] 
In fact, multilateration systems possess two characteristics unique in the radar world: (a) these range-independent, Geometric Dilution of Precision (GDOP) driven location estimates, and (b) ghosts, or false targets that inevitably appear in a multitarget environment that can be observed in Figure 1-9 [2]. The effects of GDOP will be detailed in following chapter.

\subsection{Assumptions and Limitations}

For simplicity, we will limit all sensors/receivers and the target to one plane turning the experiment to a $2 \mathrm{D}$ space. The target is modeled as an emitter source assuming a transmitter has sent out a beam towards the target and we are now only observing the reflections off the moving target. Scattered multiple receivers pick up the signals to be processed at a Central Station (CS). To mitigate the effect of multipath hence ghost target, we will assume the radars are in the Line Of Sight (LOS) and above ground, away from the effect of multipath where the signal can bounce from nearby objects on the ground. Hence the receivers can be placed on an Unmanned Aerial Vehicle (UAV) with known location and velocity. To further simplify the experiment we will assume the receivers are stationary such that the UAV is hovering in a spot with a known location using Global Positioning System (GPS) or shared global clock such as Network Time Protocol (NTP). The velocity of the aircraft will be assumed to be steady over the duration of surveillance.

The target state consists of position $s_{t}$ and velocity $s_{t}^{\prime}$ denoted by labels in equation 2.1 .

$$
\begin{gathered}
s_{t} \\
s_{t}^{\prime}=\left(x_{t}, y_{t}\right)
\end{gathered}
$$


The sensor states consist of position data only as velocity is set to zero for all receiver sensors. The position data is represented by labels in the equation 2.2 with subscript $\mathrm{i}$ indicating the i'th sensor location.

$s_{i}=\left(x_{i}, y_{i}\right), \mathrm{i}=1,2,3 \ldots$

The distance between each receiver $i=1,2,3 \ldots$ and target pair is indicated by equation 2.3 .

$\left\|r_{i}\right\|=\left\|s_{i}-s_{t}\right\|=\sqrt{\left(x_{i}-x_{t}\right)^{2}+\left(y_{i}-y_{t}\right)^{2}}, \mathrm{i}=1,2,3 \ldots$

The difference in position from a receiver to a target is represented by vector $r_{i}$. Taking the magnitude of this vectors resulted in the distance. The notations in equation 2.1 and 2.2 along with the distance from target to the $i$ 'th receiver in equation 2.3 will be used to derive the equations for the following sections.

\subsection{Time Difference of Arrival (TDOA)}

Time Difference of Arrival (TDOA) is a locating technique whereby a target can lie on a hyperbolic Line of Position (LOP) relative to two sensors. This is done by measuring the TDOA of the signal from the target to the two respective sensors. The difference in distance between the first sensor and target to the second sensor and target is proportional to the TDOA measured between sensors one and two [9]. This is illustrated in equation 2.4 where the difference in distance is proportional to TDOA with c, speed of light being constant.

$T D O A=\frac{\left\|r_{i}\right\|-\left\|r_{j}\right\|}{c}$ where $i=1,2,3 \ldots j=1,2,3 \ldots$ and $i \neq j$ 
Along a single LOP, the difference in arrival of signal to the respective sensors from any location on the LOP remains constant, hence resulting in an infinite number of solutions. To narrow the problem a second LOP can be introduced using a third sensor. Hence the intersection between the two LOP's results in a target location in a 2-D (Dimensional) plane as can be seen in Figure 2-1.

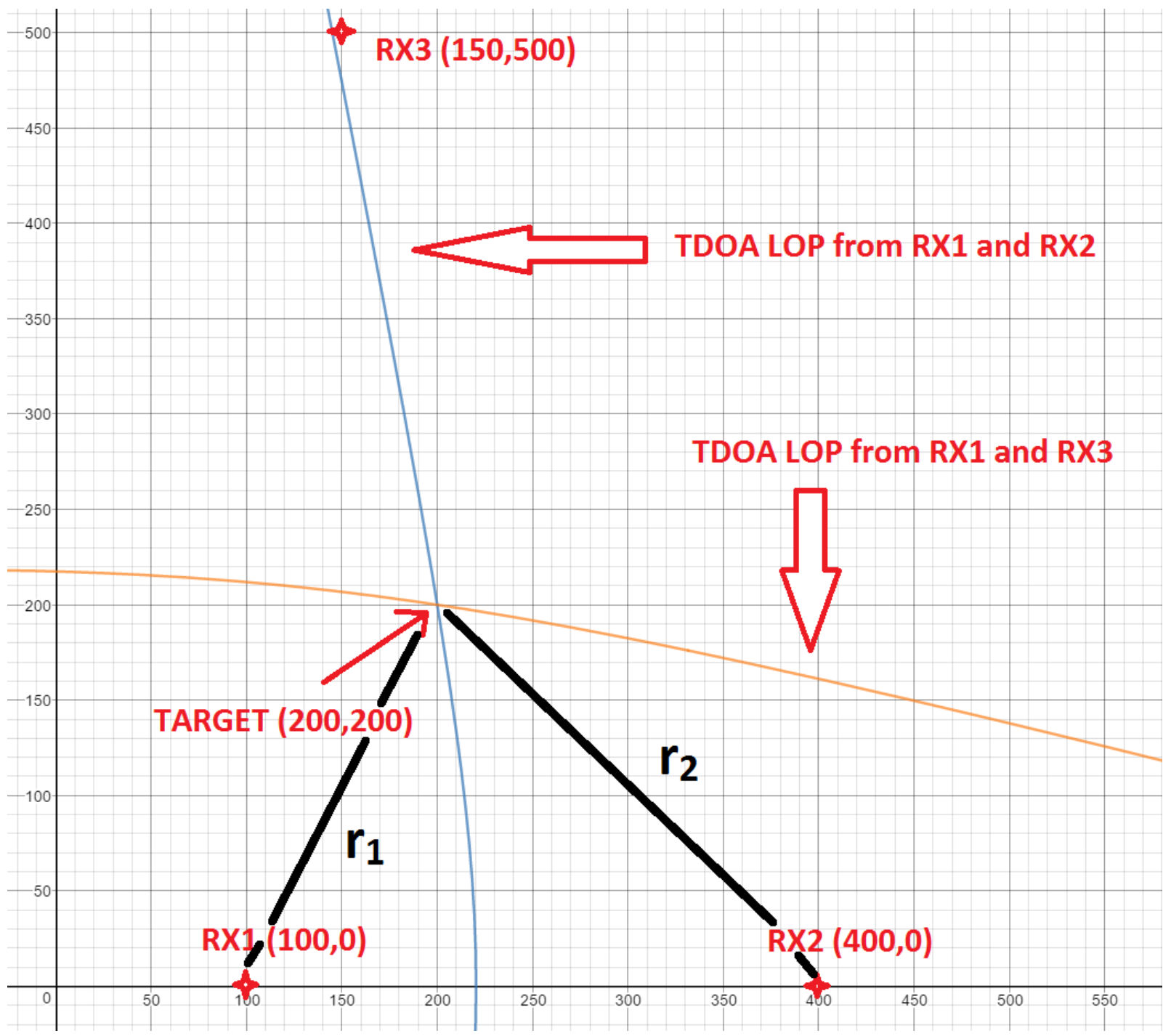

Figure 2-1 : Three receivers with 2 LOP intersecting to indicate target location using $T D O A$ 
The intersection of these hyperbolic lines brings about the problem of solving the nonlinear TDOA equations from each receiver pair. Because the solution of the TDOA equations lies on a hyperbolic line, fusing the LOP to locate the target will result in nonlinear measurements. By reducing the time between samples, one can assume linearity of the measurements. This will be an important assumption when Kalman Filter will be used to filter the noisy measurements.

\subsubsection{Derivation of TDOA}

The derivation of the hyperbolic TDOA equation will require a signal source, a target and at least two receiver sensors. To begin we will derive the TOA to the ith sensor assuming a total of $\mathrm{N}$. The Time of Arrival (TOA) is the total time it takes for the signal to travel from the transmitter to the target and finally arriving at the ith receiver. This is represented by equation 2.5 where $t_{i}$ is the TOA to the ith receiver.

$$
t_{i}=t_{0}+\frac{\left\|r_{i}\right\|}{c}+\varepsilon_{i}, \mathrm{i}=1,2,3 \ldots \mathrm{N}
$$

The TOA is composed of $t_{0}$ indicating the time stamp when the signal was transmitted, magnitude of vector $r_{i}$ indicating the distance from the target to the ith receiver and $\varepsilon_{i}$ indicating the error in time arrival measurement. To determine the TDOA, we need a second TOA measurement such that we get $\mathrm{t}_{\mathrm{i}+1}$ or the ith plus one measurement. Therefore, by taking the difference between the two TOA, we have equation 2.6 representing TDOA.

$T D O A_{i}=t_{i+1}-t_{i}=\frac{|| r_{i+1}||-|| r_{i}||}{c}+n_{i}, \mathrm{i}=1,2,3 \ldots \mathrm{N}-1$ 
The TDOA equation represents a hyperbolic curve where the difference in distance is constant and the time is measured by using the speed of light $\mathrm{c}$. The noise $\mathrm{n}_{\mathrm{i}}$ is the difference in error between the TOA error $\varepsilon_{\mathrm{i}+1}$ and $\varepsilon_{\mathrm{i}}$. The total number of TDOA measurements is $\mathrm{N}-1$ compared to the total number of receivers $\mathrm{N}$.

\subsubsection{Extract TDOA Measurement}

There are several important factors to consider before extracting the TDOA. First, the BW of the incoming signal should be large as the time resolution is inversely proportional to the signal BW. The signals on both sensors need to be time aligned either with an atomic clock or GPS. Signals should not be continuous wave but rather pulse form such that there is a definite start of signal that can be identified, then taking the difference in TOA will result in the TDOA. The Doppler on the signal should be removed before extracting the TDOA using autocorrelation. In order to do this one has to know the frequency at which the signal of opportunity is operating at. Autocorrelation can be used on continuous wave signals under certain conditions to identify difference in TOA [13].

It can see from Figure 2-2 that signal 1 is a pulse signal with the frequency $20 \mathrm{~Hz}$. It is 200 time units and so the BW would be the inverse of the time unit. If, for example, by taking the time unit to be a microsecond then the BW would be $5 \mathrm{kHz}$. 


\section{Signal 1}

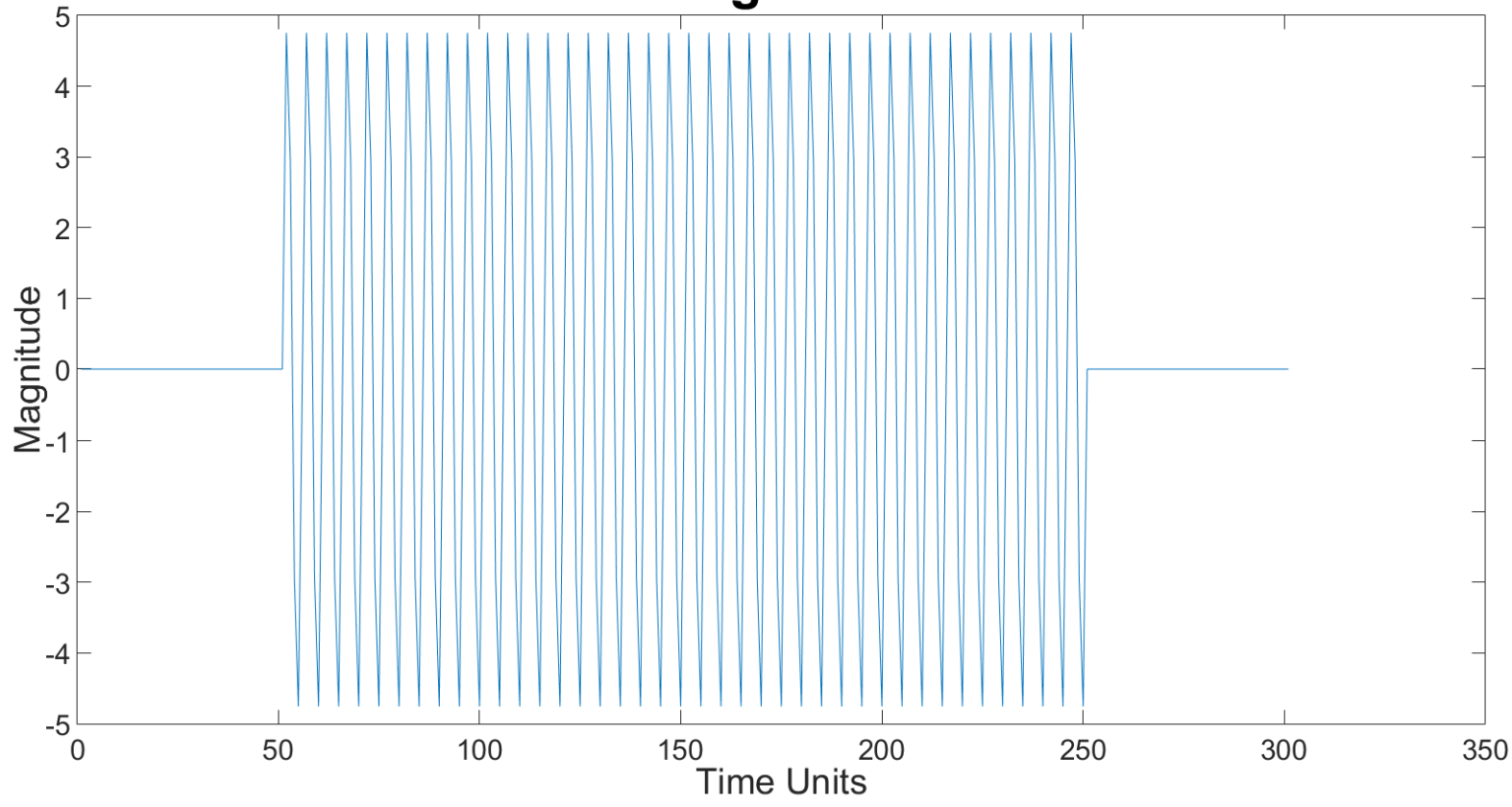

Figure 2-2 : Signal 1 with pulse 200 time units and freq. $20 \mathrm{~Hz}$ starting at 50 time units

Figure 2-3 shows signal 2 arriving at a separate receiver from a target with the frequency 10Hz. The signal starts at time unit 100. It can immediately seen that the time difference is 50 time units when comparing signal 1 in Figure 2-2 to signal 2 in Figure 2-3. 


\section{Signal 2}

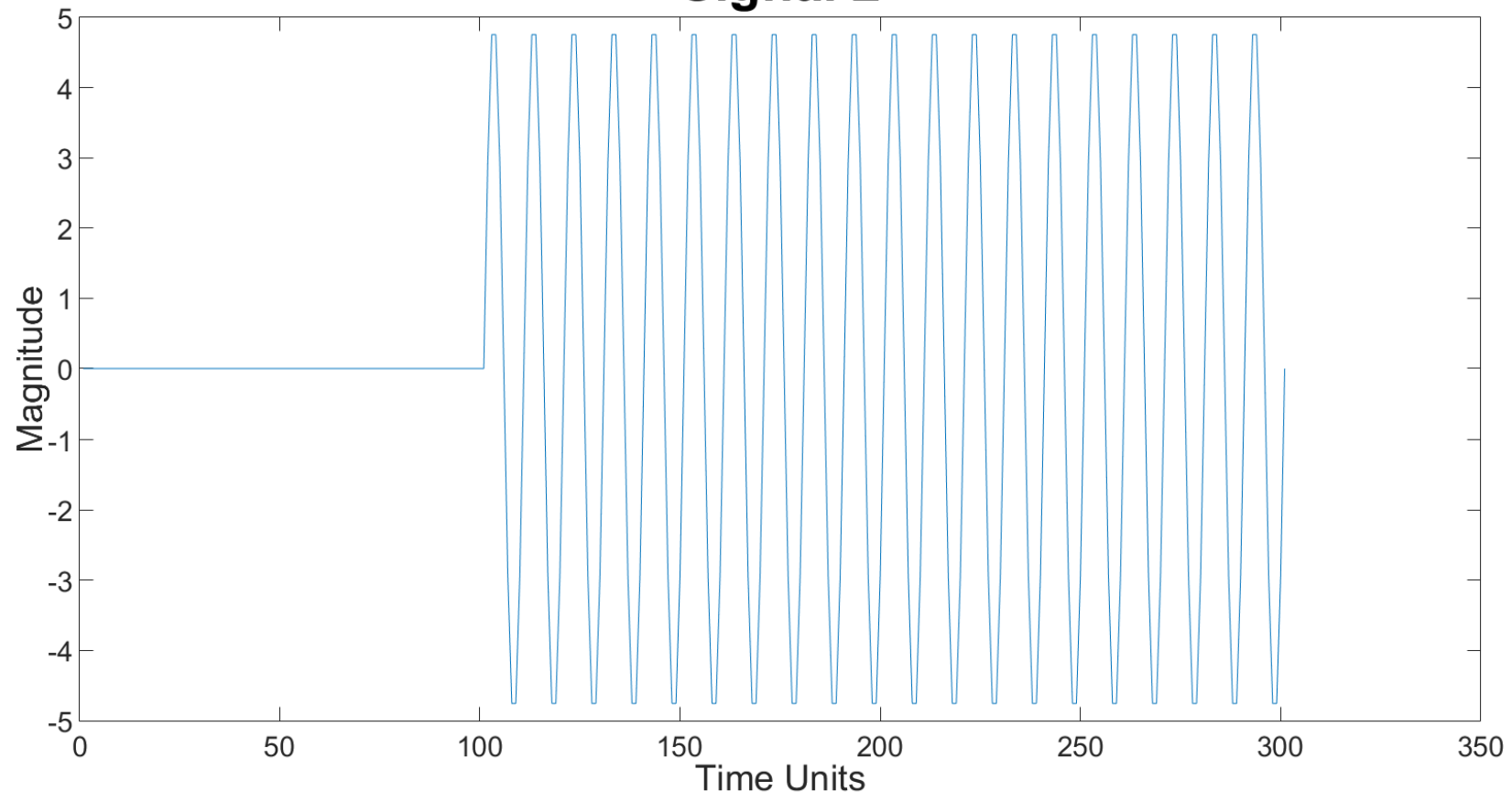

Figure 2-3 : Signal 2 with pulse 200 time units and freq. $10 \mathrm{~Hz}$ starting at 100 time units

To determine the TDOA the use of cross correlation between signal 1 and signal 2 , the signal $\mathrm{k}(\mathrm{t})$ coming from the target to the receivers $l_{1}(\mathrm{t})$ and $\mathrm{l}_{2}(\mathrm{t})$ as signals 1 and 2 respectively seen in equation 2.6.

$l_{i}(t)=A_{i} * k\left(t-t_{i}\right) e^{-j w_{i} t}+n_{i}(t)$, where $\mathrm{i}=1,2,3 \ldots$

The $t_{i}$ represents TOA to receiver $l_{i}$ with amplitude $A_{i}$ and frequency $\mathrm{w}_{i}$ that is $2 * \Pi^{*} f_{i}$ with noise $\mathrm{n}_{\mathrm{i}}$ that is stationary random process. That means the signal is not correlated with the noise. Going ahead with the cross correlation the phase shifts due to a difference in frequency; a distorted result thus is seen in Figure 2-4. 


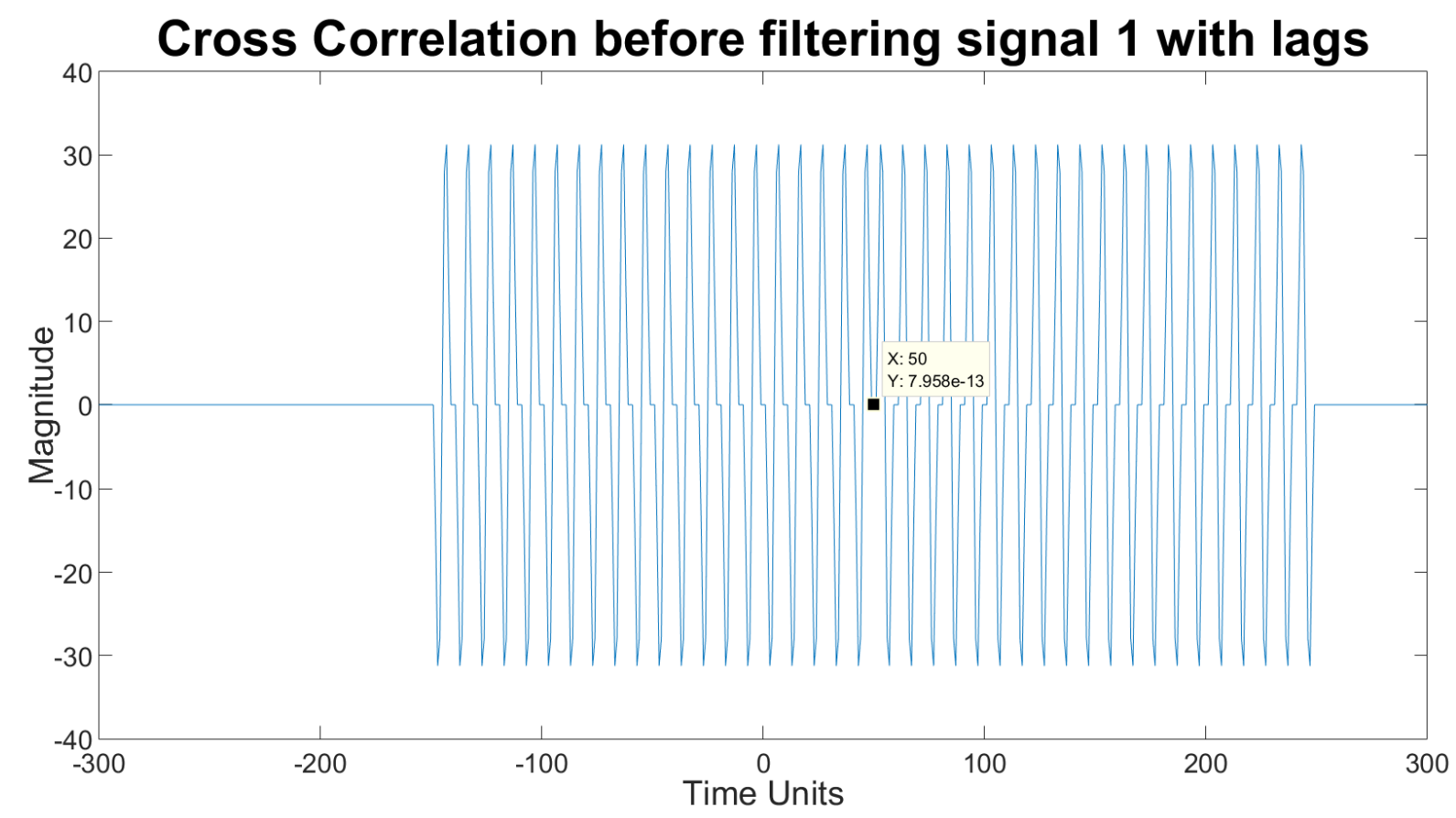

Figure 2-4 : Cross correlation before filtering signal 1

If the Doppler between signals 1 and 2 are removed, then it will have a better correlation result. To do this it will need to down convert one of the signals and filter out the higher frequency components. From section 2.4.2 it can see the frequency difference between signals 1 and 2 is $10 \mathrm{~Hz}$, so down shifting signal 1 by $f_{\text {down }}=10 \mathrm{~Hz}$ using $\cos \left(2 * \Pi * f_{\text {down }} * t\right)$ the spectrum is seen in Figure 2-5. 


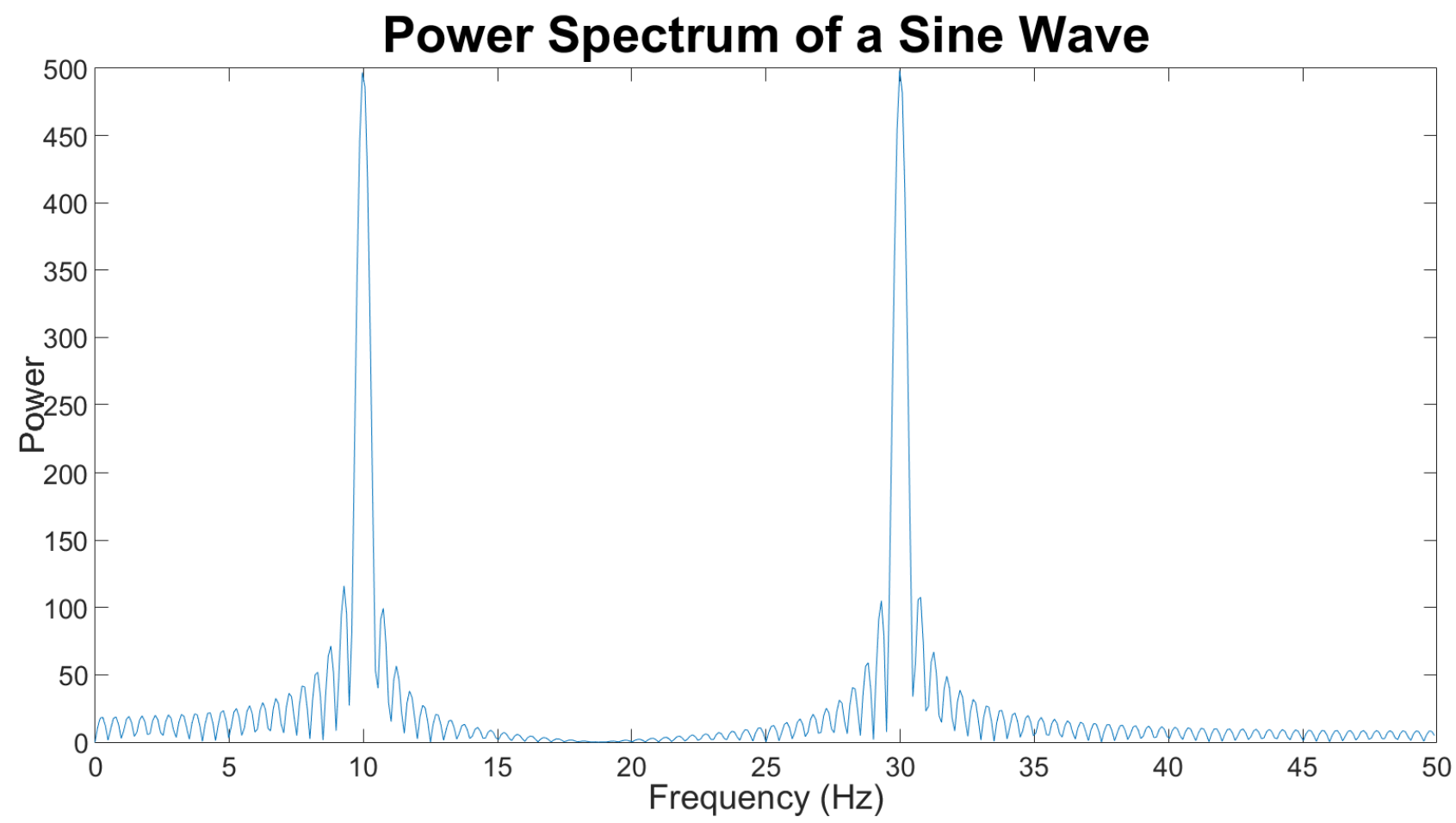

Figure 2-5 : Frequency spectrum after down converting

Using a Low Pass Filter (LPF) with cut off frequency set at $15 \mathrm{~Hz}$, we are able to discard the $30 \mathrm{~Hz}$ frequency as seen in Figure 2-5. The LPF has a response that allows lower frequencies to pass through and blocking higher frequencies past the cut off. This is illustrated in Figure 2-6 with the normalized frequency 1 being $30 \mathrm{~Hz}$, hence the $3 \mathrm{~dB}$ point is at approximately $10 \mathrm{~Hz}$. 

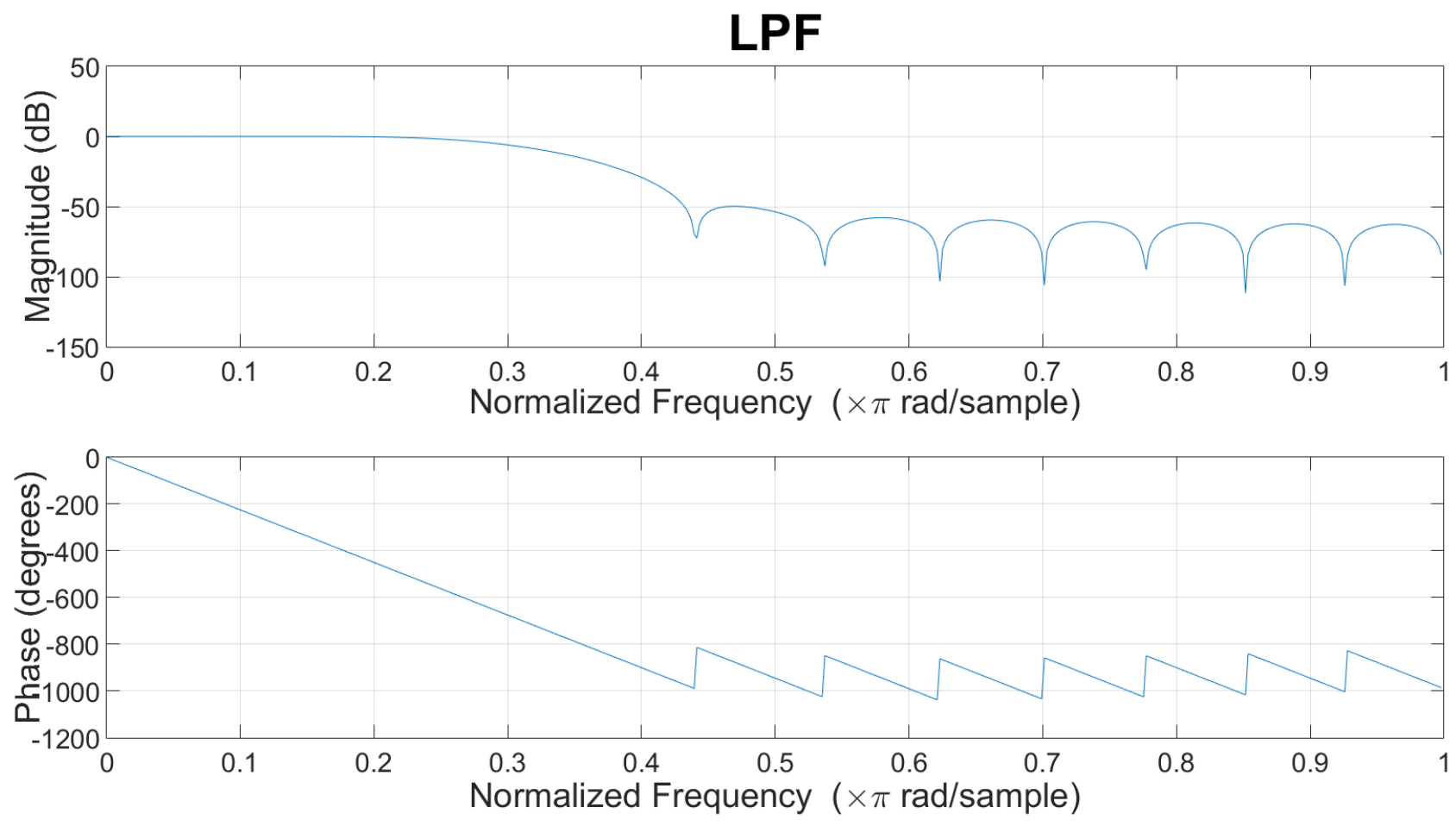

Figure 2-6 : LPF with cut off freq. $15 \mathrm{~Hz}$

From Figure 2-7, it can see that signal 1 has been filtered out by comparing it to Figure 2-

3. There is one more thing to note and that is the delay of approximately 13 time units due to the Finite Impulse Response (FIR) Low Pass Filter (LPF) with N=25 (the number of taps of the filter). Thus the delay to the output signal is approximately $\mathrm{N} / 2$. This has to be noted into the final TDOA measurement. 


\section{Signal 1 after LPF at $15 \mathrm{~Hz}$}

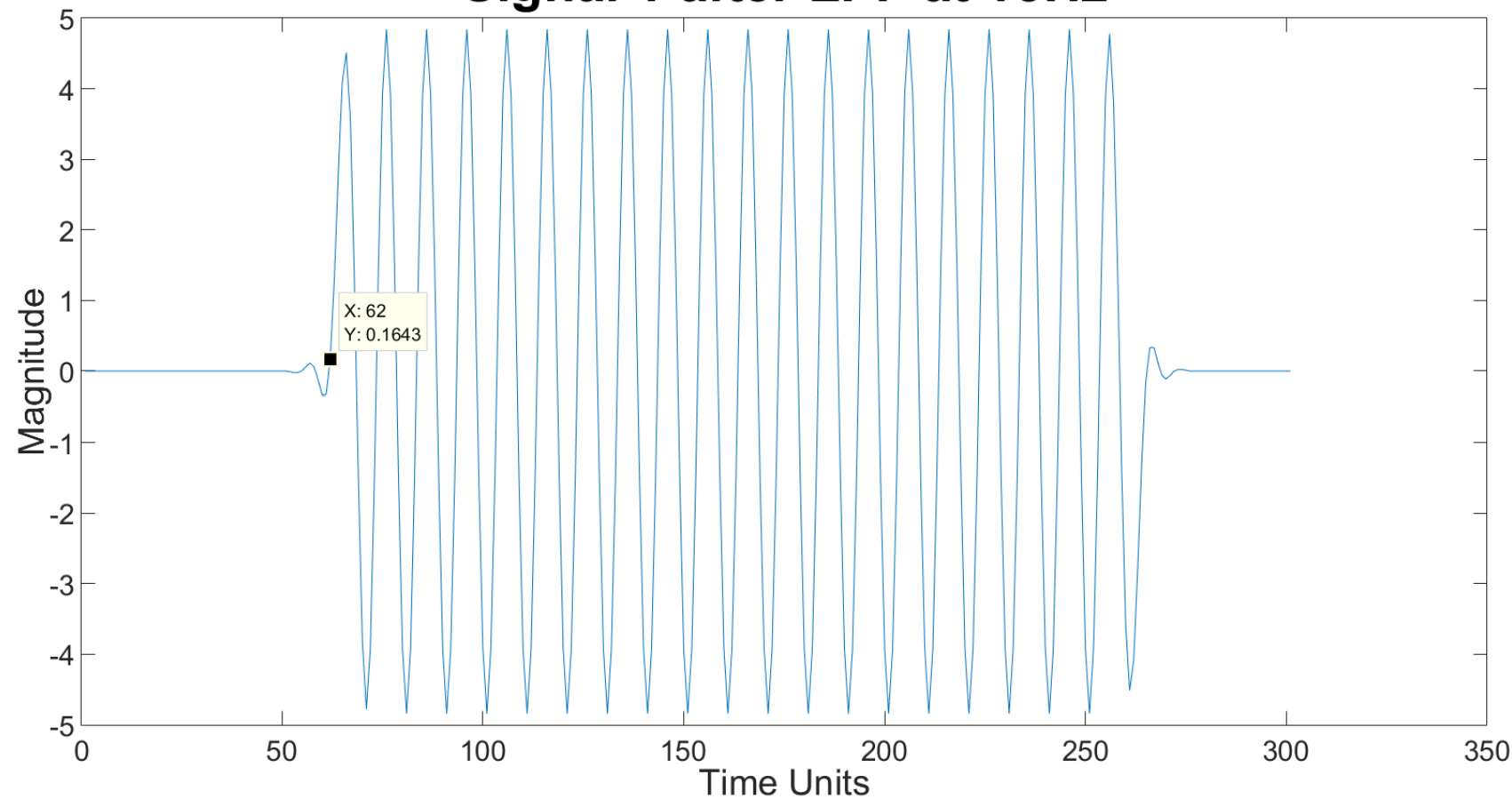

Figure 2-7 : Signal 1 after having it down shifted and filtered with LPF

Now by cross correlating signals 1 and 2 where using equation 2.7 , there is no frequency difference between signals 1 and 2 , thus the frequency $\mathrm{w}_{\mathrm{i}}$ remain the same for both and represented by w.

$l_{i}(t)=A_{i} * k\left(t-t_{i}\right) e^{-j w t}+n_{i}(t)$, where $i=1,2,3 \ldots$

The cross correlation is represented by taking the expected value of signal $l_{1}$ and $l_{2}$ with TDOA $=t_{i+1}-t_{i}$. This is seen in equation 2.8 .

$R(T D O A)=E\left[l_{1}(t) * l_{2}(t-T D O A)\right]$

Equation 2.8 can also be represented by an infinite integral as can be seen in equation 2.9.

$R(T D O A)=\int_{-\infty}^{\infty} l_{1}(t) * l_{2}(t-T D O A) d t$ 
Knowing that computing an infinite observation space is not plausible and so limiting it to an observation interval of $\mathrm{T}$ results in equation 2.10. In my experiment $\mathrm{I}$ have the observation interval of 300 time units as can be seen from Figure 2-7.

$R(T D O A)=\frac{1}{2 * T} \int_{-T}^{T} l_{1}(t) * l_{2}(t-T D O A) d t$

The cross correlation between signal 1 after being down converted to match the frequency of signal 2 gives us Figure 2-8 with a peak at 37.

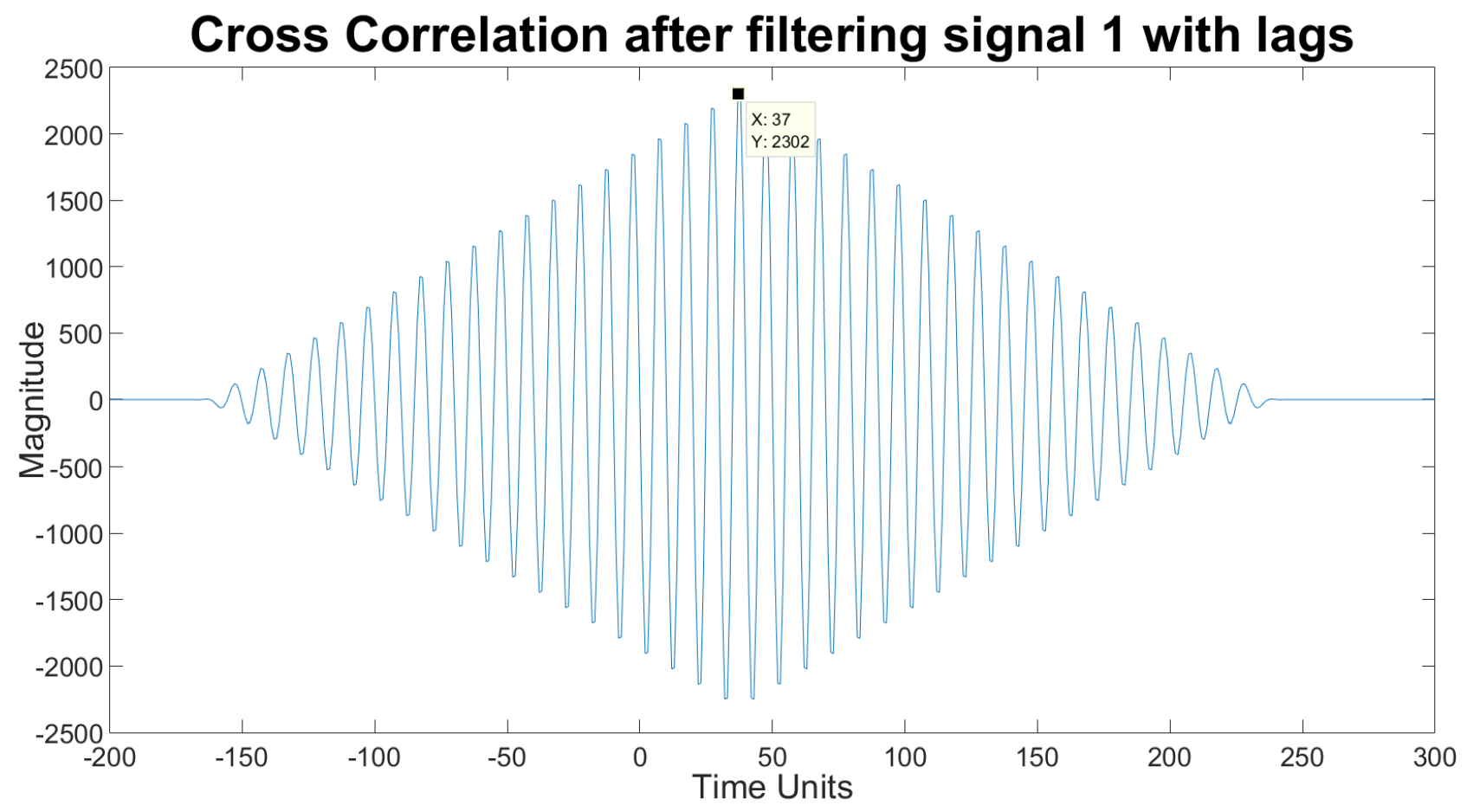

Figure 2-8: Cross correlation between signal 1 and 2 with similar frequency

The observation interval spans from -300 to 300 and that the peak occurs at 37 . Knowing the TDOA is not 37 time units but rather 50 time units. Because of the delay of 13 time 
units on signal 1, it has shortened the time difference by 13 units. To compensate, the delay is added to get an overall TDOA of 50 time units. This is due to FIR filter taps.

\subsection{Frequency Difference of Arrival (FDOA)}

Frequency Difference of Arrival (FDOA) is a locating technique whereby a target can lie on an iso-Doppler or elliptical Line of Position (LOP) relative to two sensors. This is done by measuring the FDOA of the signal from the target to the two respective sensors. With the velocity of the target and angle of reflection being different to both receivers, we get different Doppler measurements to each individual receiver. Taking the frequency of arrival measurement using equation 1.6 we get equation 2.11 with the Doppler frequency measured at $i$ 'th receiver with a total of $\mathrm{N}$.

$f_{b_{-} i}=\frac{2 v f_{t}}{c} \cos \delta_{i} \cos \left(\beta_{i} / 2\right)+\varepsilon_{i}, \mathrm{i}=1,2,3 \ldots \mathrm{N}$

It can be seen that the velocity of the target $v$ and carrier frequency $f_{t}$ remains unchanged but the aspect angle $\delta_{\mathrm{i}}$ and bistatic angle $\beta_{\mathrm{i}}$ can change depending upon receiver location relative to the target. The measurement error is represented by $\varepsilon_{\mathrm{i}}$ for each receiver. From Figure 2-9 we can see that different FDOA give rise to different iso-Doppler LOP with the target that can lie anywhere along a given LOP. The velocity vector from the target to each receiver give rise to different frequency measurements. 


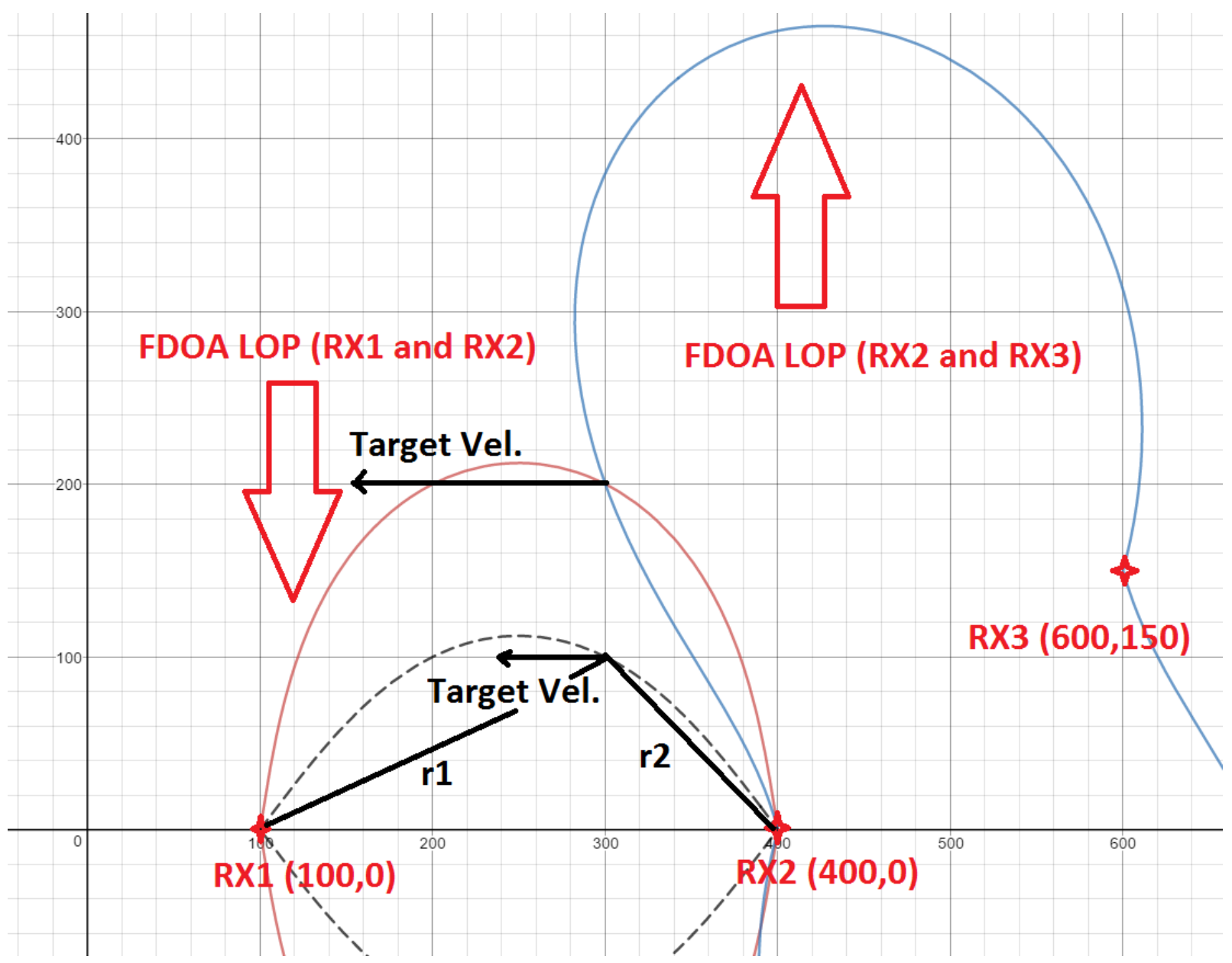

Figure 2-9: Three receivers with 2 LOP intersecting to indicate target location using FDOA with velocity vector indicated

The LOP due to Rx1 and Rx2 intersect with LOP due to Rx2 and Rx3 at location $(300,200)$ where the target lies. In Figure 2-9, the dotted LOP indicates an alternative isoDoppler LOP from Rx 1 and $\mathrm{Rx} 2$ due to the new target position $(300,100)$ with velocity the vector unchanged.

\subsubsection{Derivation of FDOA}

To derive the FDOA equation, we need the frequency Doppler at each receiver which we have already covered represented by equation 2.11. The term with the aspect angle $\delta_{\mathrm{i}}$ and 
bistatic angle $\beta_{\mathrm{i}}$ is essentially the velocity vector from the target to the ${ }^{\prime}$ 'th receiver. In other words, it is the vector $r_{1}$ and $r_{2}$ from the target to each receiver represented in Figure 2-9 for the iso-Doppler LOP with dashed lines. To represent the unit vector from the target to each receiver, we can compute the vector and divide by the magnitude that is represented in equation $2.3\left\|\mathrm{r}_{\mathrm{i}}\right\|$ and so we get equation 2.12 giving the unit vector $\mathrm{u}_{\mathrm{i}}$ from any receiver $\mathrm{s}_{\mathrm{i}}$ to the target location $\mathrm{s}_{\mathrm{t}}$.

$u_{i}=\frac{r_{i}}{\left\|r_{i}\right\|}=\frac{s_{i}-s_{t}}{\left\|s_{i}-s_{t}\right\|}=\frac{\left(x_{i}-x_{t}\right)+\left(y_{i}-y_{t}\right)}{\sqrt{\left(x_{i}-x_{t}\right)^{2}+\left(y_{i}-y_{t}\right)^{2}}}, \mathrm{i}=1,2,3 \ldots \mathrm{N}$

Thus, now representing the Doppler frequency at each receiver with equation 2.13 where the target velocity is $s_{t}^{\prime}$.

$f_{b_{-} i}=\frac{f_{t}}{c} \frac{v_{t x}\left(x_{i}-x_{t}\right)+v_{t y}\left(y_{i}-y_{t}\right)}{\sqrt{\left(x_{i}-x_{t}\right)^{2}+\left(y_{i}-y_{t}\right)^{2}}}+\varepsilon_{i}=\frac{f_{\mathrm{t}} * s_{t}^{\prime}}{c} u_{\mathrm{i}}+\varepsilon_{i}, \mathrm{i}=1,2,3 \ldots \mathrm{N}$

Taking the difference in Doppler between the two receivers we have equation 2.14 with the FDOA.

$F D O A_{i}=f_{b_{i}+1}-f_{b_{i}}=\frac{f_{\mathrm{t}} * s_{t}^{\prime}}{c}\left(u_{\mathrm{i}+1}-u_{\mathrm{i}}\right)+n_{i}, \mathrm{i}=1,2,3 \ldots \mathrm{N}-1$

The FDOA equation represents an iso-Doppler curve where the Doppler difference is constant along the LOP. The noise $n_{i}$ is the difference in error between the Doppler frequency error $\varepsilon_{i+1}$ and $\varepsilon_{\mathrm{i}}$. The total number of FDOA measurements is $\mathrm{N}-1$ compared to the total number of receivers $\mathrm{N}$. The carrier frequency $\mathrm{f}_{\mathrm{t}}$ is assumed to be given as the source of the signal is known. The target velocity $s_{t}^{\prime}$ is needed to determine the target 
position in 2D. Will discuss how the target velocity is computed using TDOA measurements and Kalman Filtering (KF) in chapter 5.

\subsubsection{Extract FDOA Measurement}

There are several important factors to consider before extracting the FDOA. First, the time integral should be as large as possible to get a sharp tall peak in the frequency domain hence improving resolution. This means receiving a signal represented by a single frequency. The signals on both sensors need to be identified so as to correlate frequencies measured coming from the same source. Fast Fourier Transform (FFT) with peak detection or cross correlation can be used to compute the frequency difference. [16]

From Figure 2-10 and Figure 2-11, the FFT of signal 1 and signal 2 has peaks occurring at $20.02 \mathrm{~Hz}$ and $9.961 \mathrm{~Hz}$ respectively. 


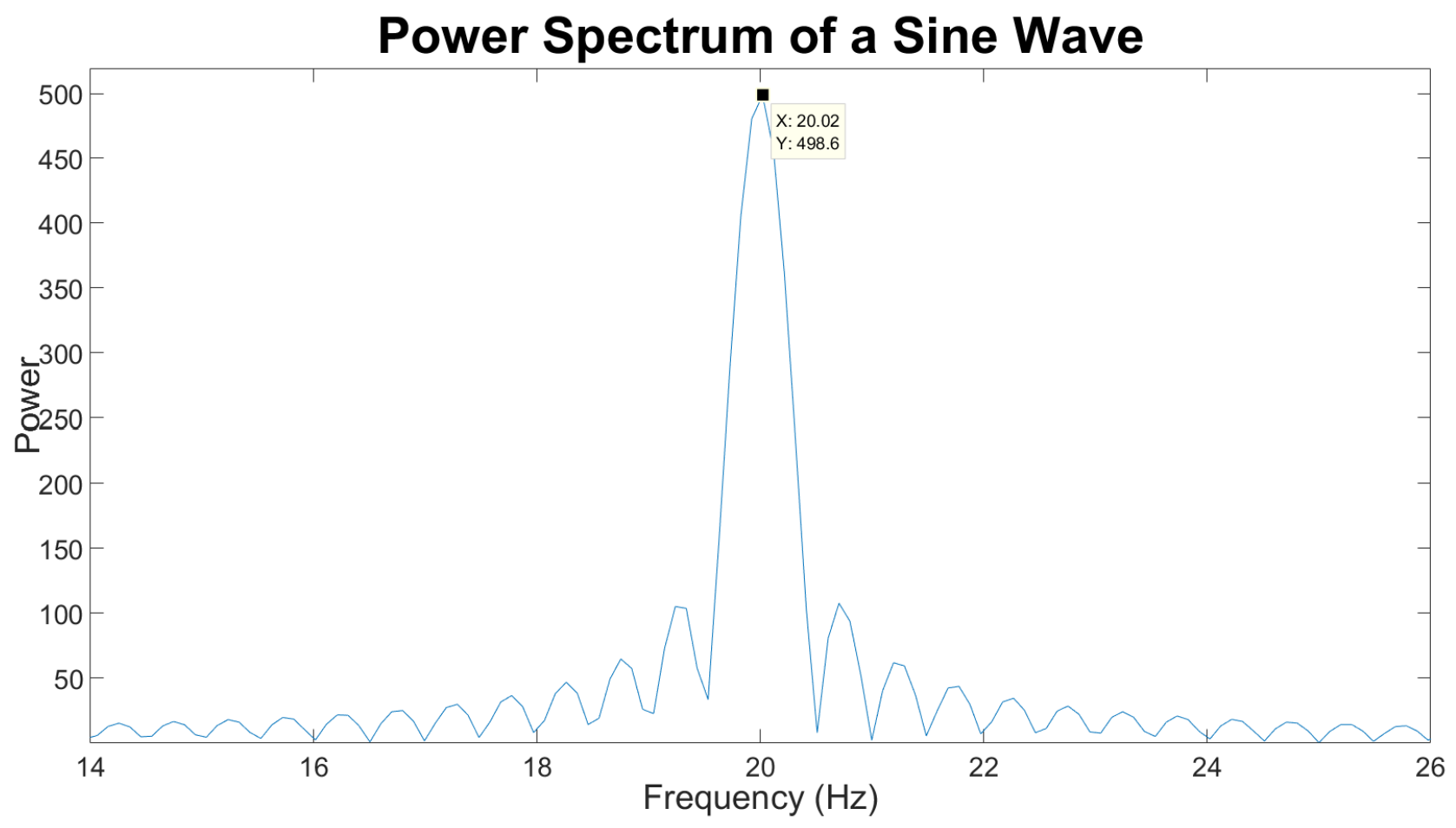

Figure 2-10 : FFT of signal 1 at $20 \mathrm{~Hz}$

It can be seen that the difference in frequency between signal 1 in Figure 2-10 and signal 2 in Figure $2-11$ is $10.06 \mathrm{~Hz}$, hence the FDOA is $10 \mathrm{~Hz}$. 


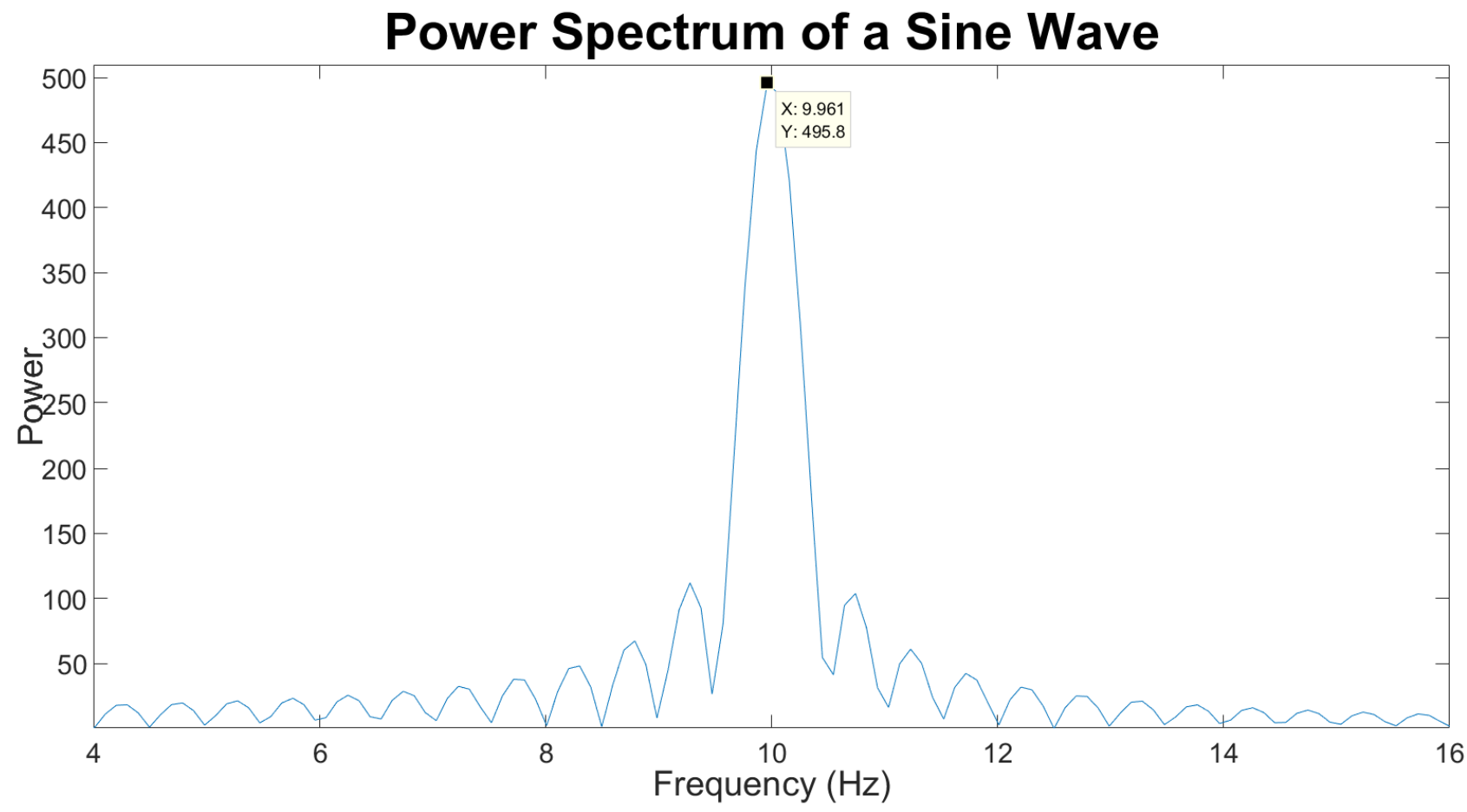

Figure 2-11: FFT of signal 2 and $10 \mathrm{~Hz}$

The FFT is essentially a representation of all possible sinusoidal waves $A_{n} e^{-j w t}$ with discrete frequencies $\mathrm{W}_{\mathrm{n}}=2 * \Pi *(\mathrm{n} / \mathrm{L})$. The FFT equation is represented by equation 2.15 .

$F_{m}=\sum_{n=1}^{L-1} f_{n} e^{-j 2 \Pi m(n / L)}$

The FFT represents discrete amplitudes $f_{n}$ for each sinusoid $n=1$ to L-1 at different discrete frequencies $\mathrm{m}$.

\subsection{Combination of TDOA and FDOA}

The combination of TDOA and FDOA generate LOPs that are orthogonal in the presence of good Geometric Dilution of Precision (GDOP). With the fusion of TDOA and FDOA measurements, only two receivers or sensors are needed in locating a target in 2-D. In 
Chapter 3, we will discuss a data fusion technique to solve non-linear TDOA and FDOA equations to locate the position of the target. Figure 2-12 demonstrates LOP due to TDOA and FDOA that intersect $(\mathrm{x}=230, \mathrm{y}=200)$ orthogonally when GDOP is good to result in more accurate location measurements.

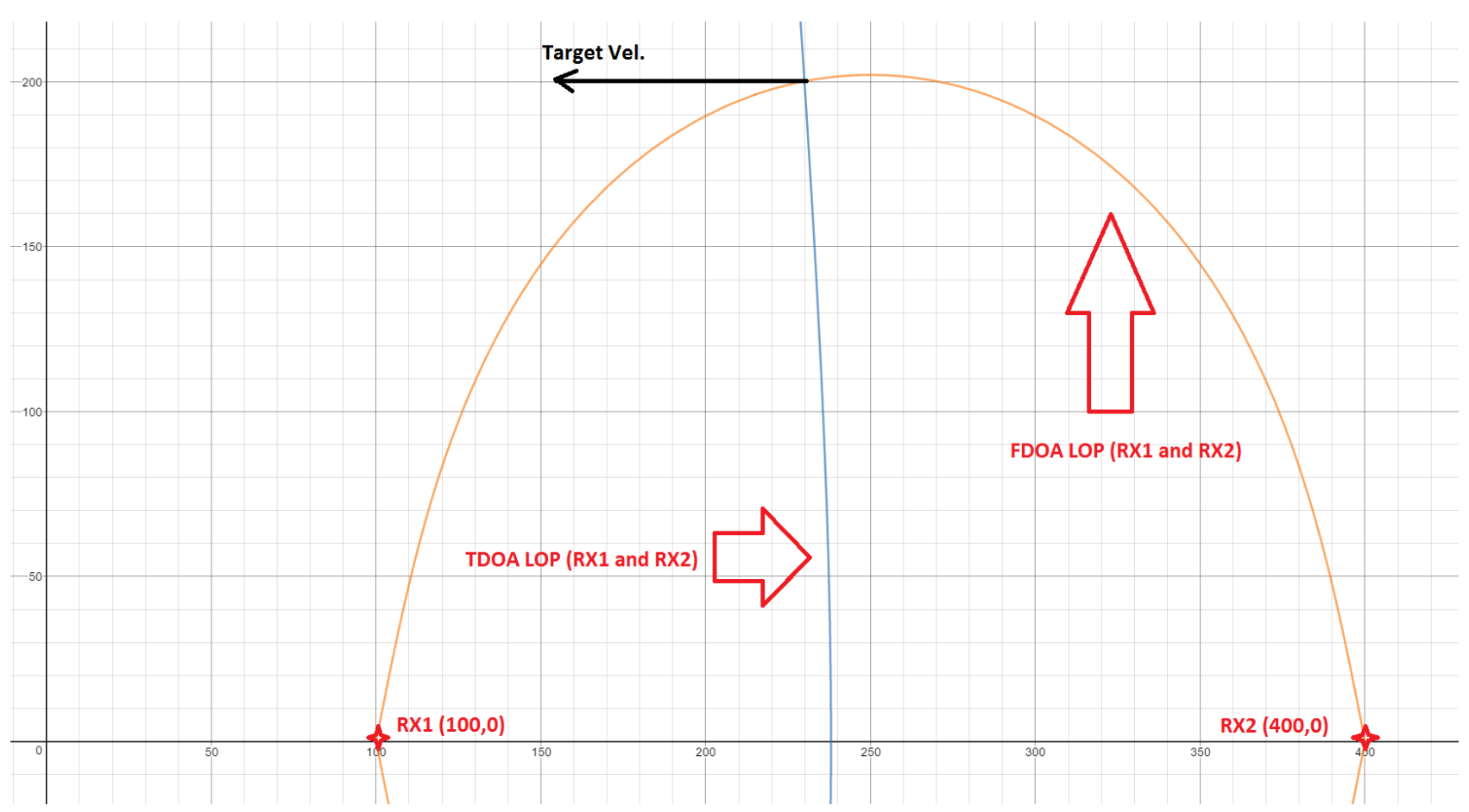

Figure 2-12: Two receivers with $L O P$ due to TDOA and FDOA

In Chapter 5 we will be using a third receiver to get a second LOP due to TDOA measurement in order to compute an estimate of target velocity. Then only two receivers will be sufficient to geolocate the target using FDOA that is velocity dependent and TDOA.

\subsection{Chapter Summary}

This chapter covered an explanation of two multilateration techniques, namely TDOA and FDOA. The the derivation of the TDOA and FDOA equations with methods to 
extract these values from our multistatic radar were covered. The LOP due to each multilateration technique was presented, and finally demonstrating a combination of both only requiring two receiver antennas. 


\section{Chapter 3 Non-linear Data Fusion Technique}

The modified Hough Transform will be used to solve non-linear TDOA and FDOA equations and fuse them to geolocate the target position. However, the target position measured will be noisy. To mitigate this noise, in the next chapter a filtering technique will help in reducing the Root Mean Squared Error (RMSE).

\subsection{Hough Transform}

The Hough Transform is used in imaging to decipher features such as lines and shapes within. It does this by clustering and fusing data to generate peaks or weights by a voting technique that results in line or shape extraction patterns. The use of the Hough Transform is beyond just image processing and has been used in multi sensor data fusion, single and multi-target tracking, localizing robot within a map and more. The original Hough Transform extracted lines only [18]. Then the Generalized Hough Transform was introduced to detect any object shapes. Finally, the Randomized Hough Transform that is more of a probabilistic variant of the Hough Transform was introduced where the properties of shapes are used to reduce computational intensity. For this thesis, I will focus on the Hough Transform and describe how a variation of it can be used for geolocation.

The Hough Transform carries out feature extraction by voting in what is called the

Parameter Space (P.S.) that creates local maxima that are accumulated in an array of $\mathrm{N}$ 
dimension, where $\mathrm{N}$ depends on the feature being extracted. For example, in line extraction the unknowns are $\mathrm{m}$ (gradient) and $\mathrm{b}$ (y-intercept) whereas for a circle it is $\mathrm{x}_{0}$ (origin of circle), $\mathrm{y}_{0}$ (origin of circle) and $\mathrm{r}$ (radius of circle).

The Hough Transform was patented by founder Paul V.C. Hough in 1962 where he describes a feature such as a line that can be extracted by taking sample points in the Feature Space (F.S.) as seen in Figure 3-1, and mapping each sample to lines in the P.S. as seen in Figure 3-2. [6] The point at which all the lines intersect in the P.S. creates a peak or higher weight. The location of the peak describes the feature by providing the gradient and y-intercept. This in return mathematically represents a line in the F.S. that best fits all samples taken to describe them in the P.S. to begin with. From Figure 3-1, we have a line with a gradient 2 and y-intercept 1 along with 5 sample points $(1,3),(3,7)$, $(5,11),(7,15)$ and $(9,19)$. These sample points are represented in the P.S. as individual lines intersecting at a common point that gives the gradient (1.99) and y-intercept (1.00) of a line that best represents the 5 sample points taken from the F.S. in Figure 3-1. 


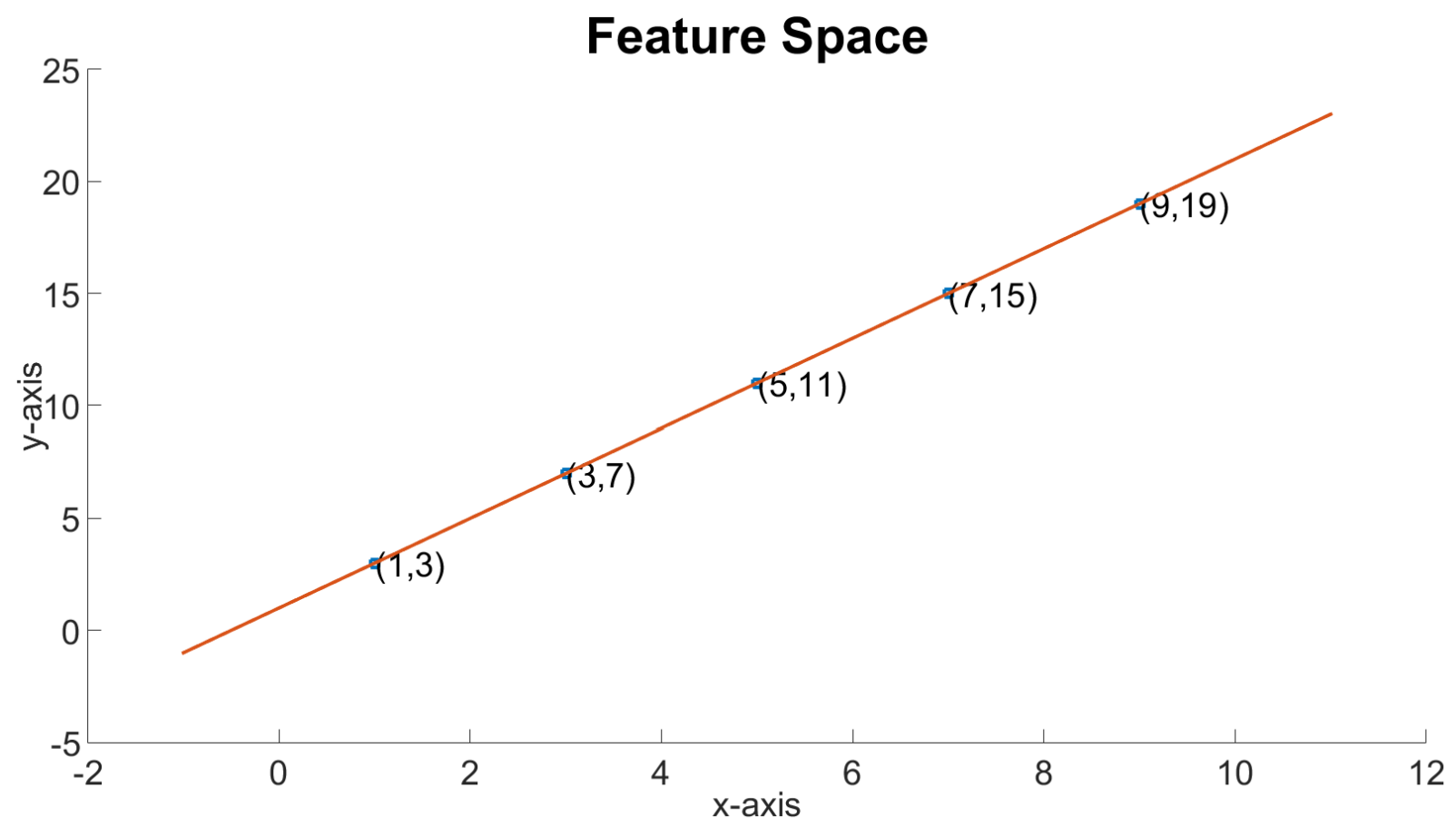

Figure 3-1 : Feature Space with 5 points used as samples for the Parameter Space

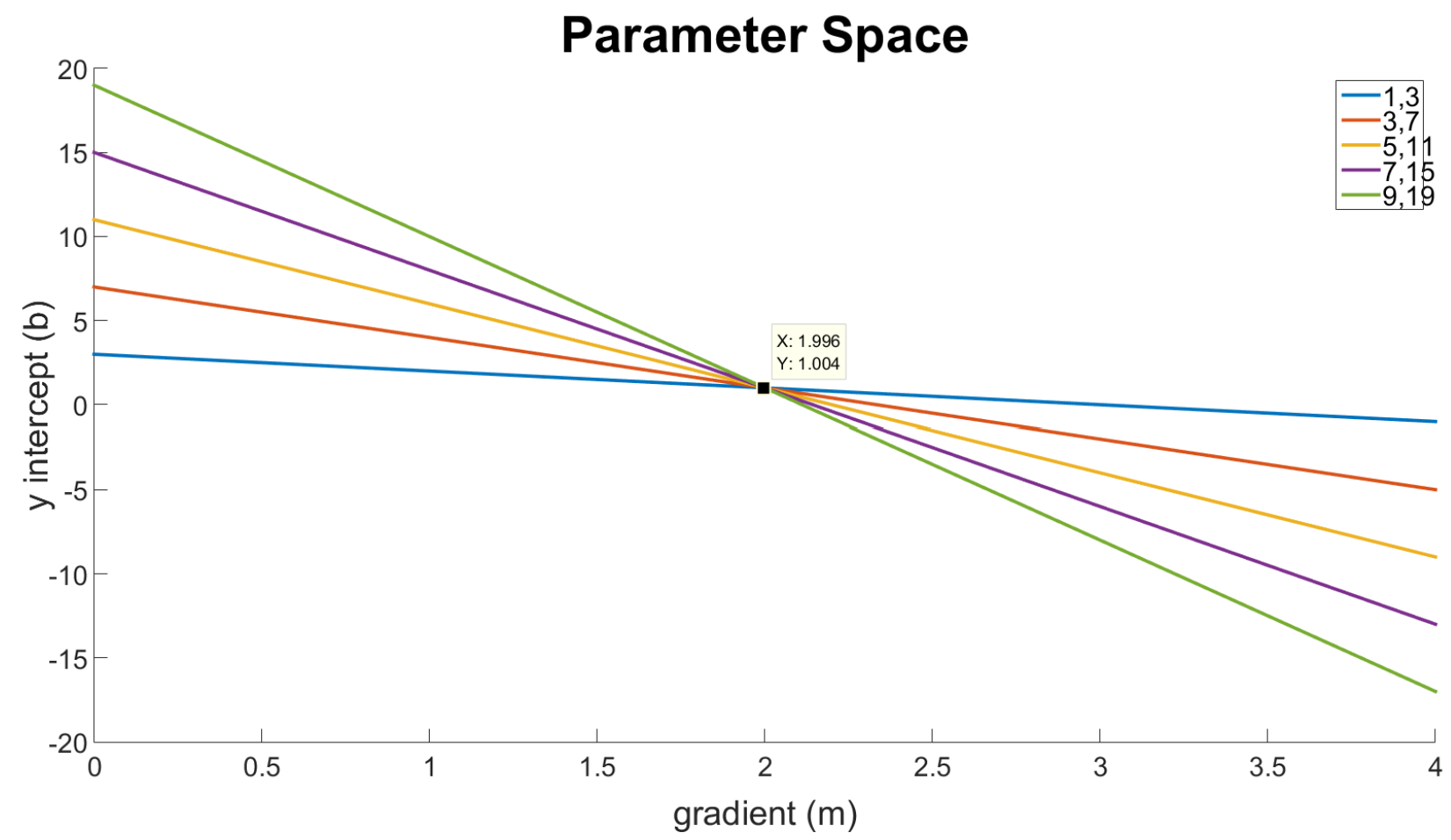

Figure 3-2: Parameter Space $(m=1.99, b=1.00)$ formed by 5 points taken from Feature Space 
However, a problem can be quickly noted with this approach. If there is a vertical line with an infinite gradient $\mathrm{m}$, it is hence unbounded. Therefore, the normal form for line extraction can be done using the polar coordinate using equation 3.1.

$r=0.5(x \cos \emptyset+y \sin \emptyset)$

Where $r$ is the distance from the origin to a given point $(\mathrm{x}, \mathrm{y})$ from the F.S. and $\varnothing$ or theta is the angle between the $\mathrm{x}$ axis and the line connecting from the origin to the point of interest $(\mathrm{x}, \mathrm{y})$. The combination of $\mathrm{r}$ and $\emptyset$ space is also referred to as the P.S. or Hough Space. All possible $\mathrm{r}$ are computed for any given point $(\mathrm{x}, \mathrm{y})$ from the F.S. while sweeping through theta such that a perpendicular line to $r$ passing through the point of interest is generated. This in return forms a sinusoidal curve in the P.S. for each sample in the F.S. as can be seen in Figure 3-3. 


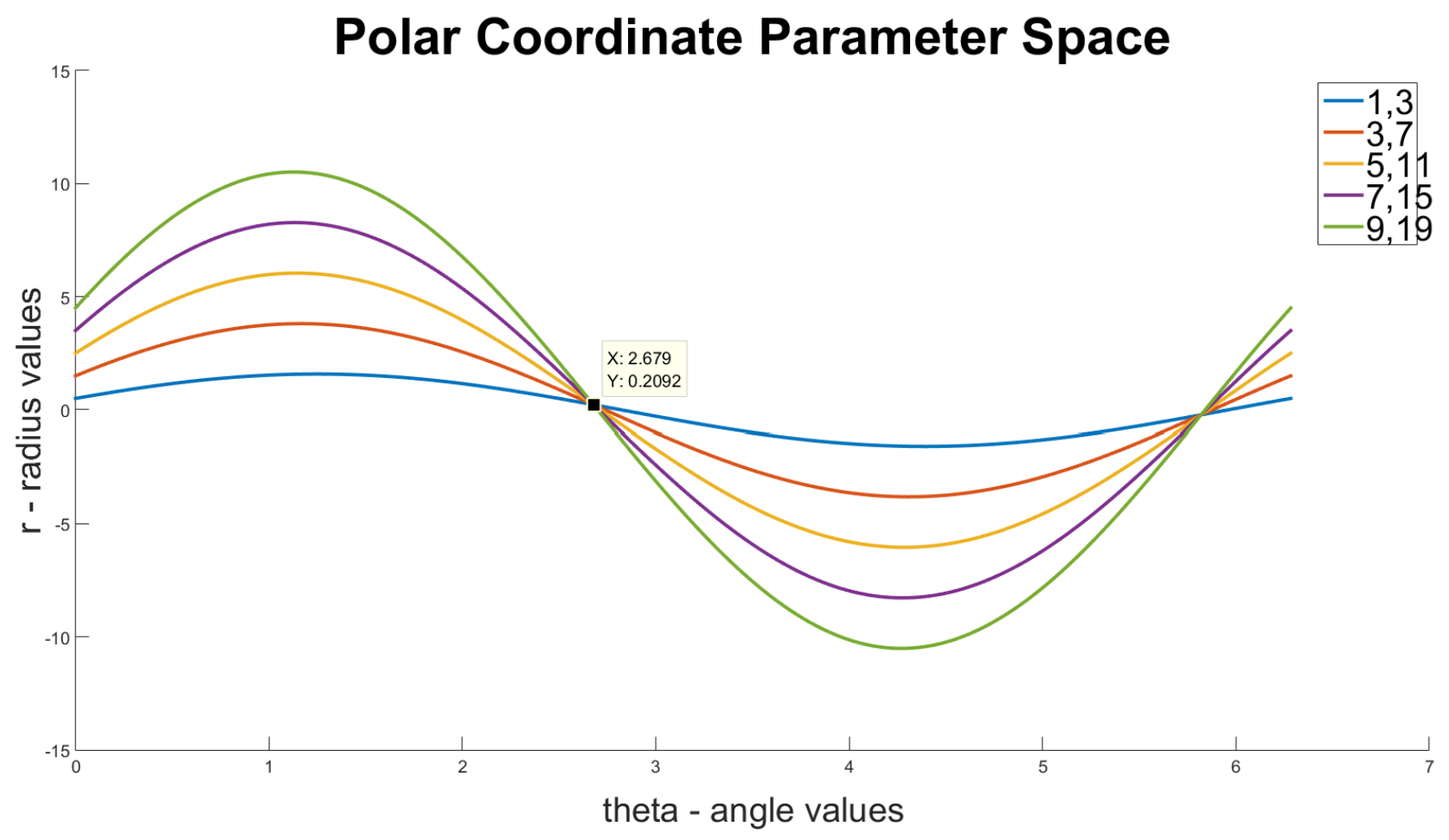

Figure 3-3: Parameter Space in polar coordinates $(\varnothing=2.68, r=0.21)$ represented by 5 samples

The intersection in the P.S. generates a peak which corresponds to a specific $\emptyset$ and $r$ hence the feature or line can be defined by following equation 3.2.

$y=-\frac{x}{\tan \varnothing}+\frac{2 * r}{\sin \varnothing}$

Where $-1 / \tan \emptyset$ corresponds to the gradient $m$ and $2 r / \sin \emptyset$ corresponds to the y intercept. Thus, using the P.S. value from Figure 3-3 and Equation 3.2, we get gradient 2.01 and yintercept 0.94 . A voting algorithm in terms of $\varnothing$ and $\mathrm{r}$ that is $\mathrm{A}(\varnothing, \mathrm{r})$ is able to determine the point at which the sinusoidal curves intercept by generating a peak due to votes accumulated during scan processes for each point in the F.S.. The voting algorithm can 
be represented by equation 3.3 given a multilateration measurement, for each $\mathrm{x}$ and $\mathrm{y}$ coordinate if a solution exists, we record a value of zero or very close to zero.

$A(\varnothing, \mathrm{r})=\sum_{\varnothing=-90}^{89} r(x, y \mid \varnothing)$

The accumulator array turns the line detection problem to a summit searching problem on the Hough Space or P.S. defined by $\emptyset$ and r. [18] Figure 3-3 demonstrates the concept of the accumulator array where a peak occurs at the intersection of the sinusoids.

The Generalized Hough Transform uses the same principles but adds new parameters to the Hough Space as per object shape. The Randomized Hough Transform ignores checking values of $\emptyset$ that are not common between points in the FS and hence reduces additional computation resulting in shorter time to resolve features within an image. I will be using a modified version of the Hough Transform for geolocation.

\subsection{Modified Hough Transform}

In order to use the Modified Hough Transform for geolocation, we will have to go through and map our problem to fit the Hough Transform properties. We will take the TDOA and FDOA examples where for a particular target location a time and frequency difference is produced. Because the target is moving, we will have one measurement of TDOA and one measurement of FDOA per target location which constitutes the F.S.. The P.S. in $\mathrm{x}$ and $\mathrm{y}$ can be defined so that accumulation of the voting array due to two or more measurements resulting in a peak in the accumulator array describes the location of the target that best fits the hyperbolic and elliptical lines of position due to measurements 
from the TDOA and the FDOA taken from the F.S. respectively. The measurements from the F.S. can refer to two or more different TDOA measurements due to different receiver pairs. The voting array is a table of normalized conditional probability centered on an event, in this case the multilateration measurements such as TDOA and FDOA. The accumulator array is a summation of one or more voting arrays such that a peak formed in the P.S. or H.T. space coincides with the likelihood of the emitter position.

A voting array is represented in equation 3.4 where for a given TDOA or FDOA measurement with error, we sweep through the P.S. $x$ and y potential position values of the target.

$A_{\text {voting }}(\mathrm{x}, \mathrm{y})=\sum_{x=\text { xlow }}^{x h i g h} \sum_{y=y \text { low }}^{\text {yhigh }} \mathrm{e}^{-f(\mathrm{x}, \mathrm{y} \mid \mathrm{T} / \mathrm{FDOAmeasurement})}$

where $f=\operatorname{DifferenceOf\operatorname {Arrival}(x,y)-T/FDOAmeasurement}+$ Merror

The voting array finds all possible $\mathrm{x}$ positions and y positions for a particular TDOA and FDOA measurement. Figure 3-4 and Figure 3-5 are an example of a voting array for TDOA and FDOA measurements respectively. Take note of the row of peaks in each figure; this represents all possible $\mathrm{x}$ and $\mathrm{y}$ values in the P.S. for the given measurement point in the F.S., namely the TDOA or/and FDOA measurements. The target is located at $(200,1000)$ and only 2 receivers were used to collect the multilateration data. 


\section{TDOA SURFACE PLOT of SCAN (Normalized)}

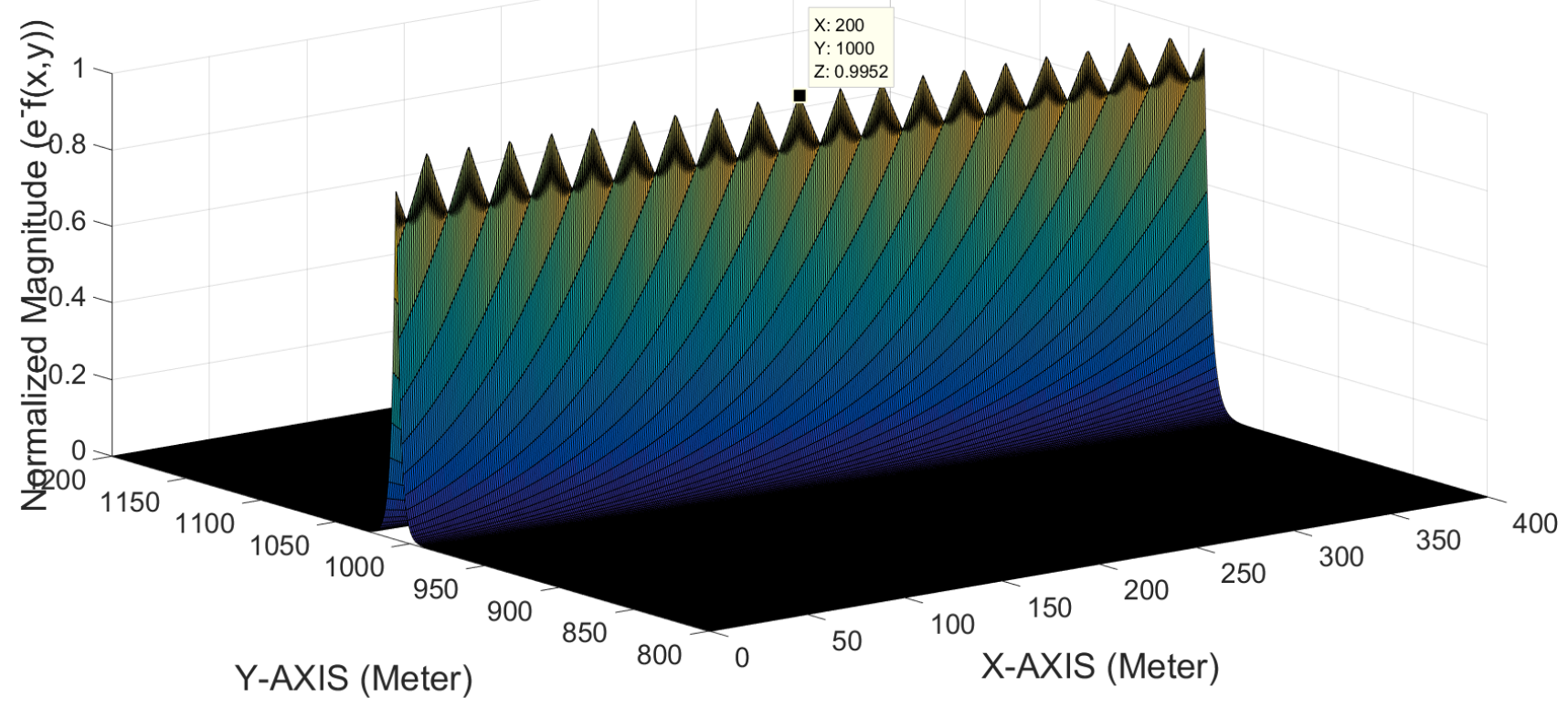

Figure 3-4 : TDOA plot of voting array in $x$ and $y$

\section{FDOA SURFACE PLOT of SCAN (Normalized)}

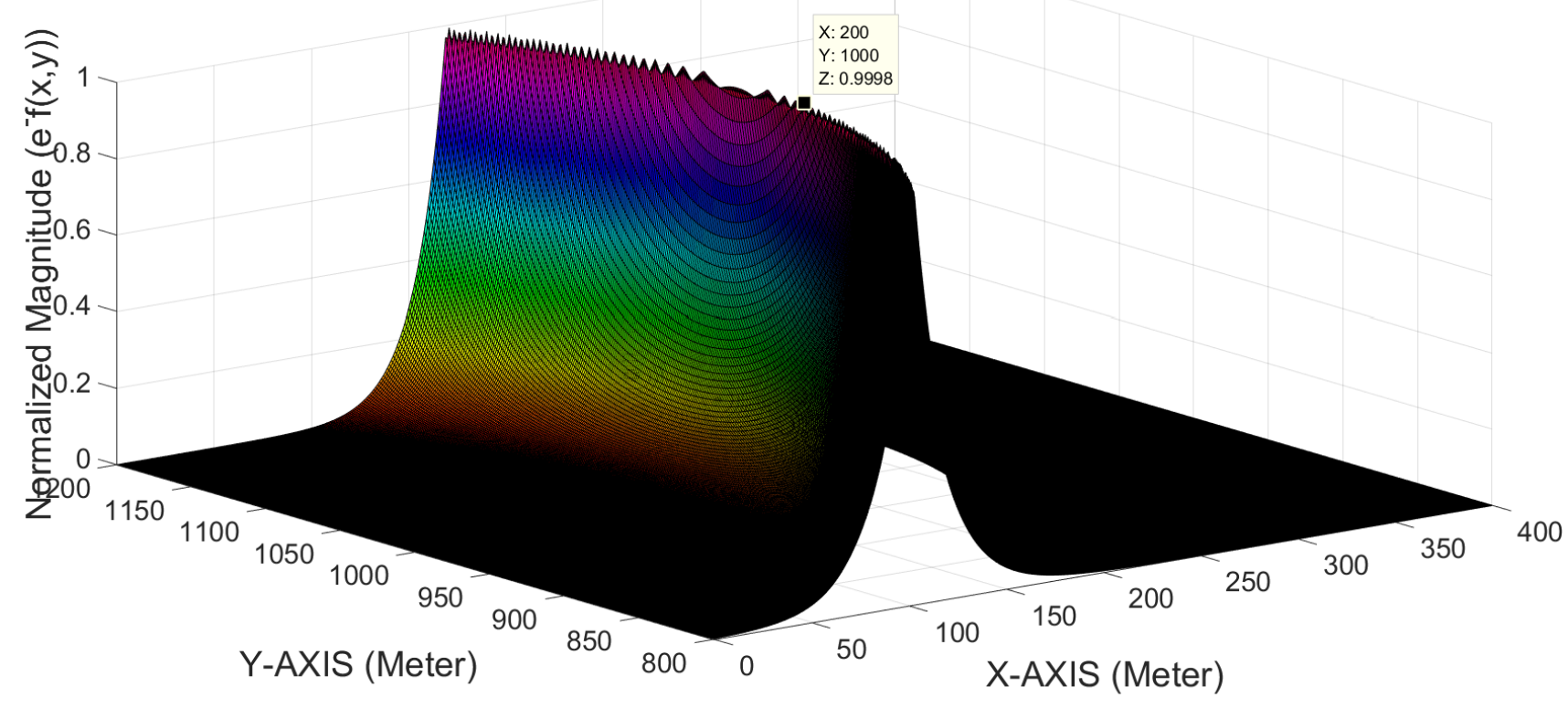

Figure 3-5 : FDOA plot of voting array in $x$ and $y$ 


\subsection{Data Fusion with Modified Hough Transform}

The nature of our experiment setup does not allow for fusion of a multiple voting array to further enhance the accuracy of our result. Will be using a filtering technique in chapter 4 to enhance results. What we do have in our experiment, however, is fusion of two measurements taken simultaneously, namely the TDOA and FDOA. The fusion of both these voting arrays is possible because the parametrized space is unified, irrespective of the measurement thus forming a strong peak that can be seen in Figure 3-6.

The accumulator array in Figure 3-6 gives the peak point $(200,1000)$ which satisfies both measurement points from the F.S.. This should be noted: the error measurement for FDOA will be orthogonal to the TDOA measurement which is evident when observing Figure 3-4 and Figure 3-5. 


\section{TDOA+FDOA SURFACE PLOT of SCAN (Normalized)}

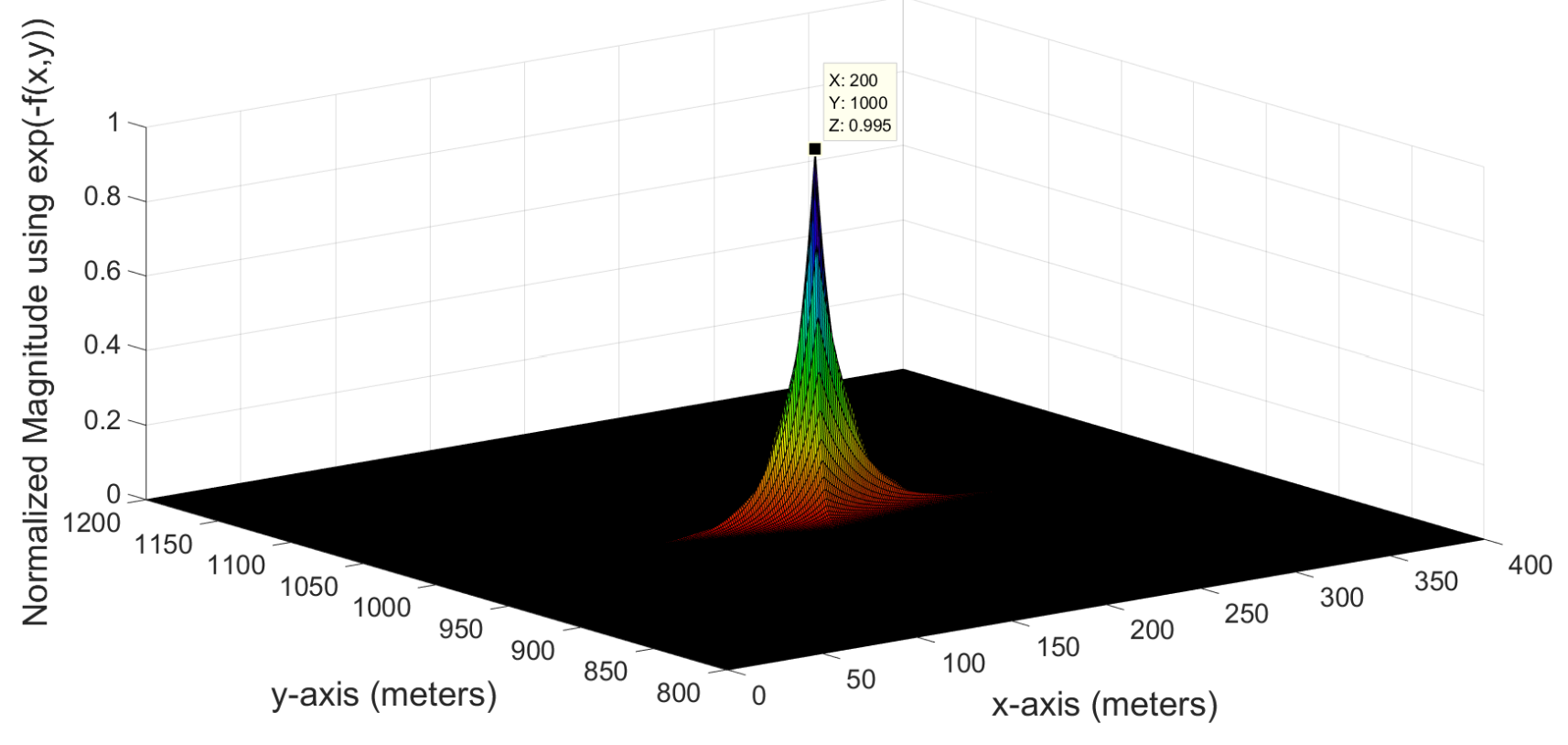

Figure 3-6 : TDOA and FDOA data fusion to form Accumulator Array

The accumulator array equation can be represented by Equation 3.5.

$A_{\text {accumulator }}(\mathrm{x}, \mathrm{y})=\sum_{i=1}^{N} A_{\text {voting }_{i}}(x, y), \mathrm{i}=1,2,3 \ldots \mathrm{N}$

The accumulator array consists of summation of at least 2 or more voting arrays that can come from multiple multilateration measurements. In our example above, we have used one TDOA measurement and one FDOA measurement. We will be making use of a third voting array using a TDOA measurement from a third receiver pair only to determine the velocity of target that will be used in the FDOA measurements.

\subsection{Geometric Dilution of Precision (GDOP)}

The Geometric Dilution of Precision (GDOP) is known as a ratio position error to ranging measurement error. "It represents the factor by which the fundamental ranging 
sensor error is magnified by the geometrical relationships between the emitter and each of the sensor platforms [12]." The GDOP, formed due to the target and each receiver pair will be unique. If there are multiple receiver pairs used to compute GDOP for the same target using sensor/receiver pair level fusion, the overall combined GDOP due to each receiver pair will be effected.

In its simpliset form, the dilution of precision can be derived by taking the trace of the covariance matrix $\mathrm{C}$, as shown in equation 3.6, using matrix A composed of unit vectors from the target to each receiver.

$C=\left(A^{T} A\right)^{-1}=\left(\begin{array}{cc}\sigma_{x}^{2} & \sigma_{x y} \\ \sigma_{x y} & \sigma_{y}^{2}\end{array}\right)$

where $A=\left(\begin{array}{cc}\frac{\left(x_{1}-x_{t}\right)}{\left\|r_{1}\right\|} & \frac{\left(y_{1}-y\right)}{\left\|r_{1}\right\|} \\ \frac{\left(x_{2}-x_{t}\right)}{\left\|r_{2}\right\|} & \frac{\left(y_{1}-y\right)}{\left\|r_{2}\right\|}\end{array}\right)$

Therefore, by taking the trace of matrix C, we result in the Position Dilution of Precision (PDOP). By adding the Time Dilution of Precision (TDOP), we are able to get the GDOP with the assumption that time measurement errors are identical, the zero mean and correlation error are zero between receivers. Equation 3.7 demonstrates the GDOP.

$G D O P=\sqrt{\left(\sigma_{x}^{2}+\sigma_{y}^{2}\right)+T D O P^{2}}$

where $T D O P=\sqrt{\sigma_{t}^{2}}$ 
TDOP can be extracted from equation 3.6 by using a $3^{\text {rd }}$ receiver and placing a negative one to a $3^{\text {rd }}$ column in matrix $\mathrm{A}$ such that matrix $\mathrm{C}$ will have a $3^{\text {rd }}$ row and column which will be $\mathrm{TDOP}^{2}$.

Another method of getting a quick estimate of GDOP is using downrange and crossrange GDOP measurements as a function of half the bistatic angle. To do this, we will be using Figure 3-7 to illustrate a multistatic radar using only two receivers: $V_{1}$ and $V_{2}$ with half the bistatic angle $\alpha$.

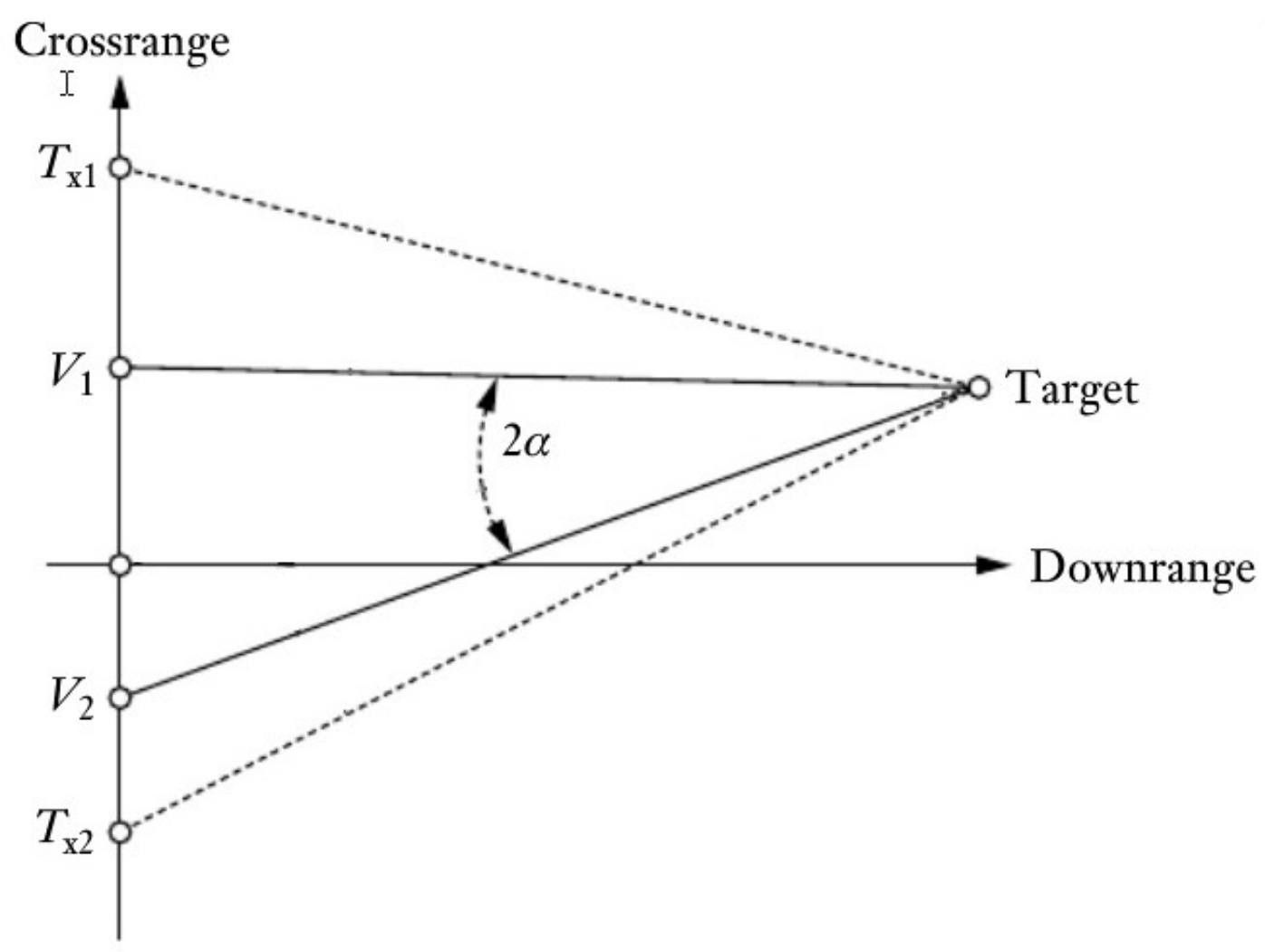

Figure 3-7: Demonstration of crossrange and downrange with 2 receivers [2] 
The downrange error $\sigma_{\mathrm{dr}}$ with the range error $\sigma_{\mathrm{r} 1}$ is due to receiver $\mathrm{V}_{1}$ and crossrange error $\sigma_{\mathrm{cr}}$ with range error $\sigma_{\mathrm{r} 2}$ is due to receiver $\mathrm{V}_{2}$, where both receivers are modeled as virtual monostatic radars as seen in Figure 3-8.

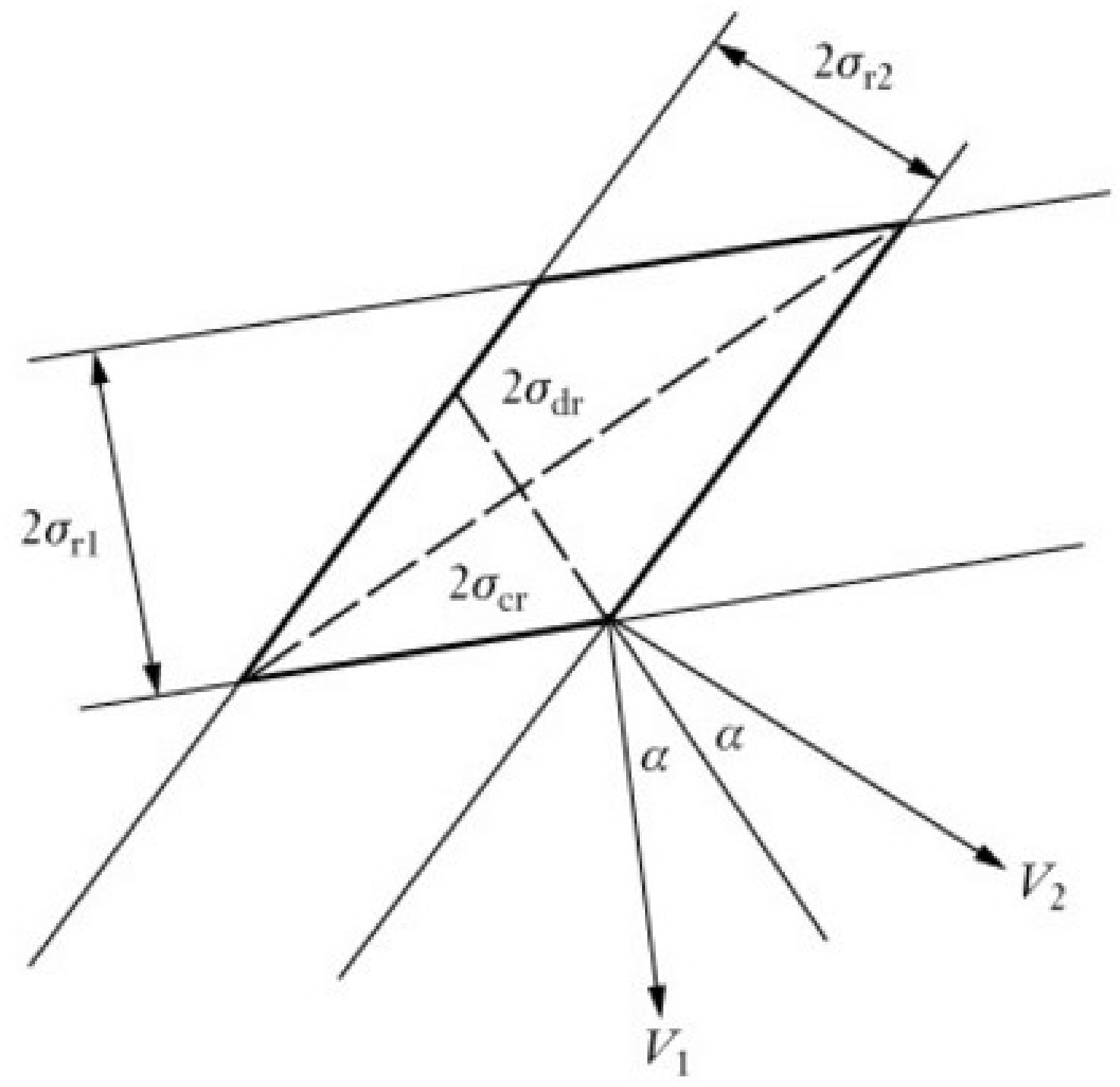

Figure 3-8: Multistatic error eclipse approximated as parallelogram for two virtual monostatic radar [2]

The bistatic GDOP factors can be represented by the fraction of the downrange error over the monostatic range error representing GDOP for FDOA measurements and the fraction 
of crossrange error over the monostatic range error representing GDOP for TDOA

measurements given the same setup with 2 receivers only. Thus, we have equation 3.8 representing the GDOP for a bistatic setup.

$G D O P_{d r}=\frac{\sigma_{d r}}{\sigma_{r 1}}=\frac{1}{\sqrt{2} \cos \alpha}$

and $G D O P_{c r}=\frac{\sigma_{c r}}{\sigma_{r 1}}=\frac{1}{\sqrt{2} \sin \alpha}$

with $\sigma_{r 1}=\frac{1}{B_{R M S} \sqrt{2 S N R}}$

The virtual monostatic range errors are taken to be equivalent and are dependent on SNR and signal band width. Equation 3.8 will be used in chapter 5 when representing simulation results to correlate measurements with GDOP values.

\subsubsection{GDOP Examples}

Table 3-1 demonstrates GDOP values using equation 3.6 for various multistatic radar setups demonstrating that the lower the GDOP value, the better the geometry.

Table 3-1 : GDOP measurements for different multistatic radar setup

\begin{tabular}{|l|l|l|l|l|}
\hline Target $(\mathrm{x}, \mathrm{y})$ & $\mathrm{Rx} 1$ & $\mathrm{Rx} 2$ & $\mathrm{Rx}$ (if any) & GDOP \\
\hline 0,0 & 20,20 & 30,0 & $-10,-20$ & 1.46 \\
\hline 0,10 & $-10,0$ & 10,5 & $\mathrm{~N} / \mathrm{A}$ & 3.87 \\
\hline 100,100 & 20,20 & 30,0 & $-10,-20$ & 7.80 \\
\hline 100,100 & $-10,0$ & 10,5 & N/A & 18.97 \\
\hline
\end{tabular}

A setup including 4 plots that have bad/good GDOP, noisy/clean TDOA and FDOA measurements is presented. The target is located at (100,500). From Figure 3-9 and 3- 
10, we can see that having a good GDOP setup relative to Figure 3-11 and 3-12 with bad GDOP is important. This is because a bad GDOP setup is much more sensitive to noise.

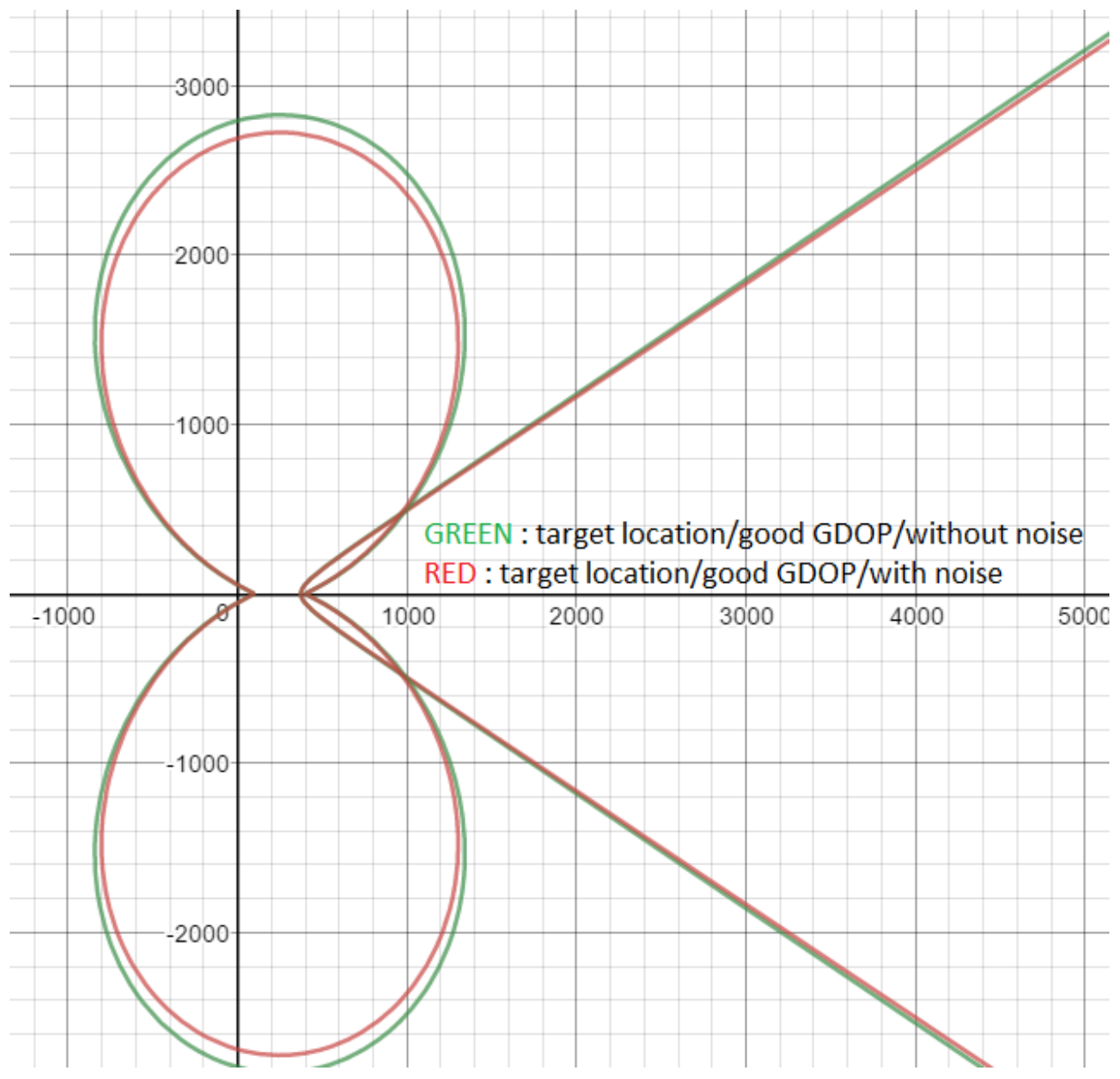

Figure 3-9: Good GDOP FDOA and TDOA with and without noise 


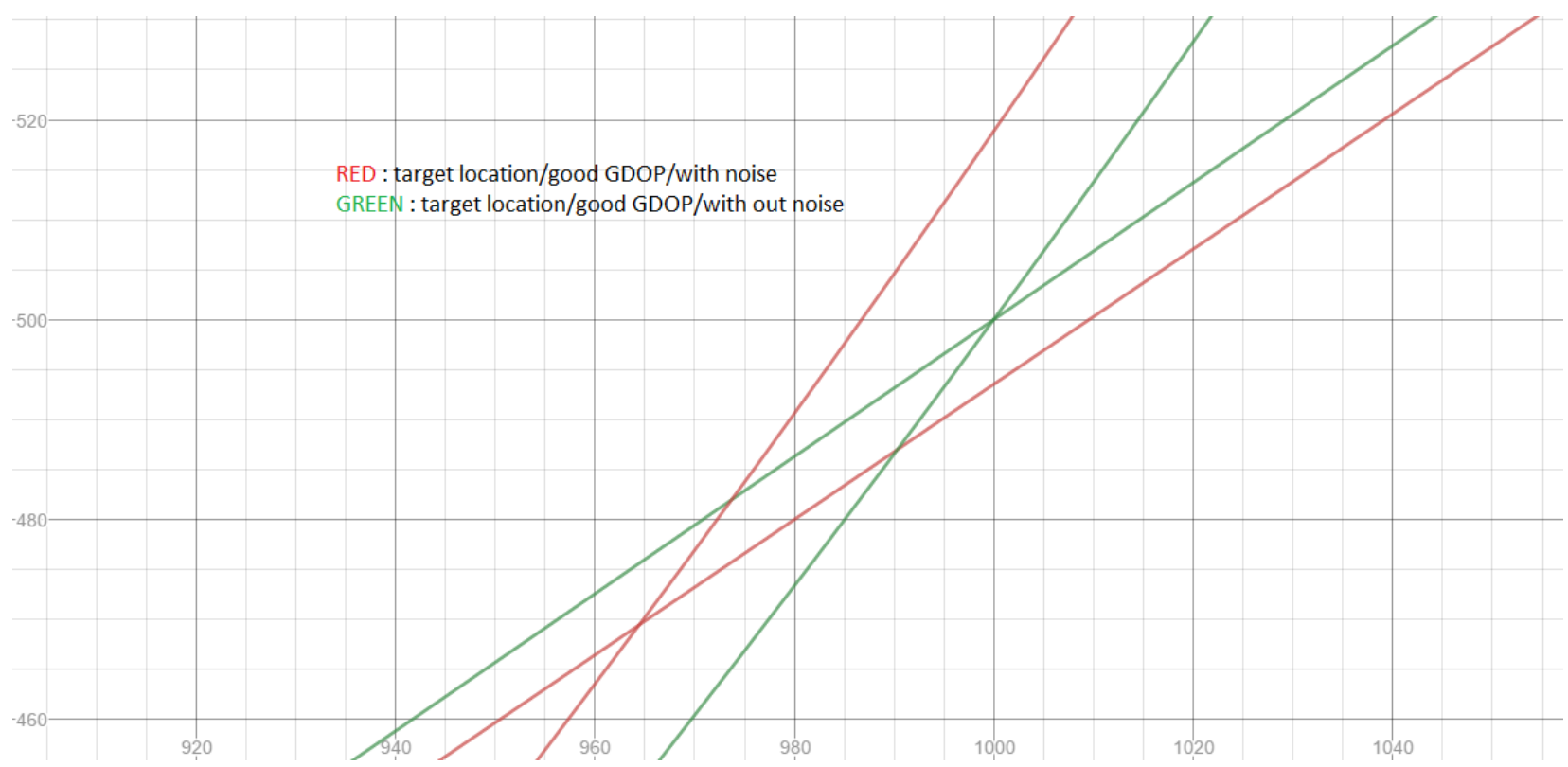

Figure 3-10 : Target located $(1000,500)$ without noise and $(964,468)$ with noise

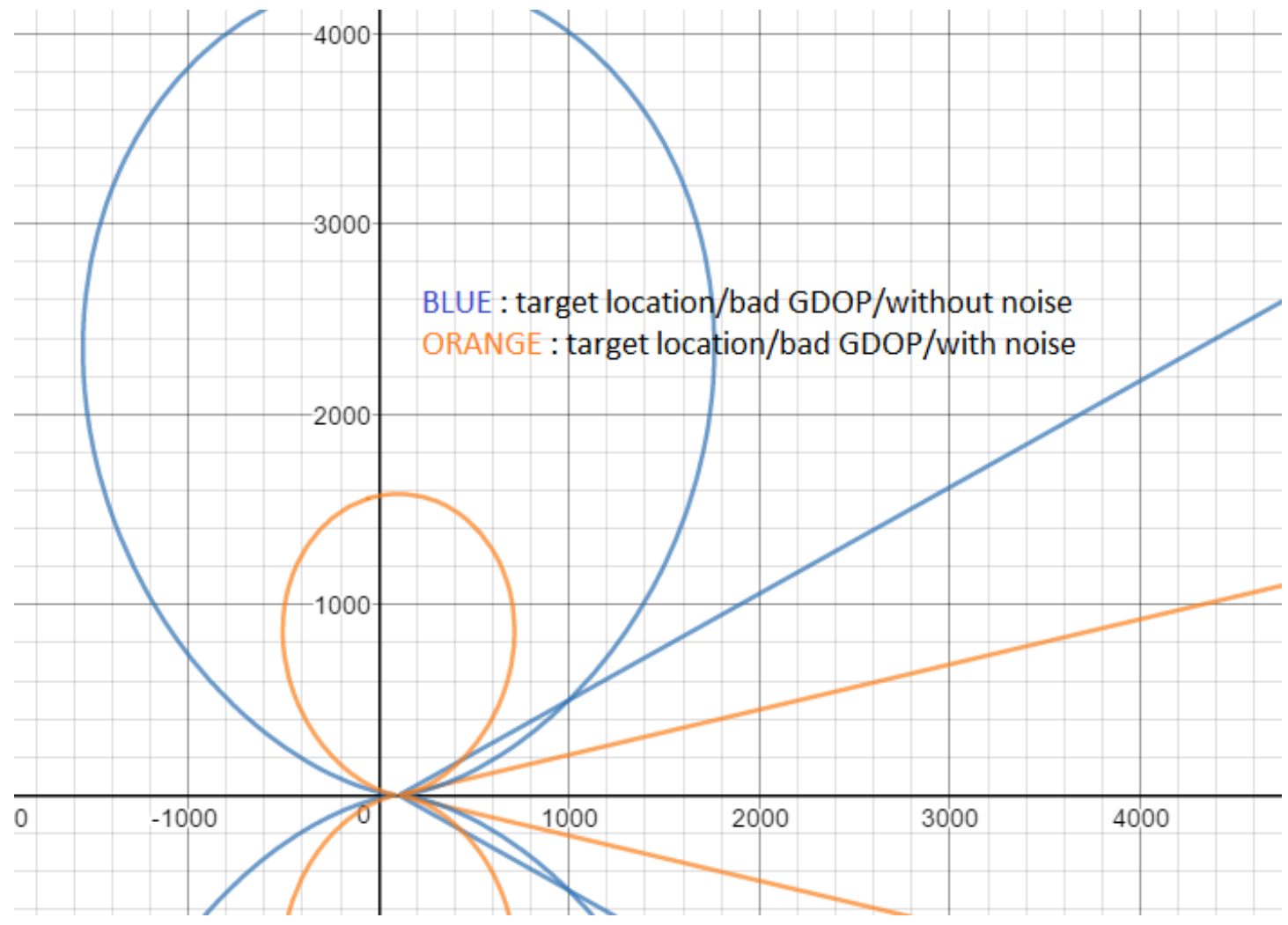

Figure 3-11 : Bad GDOP FDOA and TDOA with and without noise 


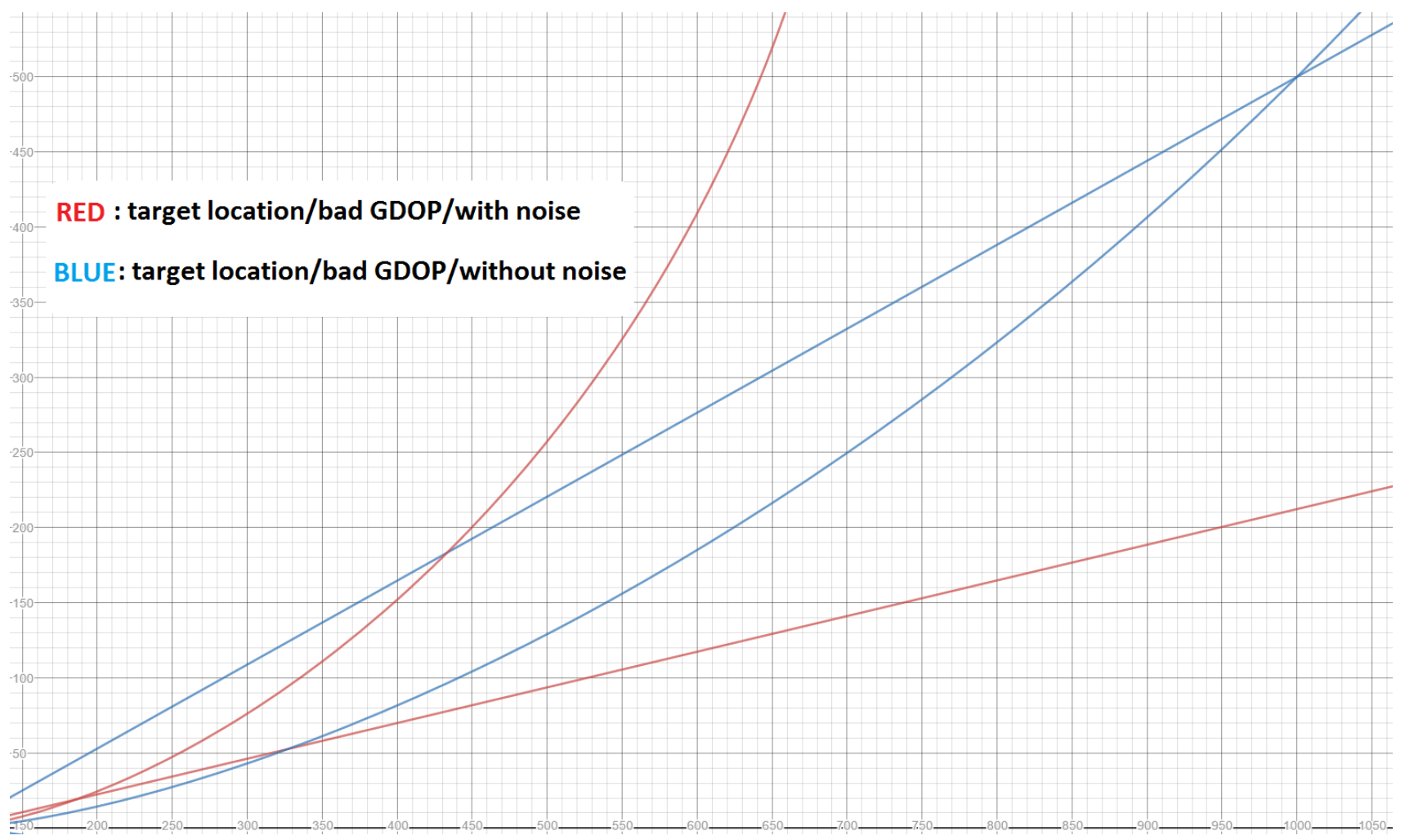

Figure 3-12: Target located $(1000,500)$ without noise and $(185,20)$ with noise both with bad GDOP

Observing from Figure 3-10 and Figure 2-12 that are close up views of Figure 3-9 and Figure 3-11 respectively. The noisy measurement due to a good $(964,468)$ and bad $(185,20)$ GDOP setup, we can see a huge difference between them with the bad GDOP measurement being very far off the true target location $(1000,500)$. 


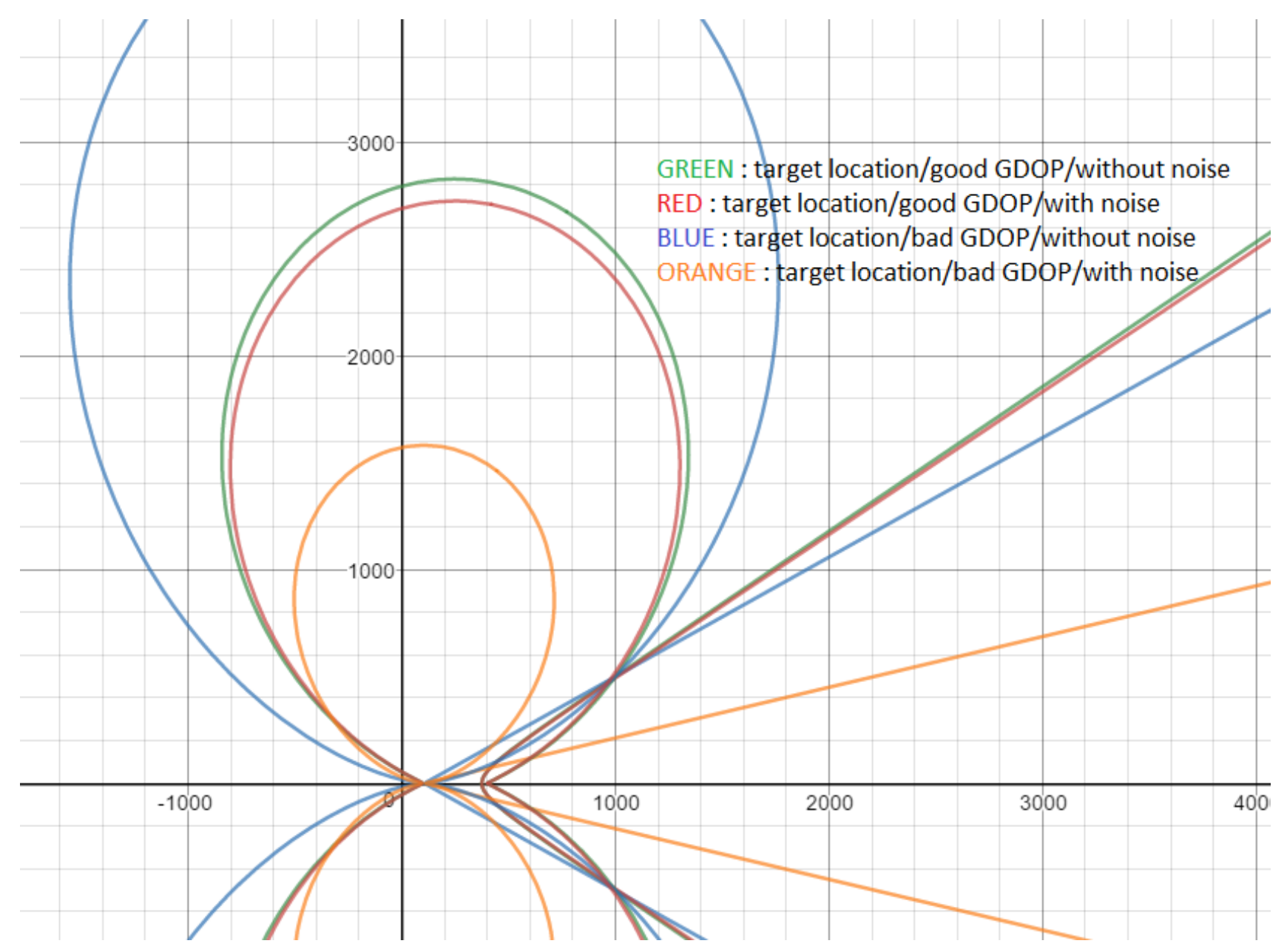

Figure 3-13 : Bad GDOP is more sensitive to noise than Good GDOP setup

Figure 3-13 overlays graphs 3-9 and 3-11 showing the sensitivity to the same level of TDOA and FDOA measurement noise of the bad GDOP setup. Therefore ensuring good GDOP is important while using the multilateration technique.

\subsection{Chapter Summary}

In this chapter, an explanation of the Hough Transform is covered then modified it to fit the geolocation needs using the multilateration technique. We then followed through with data fusion of different multilateration technique measurements such as TDOA and 
FDOA. The importance of GDOP with examples concluded the chapter indicating poor GDOP is more sensitive to noise variations. In the following chapter, a filtering technique will help mitigate noisy measurements from the modified Hough Transform. 


\section{Chapter 4 Filtering Techniques}

The Modified Hough Transform provides an initial guess for target location using only TDOA measurements with 3 receivers that are refined by Kalman Filtering (KF) over multiple samples to get a good estimate of target velocity. Assuming the target velocity remains unchanged, we can now compute the target location using 2 receivers with multilareation techniques that include TDOA and FDOA where the error hyperbola and ellipse are perpendicular thus minimizing sensitivity when GDOP is good. The target location measurements will be noisy, so filtering the noise by using the Kalman Filter assuming linearity over a short sample size will reduce the RMSE. This Chapter will cover why Kalman Filter is needed and how to use it for the purpose of this thesis.

\subsection{Sources of Noise}

Sources of noise can be inherent in the equipment used or due to errors in measurements taken. The sources which can constitute noise in the overall system are the measurement noise, multipath noise, GPS synchronization noise, receiver location noise, modelling noise, target velocity, noise due to rain and thunderstorms [13]. In our application, knowing which sources of noise effect the multilateration equations such as the TDOA will help in making assumptions and noise modeling. The main sources for the purpose

of this thesis are the TDOA measurement noise and FDOA measurement noise, velocity of the target used in the FDOA multilateration equation and the location of the receivers. 
In this chapter, we will be focusing on measuring the noise of TDOA and FDOA as the major source of noise using the Cramer-Rao Lower Bounds (CRLB). We will assume the standard deviation noise are zero-mean Guassian. As for multipath noise, we can mitigate this with the assumption that the experiment is performed on a flat plane. Synchronization noise affects the TDOA measurement mainly, this will be modeled using CRLB in the next section. The noise due to receiver location will affect both the FDOA and TDOA measurements, however because the receivers are stationary, an assumption will be made that the source of noise due to receiver location is insignificant.

Noise due to clutter such as weather conditions or chaff will be assumed to be insignificant. Assumption that no multi-target tracking will take place that can add complexity if not taken into account [11]. The target velocity noise will affect the FDOA measurements only. However, we will demonstrate that, using a dual stage method with TDOA only measurements and the Kalman Filter to refine the value of the target velocity, (which will then be used in the FDOA measurement along with TDOA), the target can be located using only two receivers.

\subsubsection{Measurement Noise}

The Fisher information matrix (FIM) provides a fundamental estimation limit for unbiased estimators which is referred to as the Cramer-Rao Lower Bound (CRLB). The FIM $J(k)$ is defined by Equation 4.1 and 4.2.

$$
\begin{aligned}
& J(k)=E\left(\nabla_{k}^{T} \ln p_{E}(T F D O A-f(k)) \nabla_{k}\right) \ln p_{E}(T F D O A-f(k)) \\
& \nabla_{k} \ln p_{E}(T F D O A-f(k))=\frac{d \ln p_{E}(T F D O A-f(k))}{d x} \frac{d \ln p_{E}(T F D O A-f(k))}{d y}
\end{aligned}
$$


Where $\mathrm{k}=(\mathrm{x}, \mathrm{y})$ the position of the target location, and TFDOA is the measure of TDOA or FDOA based on $\mathrm{f}(\mathrm{k})$ measurement. The likelihood given error distribution is represented by $\mathrm{p}_{\mathrm{E}}(\mathrm{TFDOA}-\mathrm{f}(\mathrm{k}))$. With Gaussian measurement error $\mathrm{p}_{\mathrm{E}}(\mathrm{error})=$ $\mathrm{N}(0, \mathrm{~N}(\mathrm{k}))$ where $\mathrm{N}(\mathrm{k})$ is the measurement error covariance matrix, the FIM can now be represented by Equation 4.3 and 4.4.

$J(k)=Q^{T}(k) N(k)^{-1} Q(k)$

$Q(k)=\nabla_{k}(f(k))$

In general, we need numerical methods to evaluate CRLB. "The larger the gradient $\mathrm{Q}(\mathrm{k})$, or the smaller the measurement error, the more information is provided from the measurement and the smaller the potential estimation error." [12] In the Gaussian case with a diagonal measurement error covariance matrix, it is important to note that the trace of the FIM is the squared gradient magnitude. The CRLB is given by Equation 4.5.

$$
\operatorname{Cov}(\hat{k})=E(k-\hat{k})(k-\hat{k})^{T} \geq J^{-1}(k)
$$

where $\mathrm{k}$ denotes the true position and the $\hat{k}$, the estimated position. The CRLB holds for any unbiased estimate of $\hat{k}$. By using CRLB for active sensors, we get the following lower bound limits that can be used to estimate a measurement noise for TDOA and FDOA measurements. For the TDOA, the CRLB expression is as follows in Equation 4.6. [11]

$\sigma_{T D O A} \geq \frac{1}{2 \Pi B_{R M S} \sqrt{B T S_{\text {eff }}}}$ 
For the FDOA, the CRLB expression is as follows in Equation 4.7. [11]

$\sigma_{F D O A} \geq \frac{1}{2 \Pi T_{R M S} \sqrt{B T S_{e f f}}}$

The noise bandwidth of the receiver is $\mathrm{B}$ and the collection time $\mathrm{T}$ thus results in the product BT which is the time bandwidth product that results from the discrete number of samples. The effective signal to noise ratio is given by Equation 4.8

$S_{e f f}=\frac{1}{\frac{1}{S N R_{1}}+\frac{1}{S N R_{2}}+\frac{1}{S N R_{1} S N R_{2}}} \approx \min \left\{S N R_{1}, S N R_{2}\right\}$

and the effective bandwidth $\mathrm{B}_{\mathrm{RMS}}$ is given by Equation 4.9. If the signal $\mathrm{S}(\mathrm{f})$ is a rectangle then the effective bandwidth is essentially the bandwidth of the rectangular signal itself.

$B_{R M S}^{2}=\frac{\int f^{2}|S(f)|^{2} d f}{\int|S(f)|^{2} d f} \quad$, where $\mathrm{S}(\mathrm{f})$ is the received signal

and the effective duration $\mathrm{T}_{\mathrm{RMS}}$ of the signal is computed using Equation 4.10. If the received signal has a constant envelope duration, then this in return is the effective duration.

$T_{R M S}^{2}=\frac{\int t^{2}|S(t)|^{2} d t}{\int|S(t)|^{2} d t} \quad$, where $\mathrm{S}(\mathrm{t})$ is the received signal

Perhaps the root mean square error (RMSE) is in effect of more importance. One way to interpret this is to view it as the achieved position error in meters. One implication of the CRLB is the following bound seen in Equation 4.11 [12] 
$R M S E=\sqrt{E\left[(k-\hat{k})^{2}\right]} \geq \sqrt{\operatorname{tr} \operatorname{Cov}(\hat{k})} \geq \sqrt{\operatorname{tr} J^{-1}(k)}$

The CRLB is related to the GDOP through the RMSE, that is, the RMSE normalized by the measurement accuracy. The RMSE will be used to determine enhancement in the location accuracy of the target and the CRLB will be used to get a lower bound to measurement error due to TDOA and FDOA so it can be used in a simulation model to inject noise.

\subsection{Kalman Filtering}

The Kalman filter, developed in 1960, has seen many applications in various fields such as aerospace, navigation, nuclear power plant instrumentation and many others. The Kalman filter, being a recursive one, minimizes the least-squares error. Target dynamics are directly used for the optimization of filter parameters. Kalman filter theory is made complex due to the complicated control theories. [12]

For simplicity, assume we have a one-dimensional problem. The target's velocity is constant. The target equations of motion can be seen in Equation 4.12.

$x_{n+1}=x_{n}+T \dot{x_{n}}$

with $\dot{x}_{n+1}=\dot{x}_{n}$

where at time $\mathrm{n}, \mathrm{x}_{\mathrm{n}}$ is the target location and $\dot{x}_{n}$ is the target velocity, and $\mathrm{T}$ is the update or sampling time. 
In the real world, the target will not have a constant velocity for all time. There is actually uncertainty in the target trajectory and target acceleration at any given time. The Kalman filter allows for this uncertainty in target motion by adding a random component to the target dynamics. The equations for the target dynamics are represented in Equation 4.13.

$x_{n+1}=x_{n}+T \dot{x_{n}}$

with $\dot{x}_{n+1}=\dot{x}_{n}+u_{n}$

where $u_{n}$ is a undermined or random change in velocity from time $n$ to time $n+1$. In matrix notation, the system dynamic equation is represented by Equation 4.13.

$X_{n+1}=A X_{n}+B U_{n}$

where $X_{n}=\left[\begin{array}{l}x_{n} \\ \dot{x}_{n}\end{array}\right]$ (state vector)

$$
\begin{aligned}
& U_{n}=\left[\begin{array}{l}
0 \\
u_{n}
\end{array}\right] \text { (control vector, dynamic model driving noise vector) } \\
& A=\left[\begin{array}{ll}
1 & T \\
0 & 1
\end{array}\right] \text { (state transition matrix) } \\
& B=\left[\begin{array}{ll}
0 & 0 \\
0 & 1
\end{array}\right] \text { (control matrix) }
\end{aligned}
$$

The measurement equation or the observation system equation is given by Equation 4.14.

$$
Y_{n}^{*}=Z_{n}-M X_{n, n-1}^{*}
$$

where $M=\left[\begin{array}{ll}1 & 0\end{array}\right]$ (observation or measurement matrix)

$$
X_{n, n-1}^{*}(\text { state prediction, from Equation 4.15) }
$$




$$
\begin{aligned}
& Z_{n} \text { (measurement matrix) } \\
& Y_{n}^{*} \text { (observation error) }
\end{aligned}
$$

The prediction equation is represented by Equation 4.15.

$$
X_{n, n-1}^{*}=A X_{n-1, n-1}^{*}+B U_{n}
$$

$$
\begin{gathered}
\text { where } X_{n-1, n-1}^{*}=\left[\begin{array}{l}
x_{n-1, n-1}^{*} \\
\dot{x}_{n-1, n-1}^{*}
\end{array}\right] \text { (state vector) } \\
X_{n, n-1}^{*}=\left[\begin{array}{l}
x_{n, n-1}^{*} \\
\dot{x}_{n, n-1}^{*}
\end{array}\right] \text { (state predicted vector) }
\end{gathered}
$$

The Kalman filtering equation is represented by Equation 4.16.

$$
X_{n, n}^{*}=X_{n, n-1}^{*}+K Y_{n}^{*}
$$

The matrix K, is known as the weight equation or Kalman Filter (K.F.) gain. It contains the filter weights for smoothing the measurement data and predicting the next position. This KF gain equation is represented by Equation 4.17.

$K_{n}=P_{n, n-1}^{*} M^{T}\left[M P_{n, n-1}^{*} M^{T}+R\right]^{-1}$

where $P_{n, n-1}^{*}=A P_{n-1, n-1}^{*} A^{T}+Q$ (covariance prediction)

$$
\begin{aligned}
& Q=\operatorname{COV}\left[U_{n}\right]=E\left[U_{n} U_{n}^{T}\right] \text { (dynamic model noise) } \\
& P_{n, n-1}^{*}=\operatorname{COV}\left[X_{n, n-1}^{*}\right]=E\left[X_{n, n-1}^{*} X_{n, n-1}^{* T}\right] \text { (initial covariance prediction) } \\
& R=\operatorname{COV}\left[N_{n}\right]=E\left[N_{n} N_{n}^{T}\right] \text { (observation noise covariance) } \\
& P_{n, n}^{*}=\operatorname{COV}\left[X_{n, n}^{*}\right]=(I-K M) P_{n, n-1}^{*} \text { (corrector equation) }
\end{aligned}
$$


The KF update can be broken into two major stages, the first being the time update or prediction. This is where we predict the state ahead along with its error covariance. The next stage is the measurement update or correction. This is where we compute the KF gain and update the state estimate and error covariance. This is illustrated in Figure 4.1with stages 1 and 2.

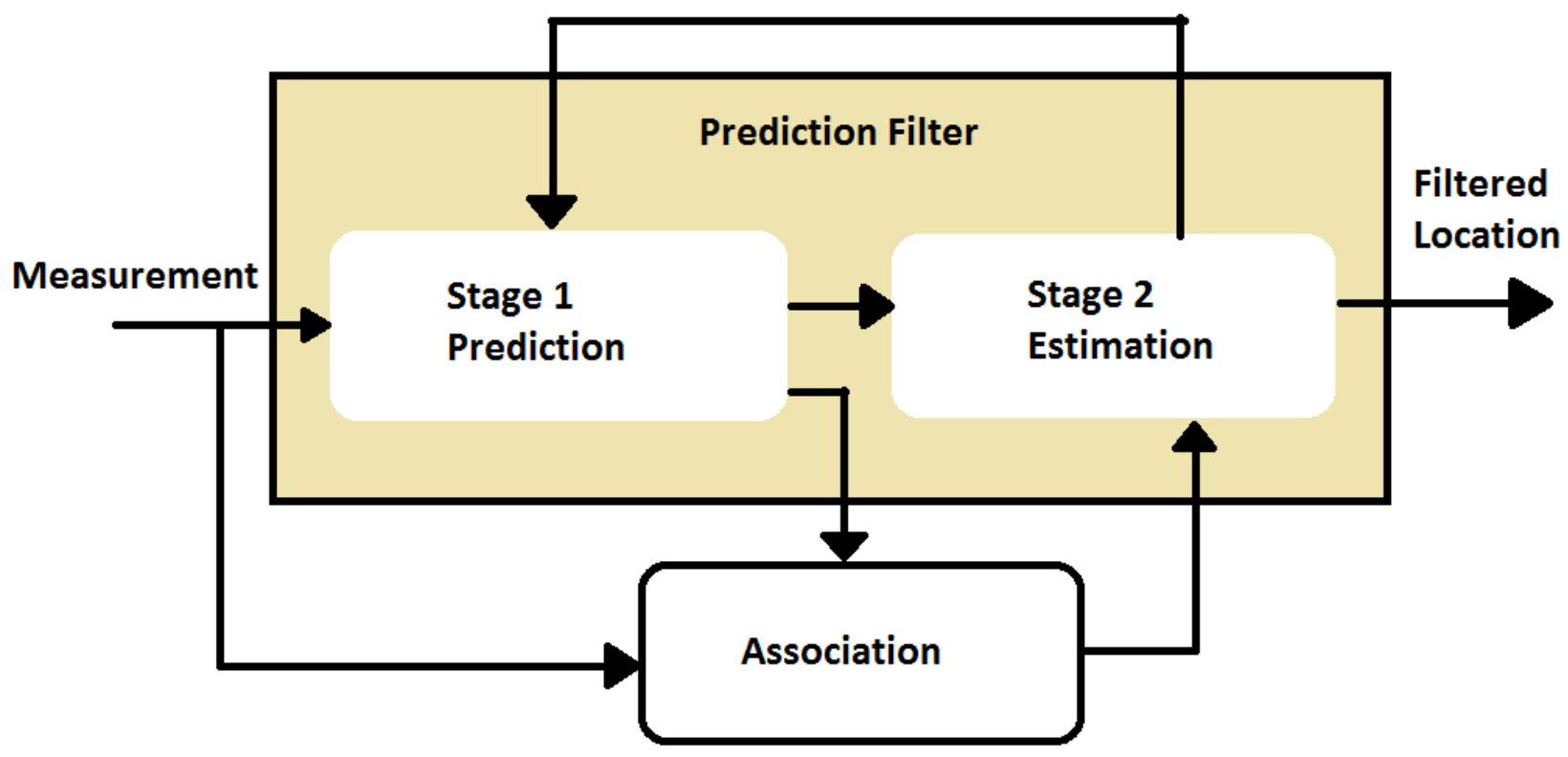

Figure 4-1 : Kalman Filter prediction flow diagram

Once the initialization of the covariance matrix is completed, the Kalman filter is implemented by the following five simple steps for each new measured position value.

Step 1. Apply the filtered position and velocity values to predict the next position and next velocity.

$X_{n, n-1}^{*}=A X_{n-1, n-1}^{*}$

Step 2. Project the covariance matrix. 


$$
P_{n, n-1}^{*}=A P_{n-1, n-1}^{*} A^{T}+Q
$$

Step 3. Compute the Kalman filter weighting equation to determine the filter weights.

$$
K_{n}=P_{n, n-1}^{*} M^{T}\left[M P_{n, n-1}^{*} M^{T}+R\right]^{-1}
$$

Step 4. Apply the KF gain to filter the position and velocity. Update estimate based on $Z_{n}$

$$
X_{n, n}^{*}=X_{n, n-1}^{*}+K\left(Z_{n}-M X_{n, n-1}^{*}\right)
$$

Step 5. Update the covariance matrix and time stamp $n=n+1$

$$
P_{n, n}^{*}=\operatorname{COV}\left[X_{n, n}^{*}\right]=\left(I-K_{n} M\right) P_{n, n-1}^{*}
$$

A demonstration of the Kalman Filter prediction filter can be observed using one dimension in Figure 4-2. We are assuming velocity remains constant between samples. 


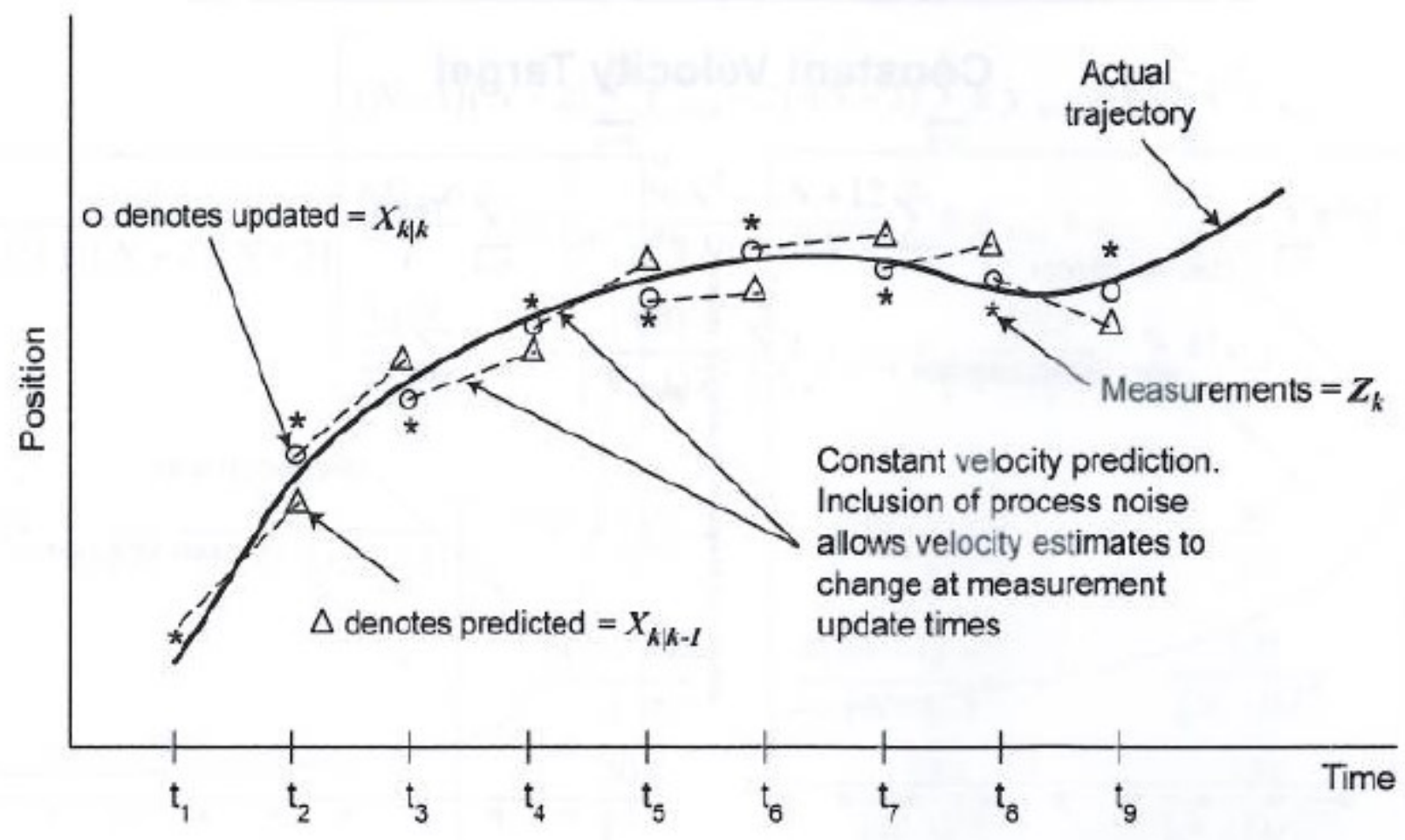

Figure 4-2 : Kalman Filter prediction in one dimension [21]

From Figure 4-2 we can see that a prediction was made at $t_{2}$ based on measured data at $t_{1}$.

The updated location at $\mathrm{t}_{2}$ is based on prediction and new measurement data at $\mathrm{t}_{2}$.

We then use our new best estimate at $t_{2}$ which is the updated location to form a prediction at $\mathrm{t}_{3}$. The new updated location at $\mathrm{t}_{3}$ is then based off of the prediction and new measurement data at $t_{3}$. The process repeats itself for latter samples.

\subsubsection{Velocity Prediction with Kalman Filtering}

Below in Figure 4-3 is an example of KF applied on a moving target in one dimension with Gaussian noise. It can be seen that the KF value approaches closer to the actual position as time progresses. 


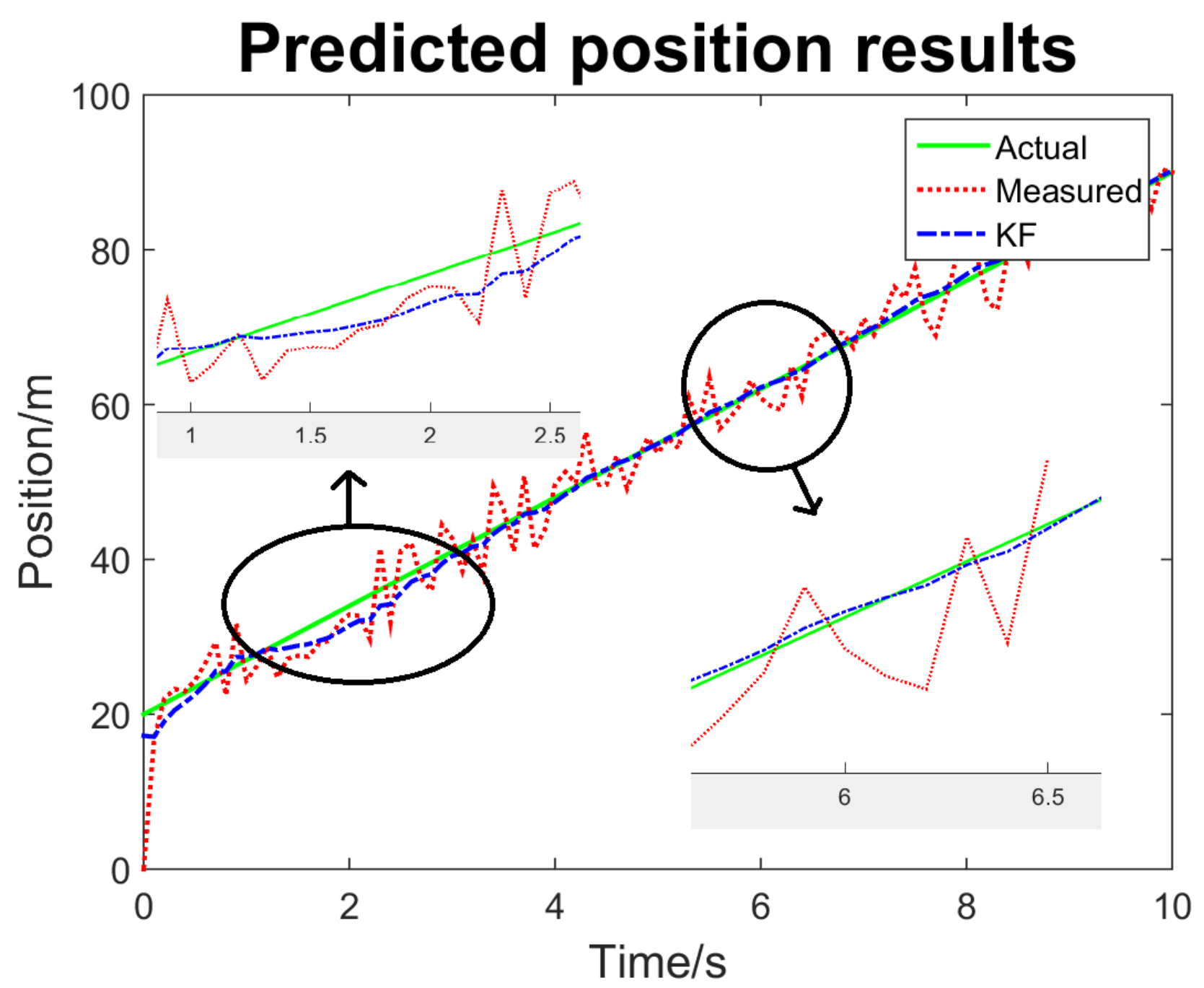

Figure 4-3: KF on target moving in $1 D$

Assuming the moving target has no acceleration and the velocity remains steady, meaning there is no drastic change in the direction of motion of the target. We are also going to make an initial guess on the velocity of the target based on several prior target location measurements. This filtering technique can be applied on position data gathered via multilateration technique provided there is no dependence on velocity data. This will be presented in the next chapter where dual stage method will be used to transition from a three receiver down to a two receiver mutistatic radar setup. 
The experiment setup has the following parameters is presented in table 4-1.

Table 4-1: Experiment setup parameter in 1D

\begin{tabular}{|c|c|}
\hline Initial Location & $20 \mathrm{~m}$ \\
\hline Sample Time & $0.1 \mathrm{~s}$ \\
\hline True Velocity & $7 \mathrm{~m} / \mathrm{s}$ \\
\hline
\end{tabular}

The RMSE of the measured data that has Gaussian noise relative to the actual location data can be seen in Figure 4-4.

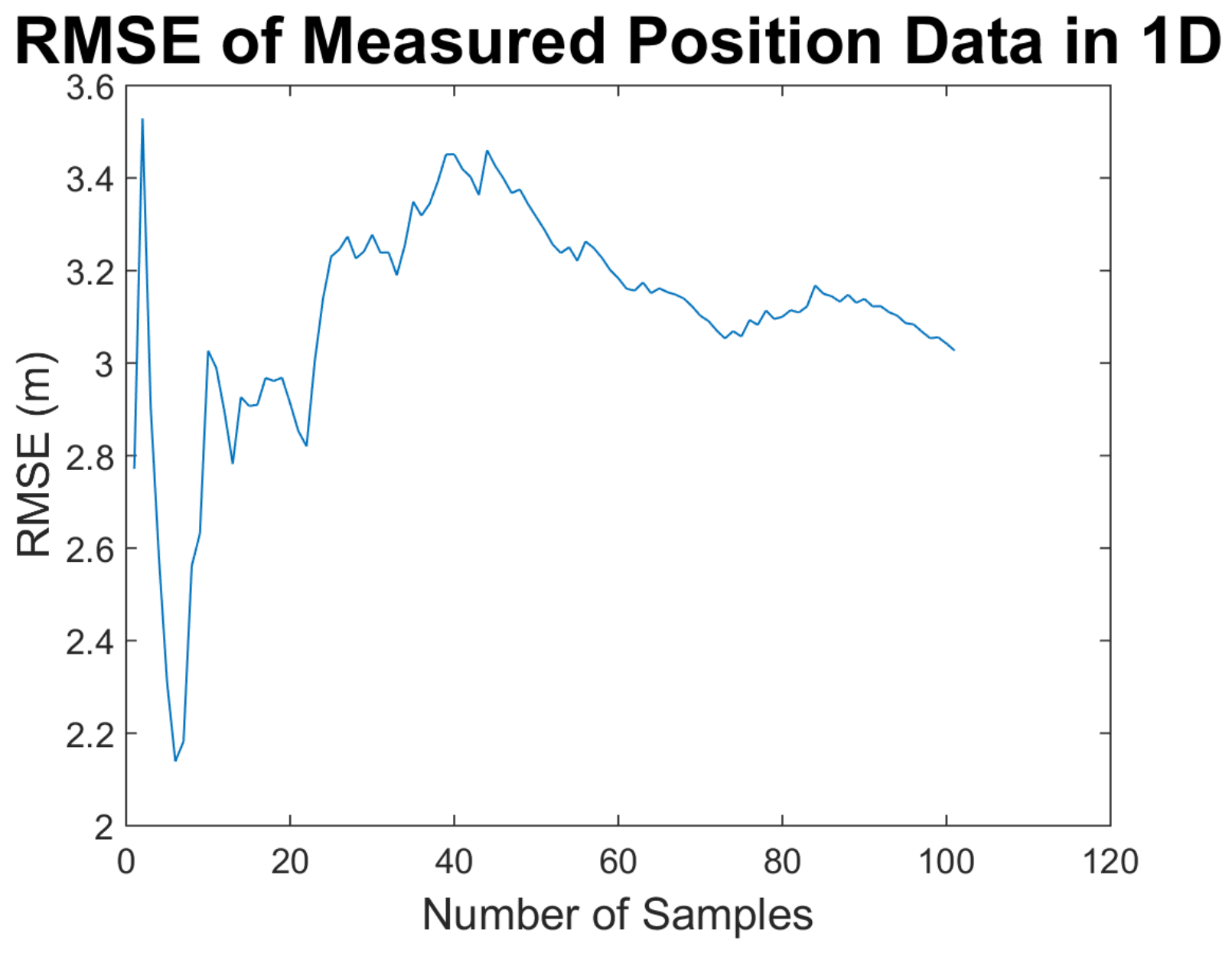

Figure 4-4 : RMSE of Measured Data Location 
Figure 4-5 demonstrates a decrease in RMSE as the sample size increases and is relatively lower in magnitude compared to Figure 4-4. This demonstrates that there is an enhancement in location data of the target. Thus in turn the velocity measurement of the target can be predicted as the sample time is known and the position data between two samples are closer to true value. Thus RMSE of the predicted velocity can be seen to improve from Figure 4-5.

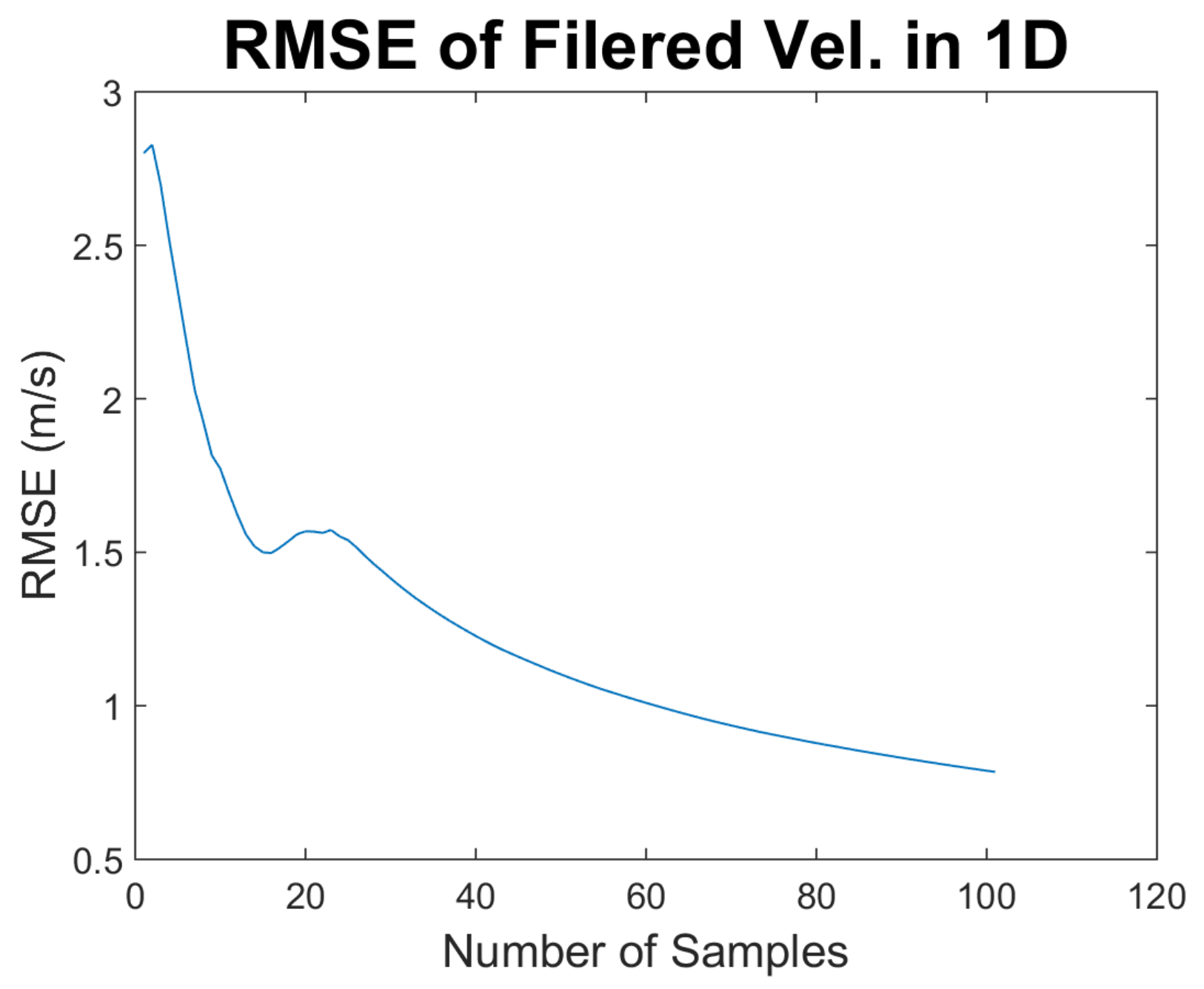

Figure 4-5 : RMSE of velocity prediction from the KF 
The output from the KF for velocity is presented in Figure 4-6 where the KF estimate is approaching the true velocity measurement as time progresses.

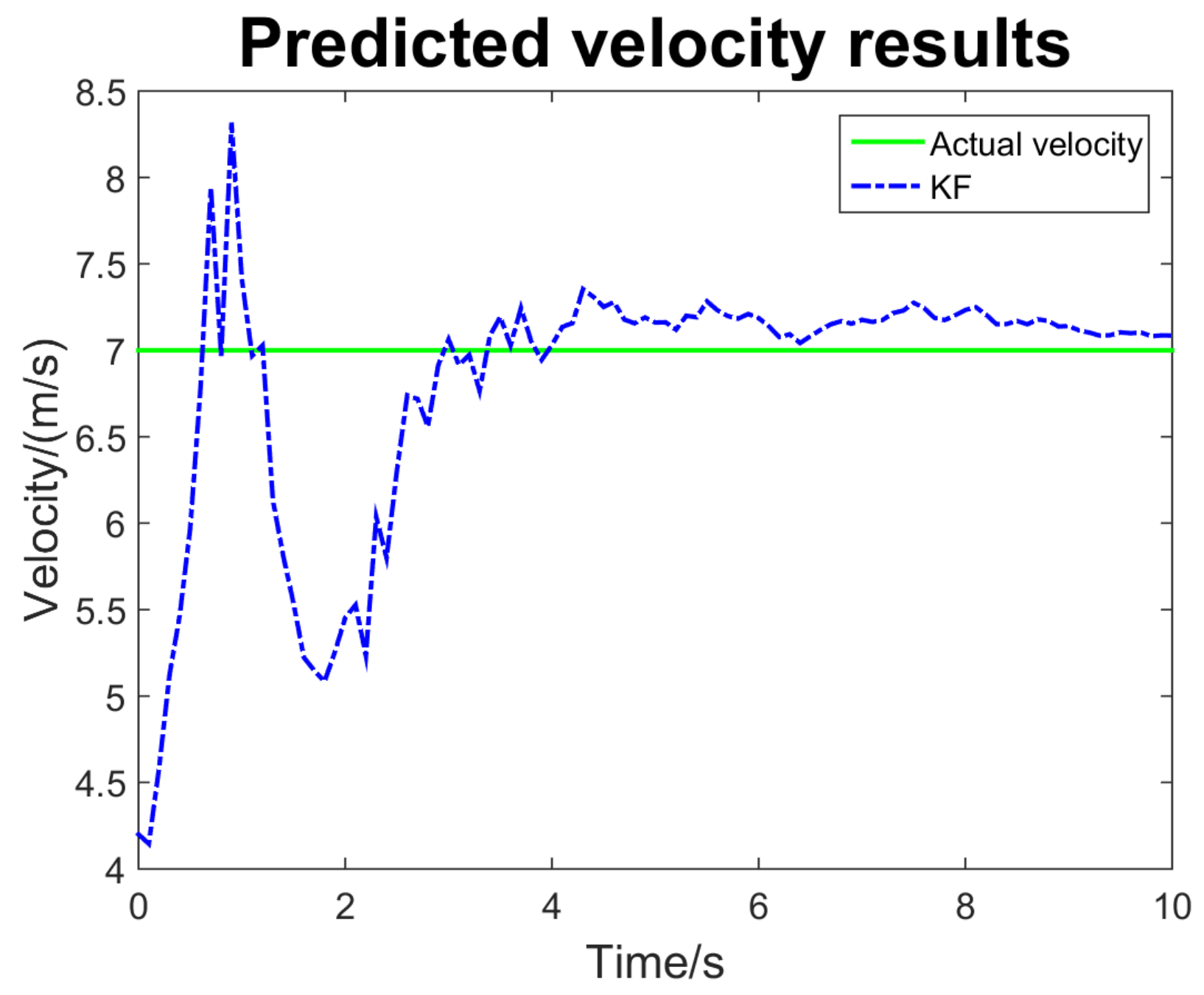

Figure 4-6 : Velocity output from KF

In the next chapter there will be demonstrations of similar graphs but this time with measurements taken from multilateration techniques that include TDOA that has no velocity dependence and FDOA. 


\subsection{Chapter Summary}

In this chapter, we covered sources of noise when using the multilateration technique.

Then covered CRLB, which provides a lower bound on variance of any unbiased

estimator, thus providing a benchmark for TDOA and FDOA measurements. This will be used for the purpose of computing noise for our simulation model. We then covered Kalman Filtering derivation in one dimension and finally illustrated how it can be used with a target maintaining constant velocity. 


\section{Chapter 5 Radar Simulation on Matlab}

The simulation results for the location of a moving target with constant velocity where prior knowledge of both the location and velocity are unknown will be covered in this chapter. The aim of the experiment is to utilize three receivers initially in this multistatic radar setup to get a fairly accurate velocity measurement. This will be done using the TDOA multilateration technique. Next, the experiment will utilize two receivers to geolocate and track the target while the multistatic radar setup maintains good GDOP. The second half of the experiment will use both the TDOA and FDOA multilateration techniques to geolocate the target. The modified HT will be used to gather location measurements by fusing multilateration measurements. The KF will be used to filter the measurements from the modified HT and track the target.

\subsection{Hybrid Geolocation and Tracking Experiment Setup}

I will outline the constant parameters to be used for the experiments in the following sections. Next I will outline the experimental model that encompasses the dual stage method where data gathered by three receiver antennas is reduced down to two receiver antennas.

\subsubsection{Parameter and Noise}

The parameters in table 5-1 are going to be used in all experiments performed in the subsequent sections. The experiment will be performed at the UHF band that can make use of wireless cellular transmitters readily available to operate in passive mode. The 
standard deviations for range will be used for TDOA measurements using Gaussian noise. The frequency standard deviation will be used for FDOA measurements using Gaussian noise.

Table 5-1 : Constant Experiment Parameters

\begin{tabular}{|c|c|}
\hline PRI (s) & $120 \mathrm{e}-6$ \\
\hline Pulse Width - Pw (s) & $50 \mathrm{e}-6$ \\
\hline Operating Frequency - f0 (Hz) & $500 \mathrm{e} 6$ \\
\hline Integration Time - T (s) & 0.1 \\
\hline BW $(\mathrm{Hz})$ & $20 \mathrm{e} 3$ \\
\hline$\sigma_{\text {TIME }}(\mathrm{s})$ & $\sim 1 \mathrm{e}-7$ \\
\hline$\sigma_{\text {RANGE }}(\mathrm{m})$ & $\sim 30$ \\
\hline$\sigma_{\text {FREQUENCY }}(\mathrm{Hz})$ & $\sim 1 \mathrm{e}-3$ \\
\hline
\end{tabular}

The standard deviation for range and frequency were both computed using equations 4.6 and 4.7 with SNR of $0 \mathrm{~dB}$. The effective bandwidth $\mathrm{B}_{\mathrm{RMS}}$ and effective duration $\mathrm{T}_{\mathrm{RMS}}$ were taken to be approximately $30 \%$ of the actual signal bandwidth and integration time respectively. This is because no signal is a perfect square hence this assumption was made.

\subsubsection{Dual Stage Method}

The flow chart diagram in Figure 5-1 illustrates the dual stage method with essentially two stages. The first stage is the velocity prediction using TDOA multilateration technique only that will be covered in section 5.2 with simulation results. The next stage 
is using TDOA and FDOA multilateration techniques to geo-locate the target position. This will be covered in section 5.3.

The chart in Figure 5-1 demonstrates that we require three receiver antennas to measure two TDOA values that create lines of position. The modified Hough Transform is used to search through an area where potentially the target lies. Hence a peak will be detected where the lines of position intersect providing the next stage with a measured target location. After having collected five or more samples, we can use this to compute an initial velocity for the prediction filter stage, the Kalman Filter. By using the Kalman Filter to predict the next target location, the measurements for location between two sample points smooth out and that in return gives us a filtered target velocity that is closer to the true target velocity. Once the refined target velocity from the Kalman Filtering stage is within a reasonable margin between samples over several samples, we can assume this is the steady velocity.

Once the velocity of the target has been predicted, we can now use only two receiver antennas to measure the TDOA and the FDOA. This is important in case one of three receiver's loose connection with central base to relay synchronized signals or is taken out by the enemy. These multilateration techniques form lines of position that intersect, at the measured target location, the Modified Hough Transform provides measured target location. This is then further refined using the Kalman Filter to track the target position. 


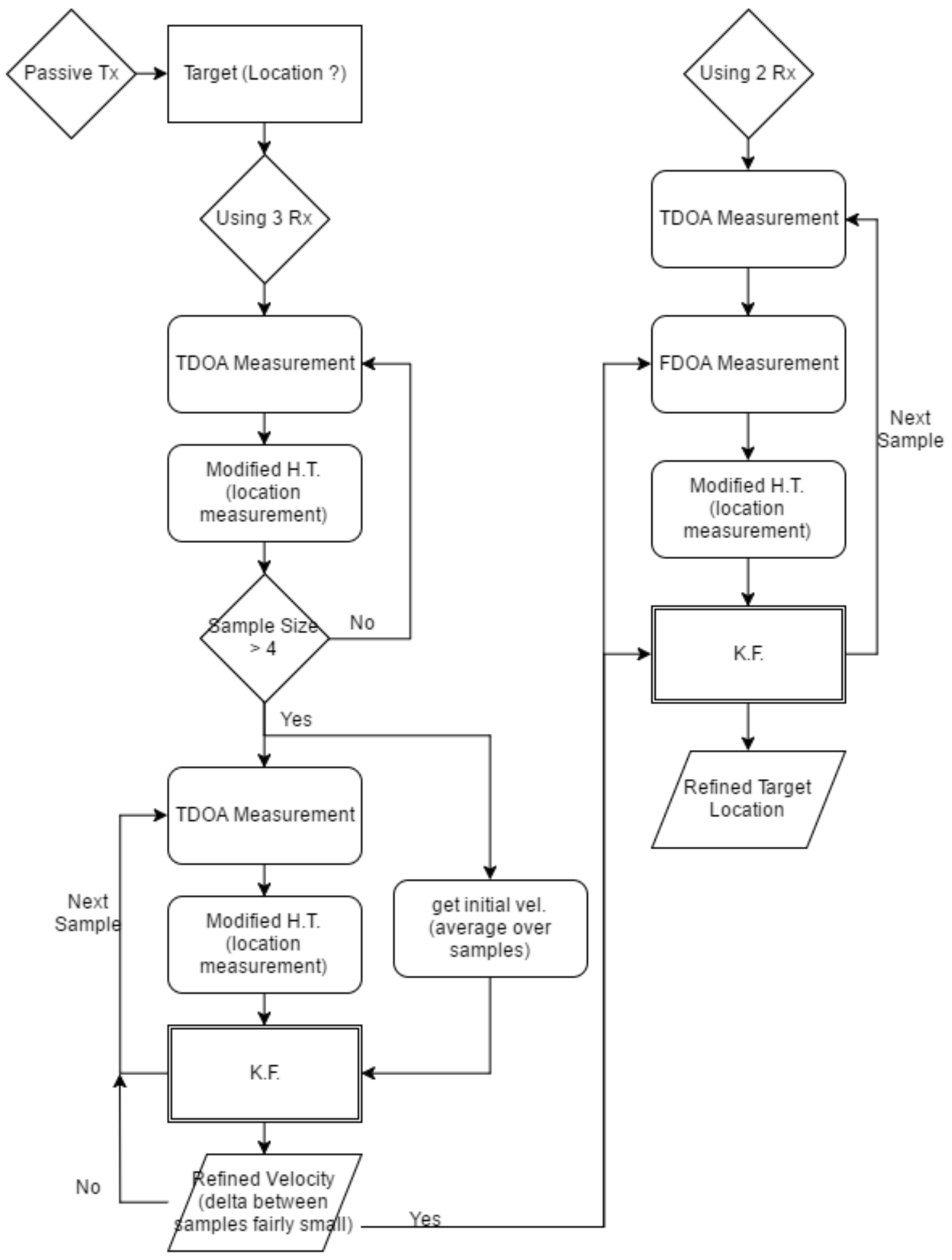

Figure 5-1 : Flow Diagram Indicating the Dual Stage Method 
As long as the target is within baseline of our multilateration radar setup, meaning we have good GDOP, the filtered target location has an improvement to the RMSE. The reason for using the dual stage method is so target tracking can continue with improvement to the RMSE of the predicted target location if one antenna loses connection with the central station or an enemy spots and destroys an antenna.

\subsection{Time Difference Model for Velocity Prediction}

The TDOA simulation will be presented in this section with graphs representing measurements in one dimension. The experiment is performed with parameters indicated in Table 5-2 with 3 receivers distributed. The target's initial position is placed outside the multistatic radar baseline to indicate the effect of GDOP on the measurements. There are 400 samples taken at 0.1 second intervals.

Table 5-2 : Parameters for Simulation using TDOA multilateration Technique

\begin{tabular}{|c|c|}
\hline Initial Target Location along X (m) & 3500 \\
\hline Initial Target Location along Y (m) & 700 \\
\hline Target Velocity X (m/s) & -120 \\
\hline Target Velocity Y (m/s) & -8 \\
\hline TX (Source of Opportunity) X, Y (m, m) & For example (0,0) \\
\hline RX1 Antenna X, Y (m, m) & $(0,0)$ \\
\hline RX2 Antenna X, Y (m, m) & $(1000,1000)$ \\
\hline RX3 Antenna X, Y (m, m) & 400 \\
\hline Sample Size N & 0.1 \\
\hline Sample Time (s) & \\
\hline
\end{tabular}


The experimental setup is indicated in Figure 5-2 with the following three receivers Rx1, $\mathrm{R} \times 2$ and $\mathrm{Rx} 3$. The target's location starts from the right top corner and ends at the bottom left. It is mapped out on a two dimensional plane with antennas Rx1 and R2 used to create one LOP and Rx1 and Rx3 used to create the second LOP.

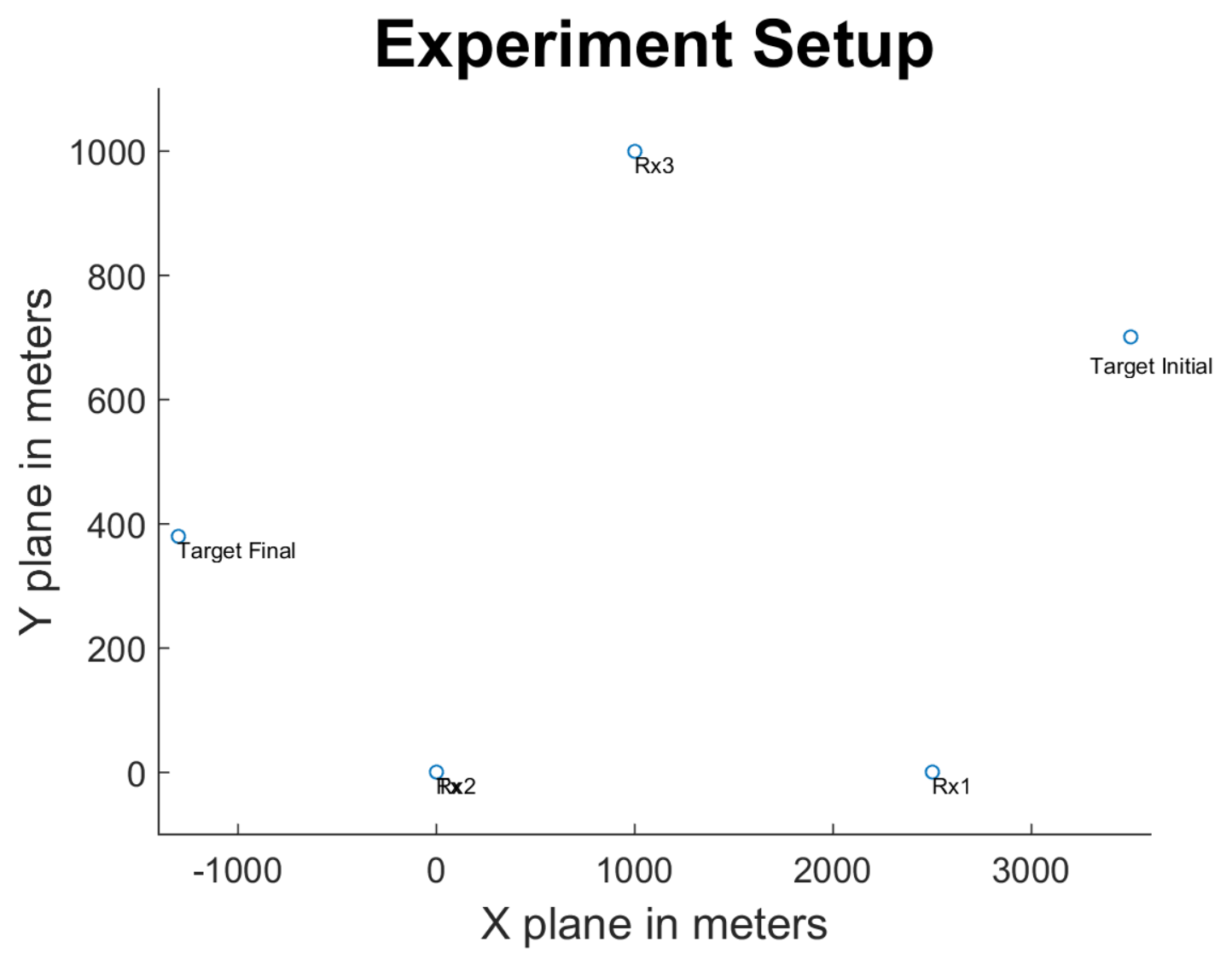

Figure 5-2 : Experiment Setup for TDOA

The geometric dilution of precision or GDOP indicates a low value as the target approaches closer to all three receivers. This indicates that we have a good GDOP thus low measurement error due to the geometry between the target and the receivers. This is represented in Figure 5-3. 
GDOP

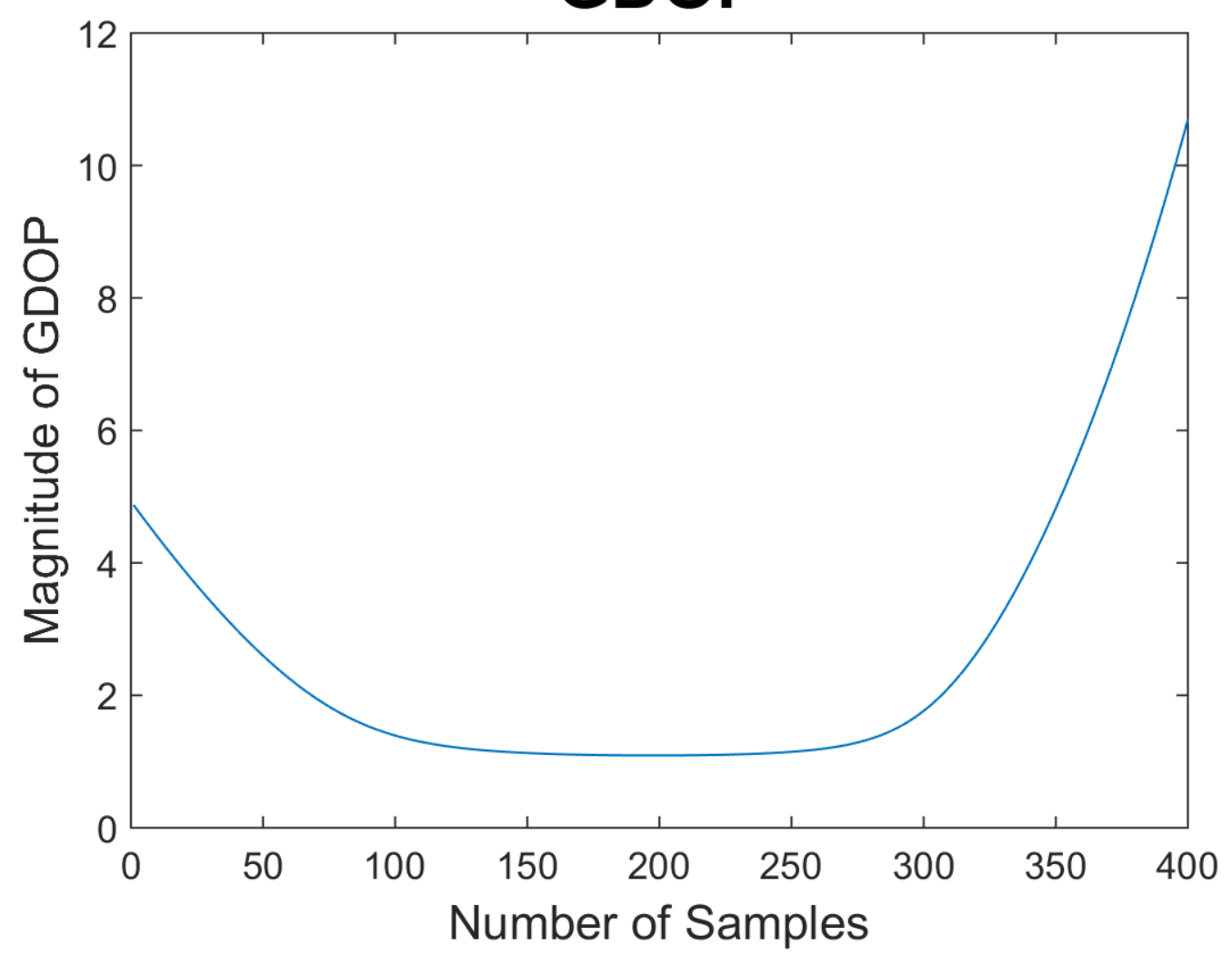

Figure 5-3: GDOP due to TDOA experiment

The target's position along the $\mathrm{x}$-axis is represented in Figure 5-4 where the actual position is a linear line that starts at 3500 meters and ends at -1300 meters. The negative measurement in distance is relative to the second receiver positioned at zero along the $\mathrm{x}$ axis. Noise in measurement is seen to reflect the GDOP curve in Figure 5-3. 


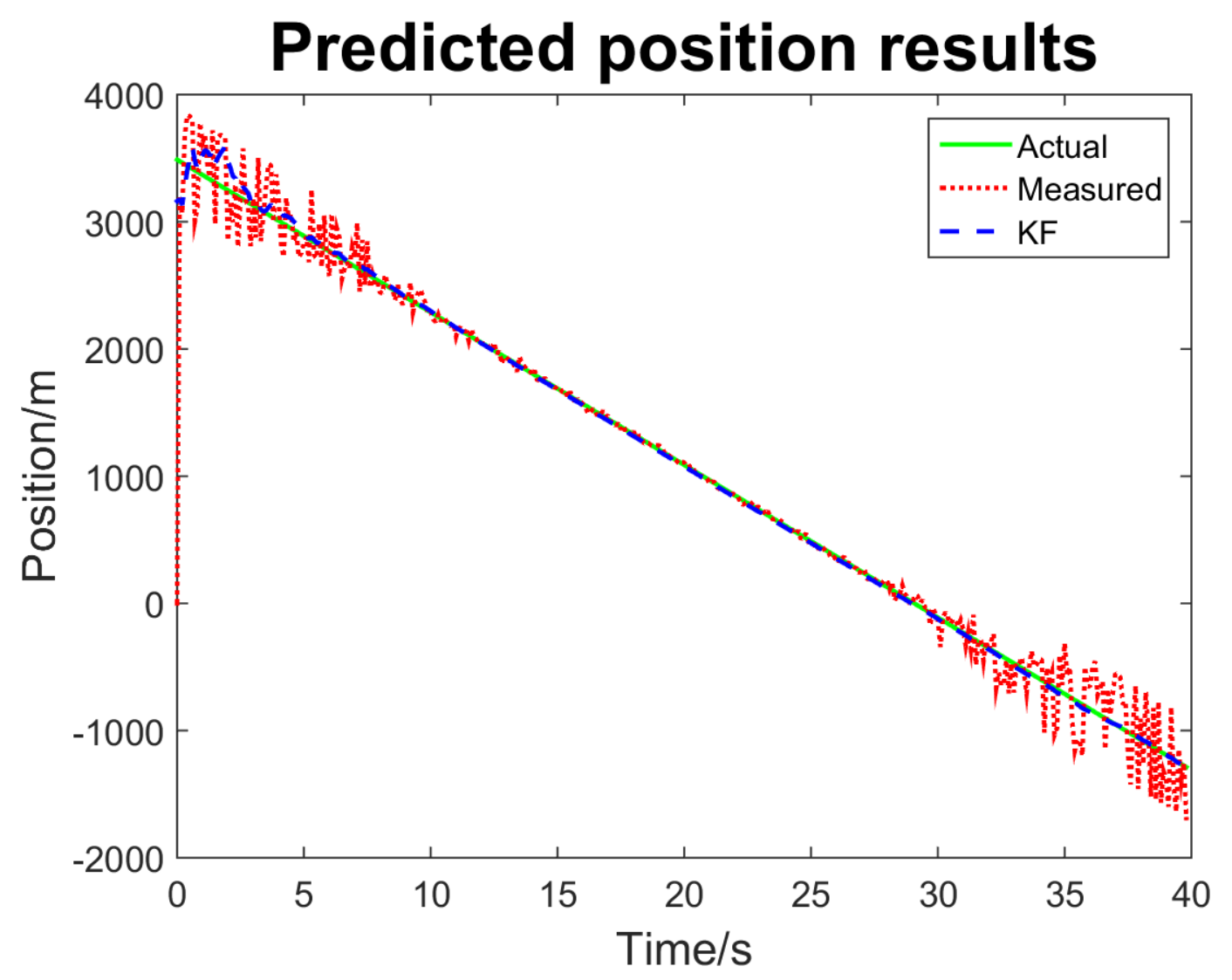

Figure 5-4 : X-axis position data for the TDOA experiment

From Figure 5-5 we can see the velocity prediction from the Kalman Filter output appears to have settled after 10 seconds thus the delta between two filtered measurements is relatively steady. This happens once we have obtained good GDOP. Between 10 seconds to 30 seconds we see relatively linear update in the velocity prediction. After 30 seconds when the GDOP is bad, we see fluctuations in the predicted velocity. 


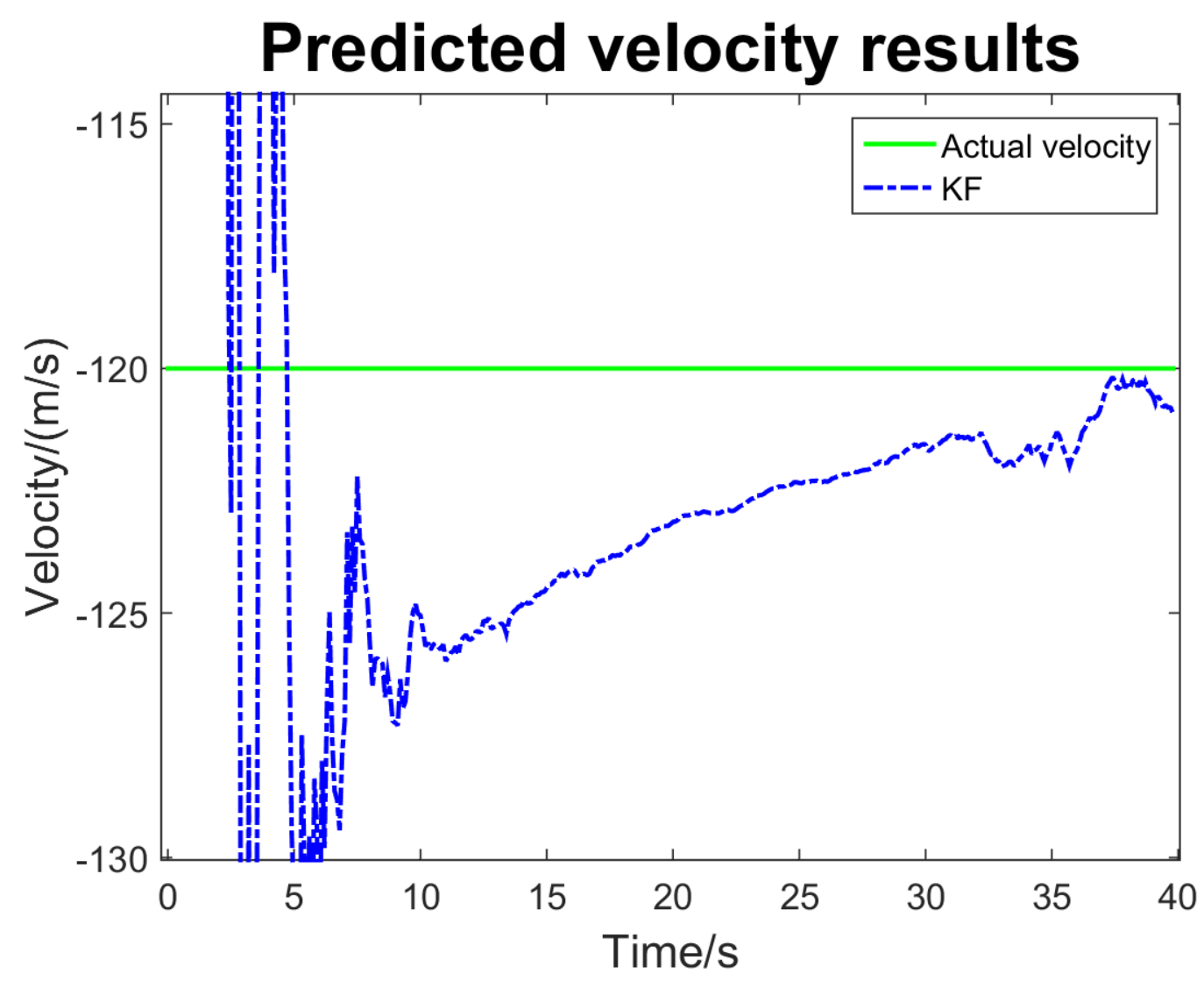

Figure 5-5 : X-axis prediction of target velocity for the TDOA experiment

Figure 5-6 represents the Root Mean Squared Error of the velocity prediction along the xaxis. It can be seen that the rate of decline in the RMSE decreases as we approach 300 samples which is when the GDOP from Figure 5-3 represents worsening GDOP. For this thesis, I am interested in velocity predictions once the difference in predicted velocity between 10 samples is less than one meter per second. 


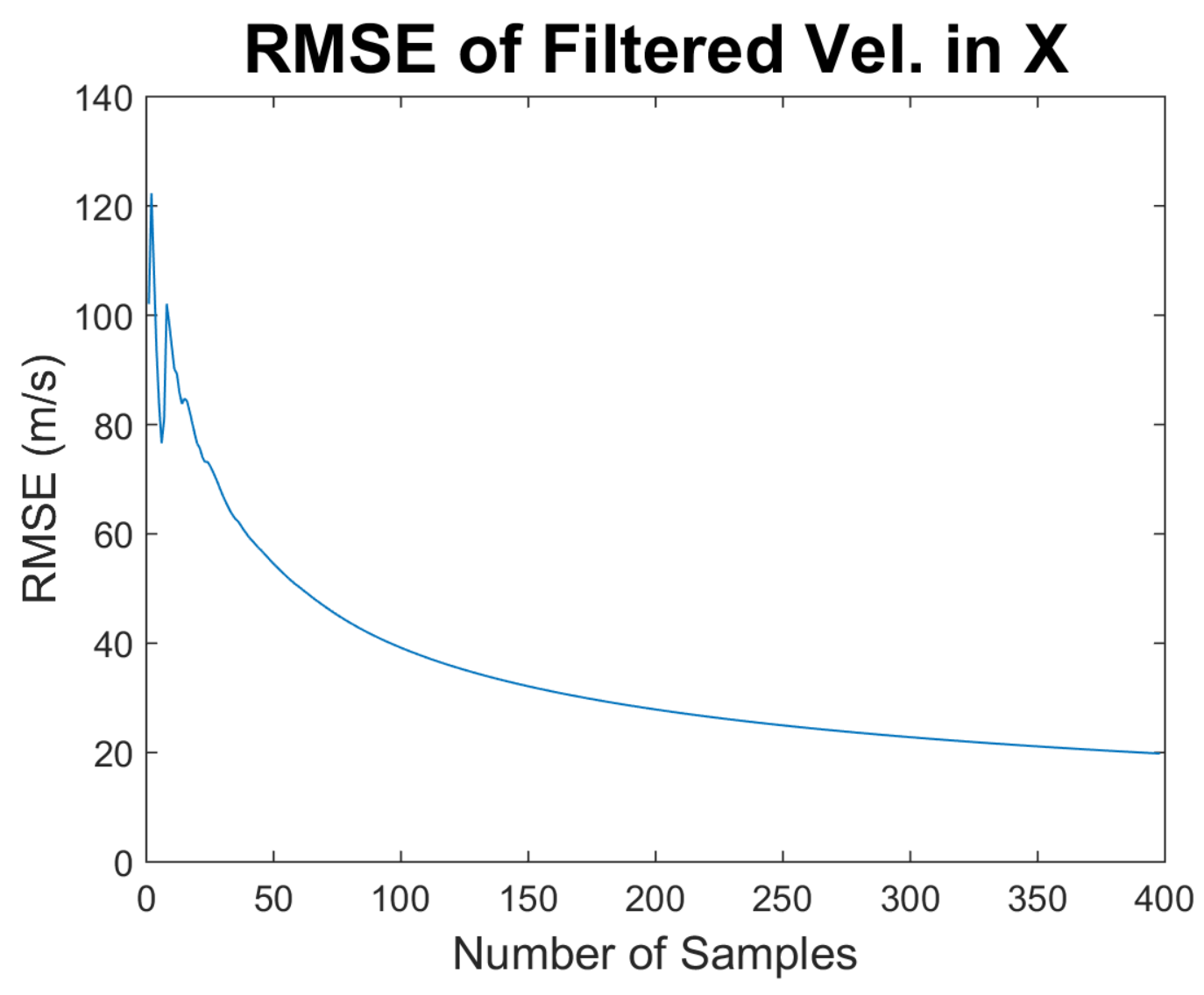

Figure 5-6 : X-axis Root Mean Squared Error for the filtered target velocity

The target's position along the y-axis is represented in Figure 5-7 where the actual position is a linear line that starts at 700 meters and ends at 380 meters. The predicted position results are represented by the Kalman Filter output with label KF in the Figure 57. The main influence of GDOP for the $y$-axis measurement is due to antennas Rx1 and $\mathrm{Rx} 3$. Referring to Figure 5-2, we see that the target remains relatively centered across the baseline created by antennas Rx1 and Rx3 while approaching closer to it we see a reduction in measured position data. This is because we are getting better GDOP at around 20 seconds. 


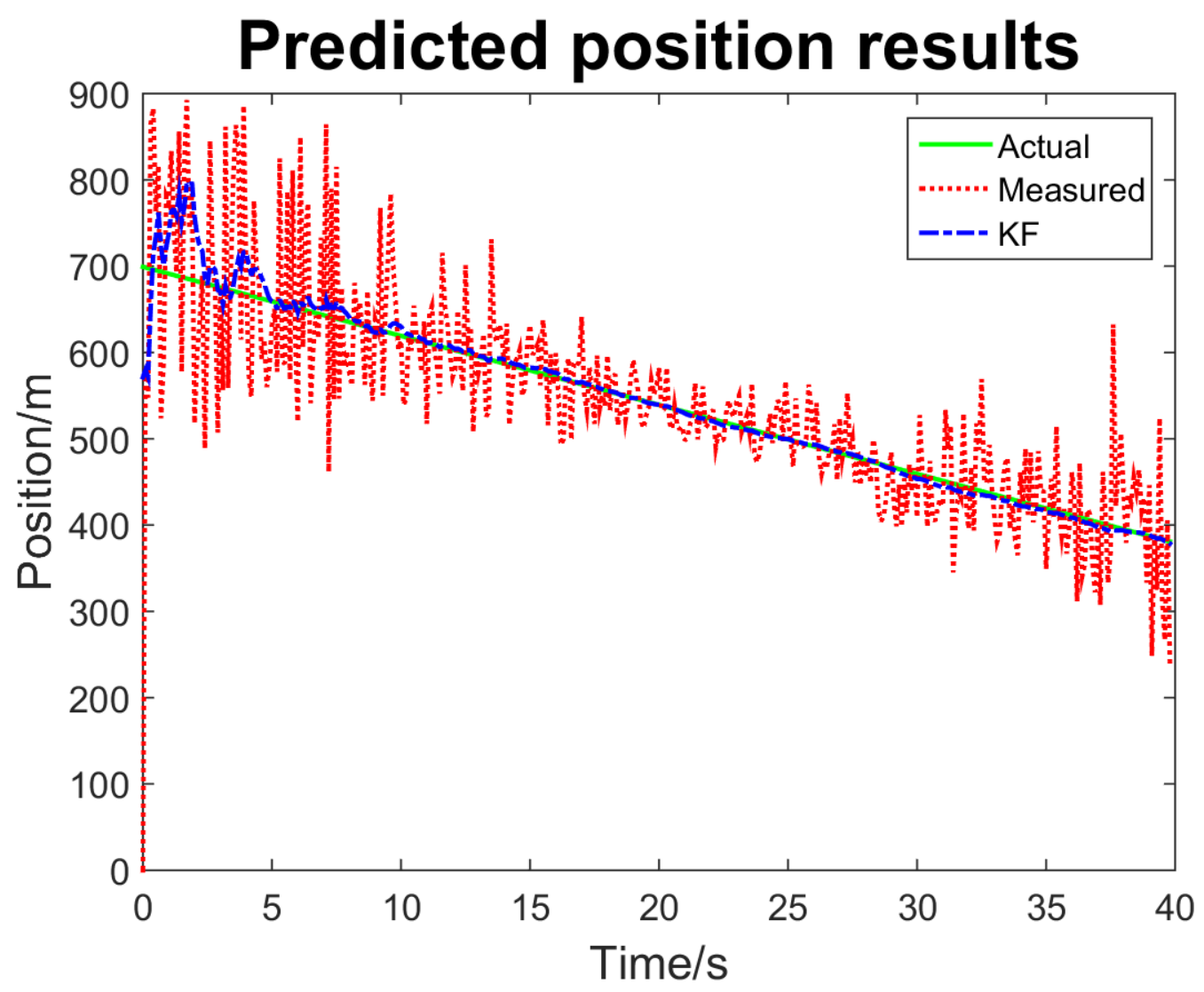

Figure 5-7 : Y-axis position data for the TDOA experiment

The y-axis velocity prediction in Figure 5-8 appears to approach the true value with a smaller error margin as compared to the $\mathrm{x}$-axis prediction in Figure 5-5. This is because the target is within the radar baseline created by antennas $\mathrm{Rx} 1$ and $\mathrm{Rx} 3$. 


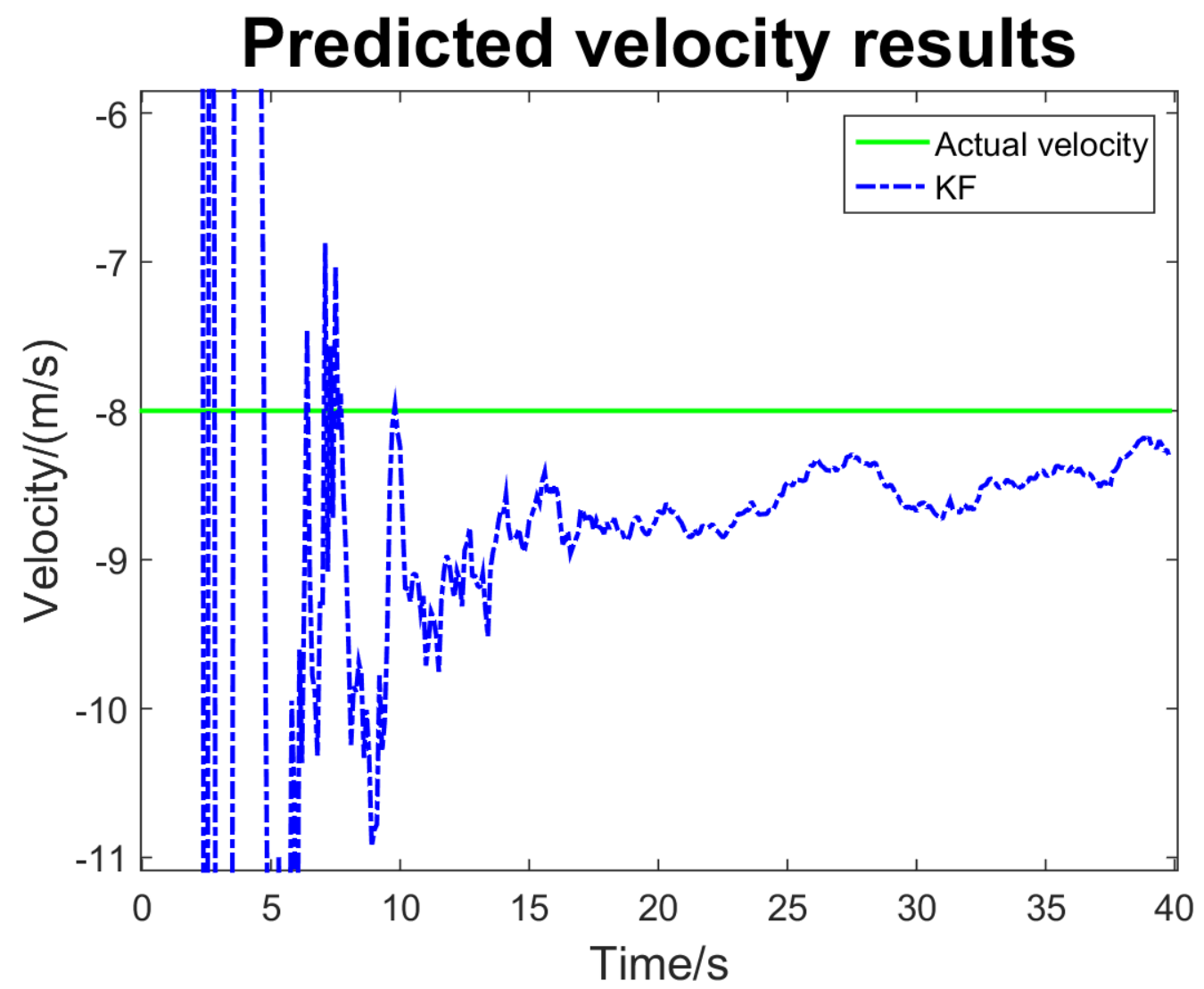

Figure 5-8: Y-axis prediction of target velocity for the TDOA experiment

The RMSE for y-axis velocity prediction, as seen in Figure 5-9, appears to flatten out at around the same value of around 20 meters per second RMSE. However, one has to look at the rate of decline which is higher for the y-axis case; this is because the target is already within the radar baseline. 


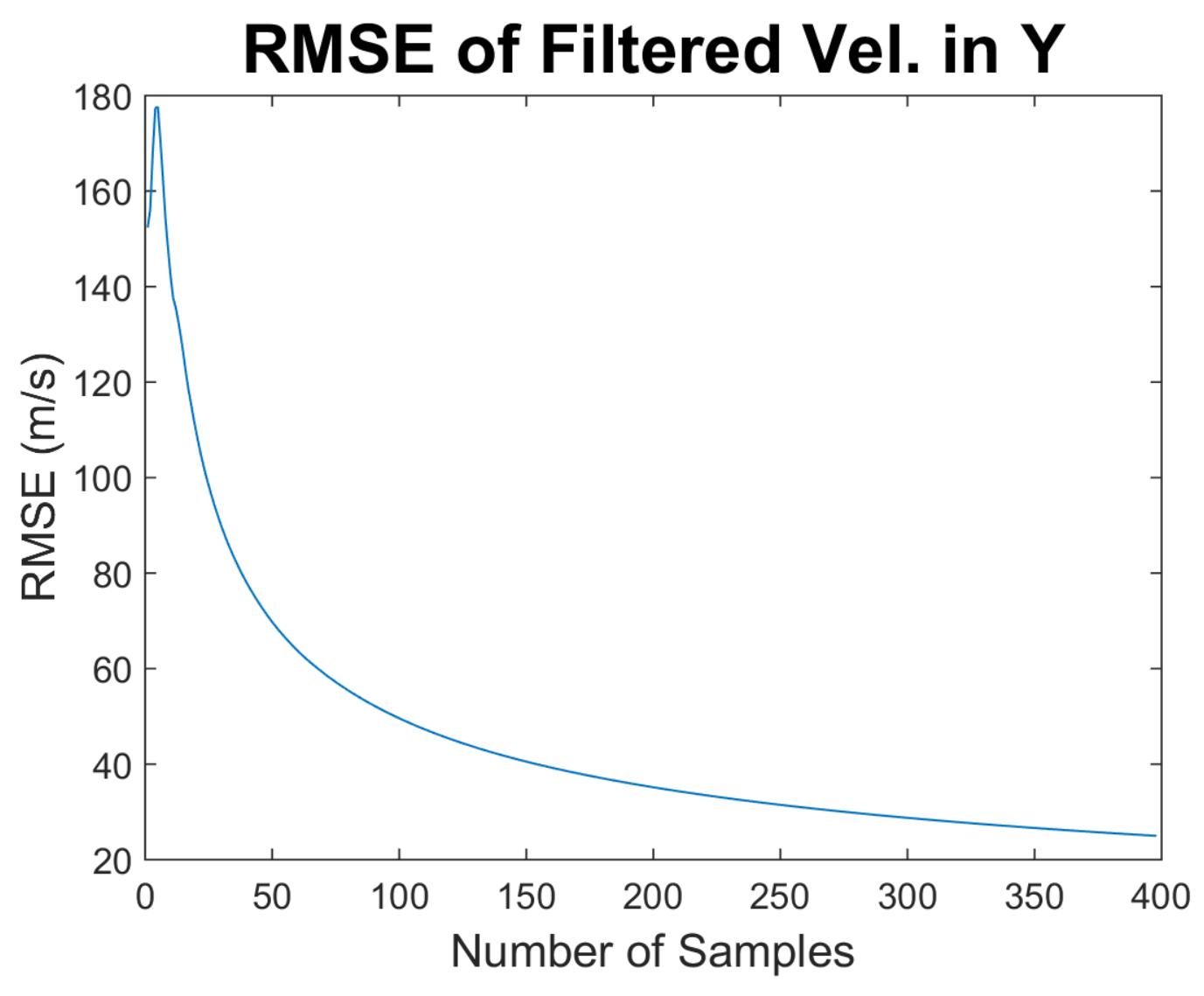

Figure 5-9 : Y-axis Root Mean Squared Error for the filtered target velocity

The velocity predictions in this section that appear at around 125 samples will be used as part of the FDOA multilateration technique. This will allow for one less antenna use. This will come in handy if an antenna is lost and tracking of the target location can continue with the remaining two antennas while continuing to maintain good GDOP.

\subsection{Application of TDOA and FDOA for Target Location}

The TDOA and FDOA simulation will be presented in this section with graphs representing measurements in one dimension. The experiment is performed with parameters indicated in Table 5-3 with 2 receivers distributed. The receivers $\mathrm{Rx} 1$ and 
$\mathrm{Rx} 2$ that remain have the same location as the experiment performed in the previous section with TDOA measurements only. The target's initial position is placed at the center of the multistatic radar baseline. This is to simulate the fact that the velocity prediction has been obtained and target tracking will now be performed with TDOA and FDOA. There are 400 samples taken at 0.1 second intervals in this experiment.

Table 5-3 : Parameters for Simulation using TDOA and FDOA multilateration Techniques

\begin{tabular}{|c|c|}
\hline Initial Target Location along X (m) & 1250 \\
\hline Initial Target Location along Y (m) & 550 \\
\hline Target Velocity X (m/s) & -120 \\
\hline Target Velocity Y (m/s) & -8 \\
\hline TX (Source of Opportunity) X, Y (m, m) & For example $(0,0)$ \\
\hline RX1 Antenna X, Y (m, m) & $(2500,0)$ \\
\hline RX2 Antenna X, Y (m, m) & $(0,0)$ \\
\hline Sample Size N & 400 \\
\hline Sample Time (s) & 0.1 \\
\hline
\end{tabular}

The experimental setup is indicated in Figure 5-10 with the two receivers indicated Rx1 and $\mathrm{R} \times 2$. The targets initial location starts from the right top corner and ends at the bottom left following the same trajectory as the simulation in section 5.2. It is mapped out on a two dimensional plane with antennas Rx1 and R2 used to create one LOP for TDOA and FDOA that intersect orthogonal when we have good GDOP. 


\section{Experiment Setup}

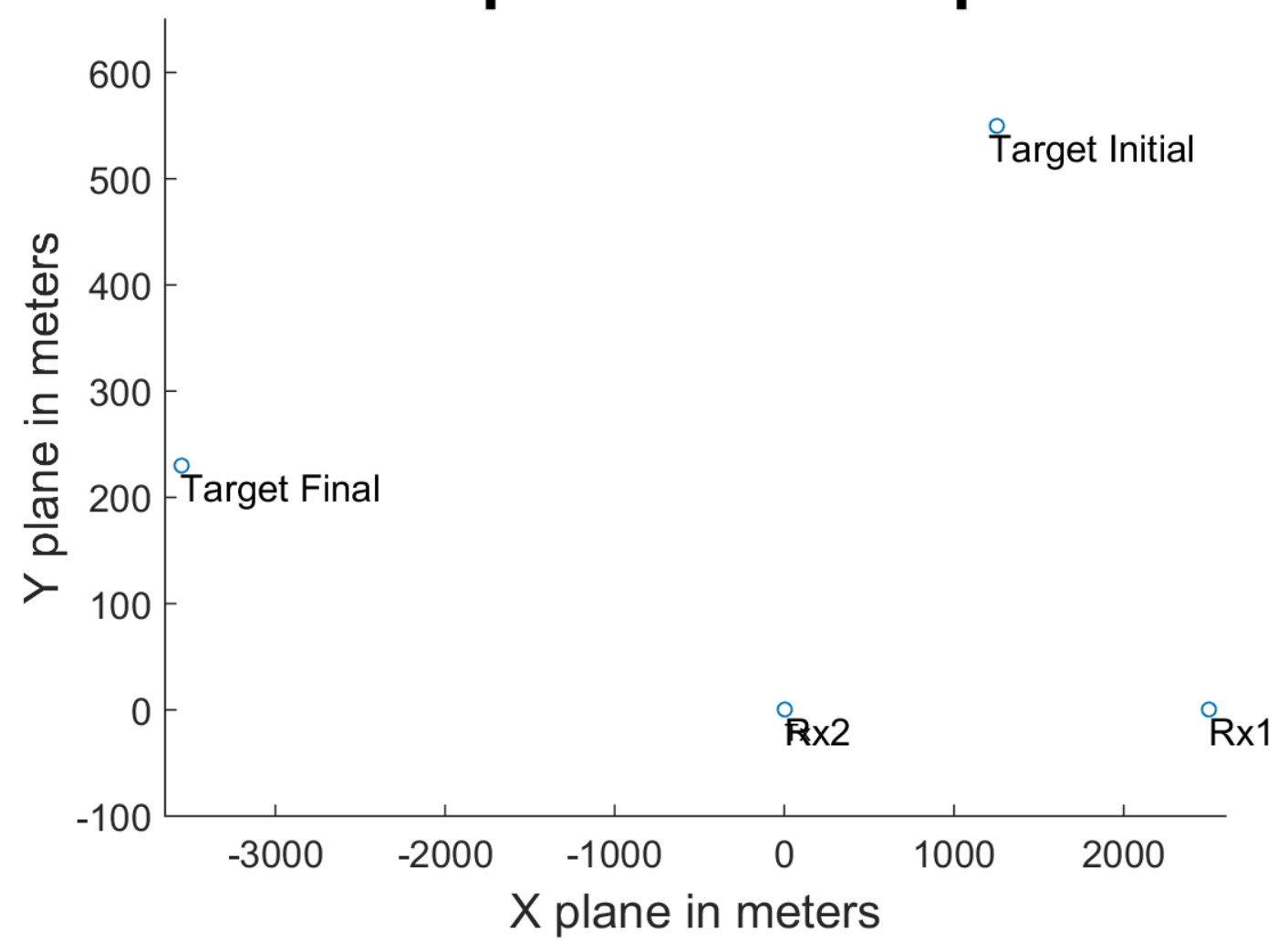

Figure 5-10 : Experiment Setup for TDOA and FDOA

The GDOP from Figure 5-11 indicates that we have a good GDOP to begin with and as the target leaves the radar baseline at around 150 samples, we start to see GDOP values going up which indicates poor GDOP. 


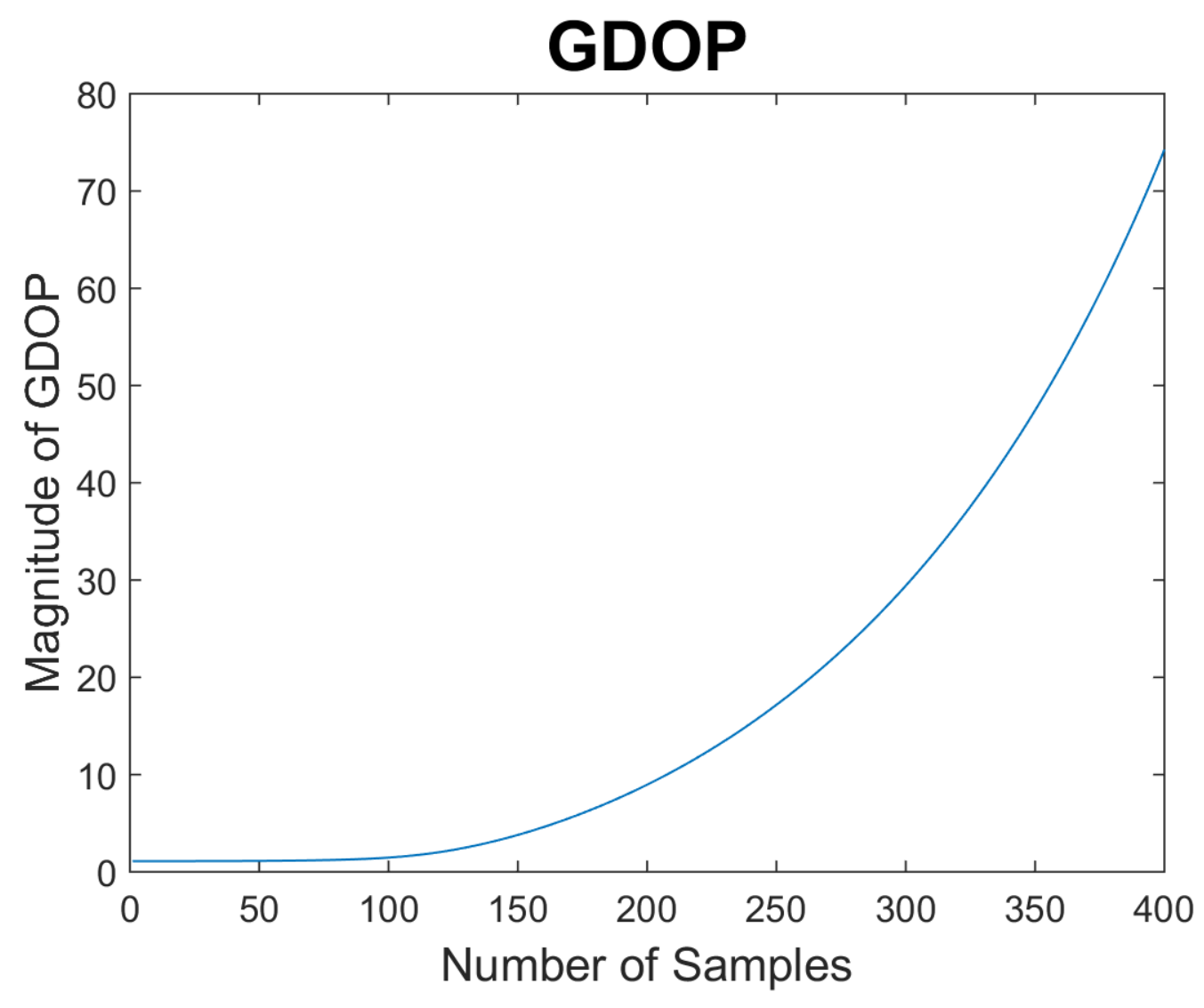

Figure 5-11 : GDOP due to TDOA and FDOA experiment

The target's position along the $\mathrm{x}$-axis is represented in Figure 5-12 where the actual position is a linear line that starts at 1250 meters and ends at -3550 meters. The negative measurement in distance is relative to the second receiver positioned at zero along the $\mathrm{x}$ axis. Noise in measurement is seen to reflect the GDOP curve in Figure 5-11. One can also observe that the measurements are being clipped at around 20 seconds. This is due to worsening GDOP that creates large noise swings. Because my modified Hough Transform scan range bins were not large enough, we see the saturation. As this is out of the scope of this thesis, hence it will not be elaborated upon. 


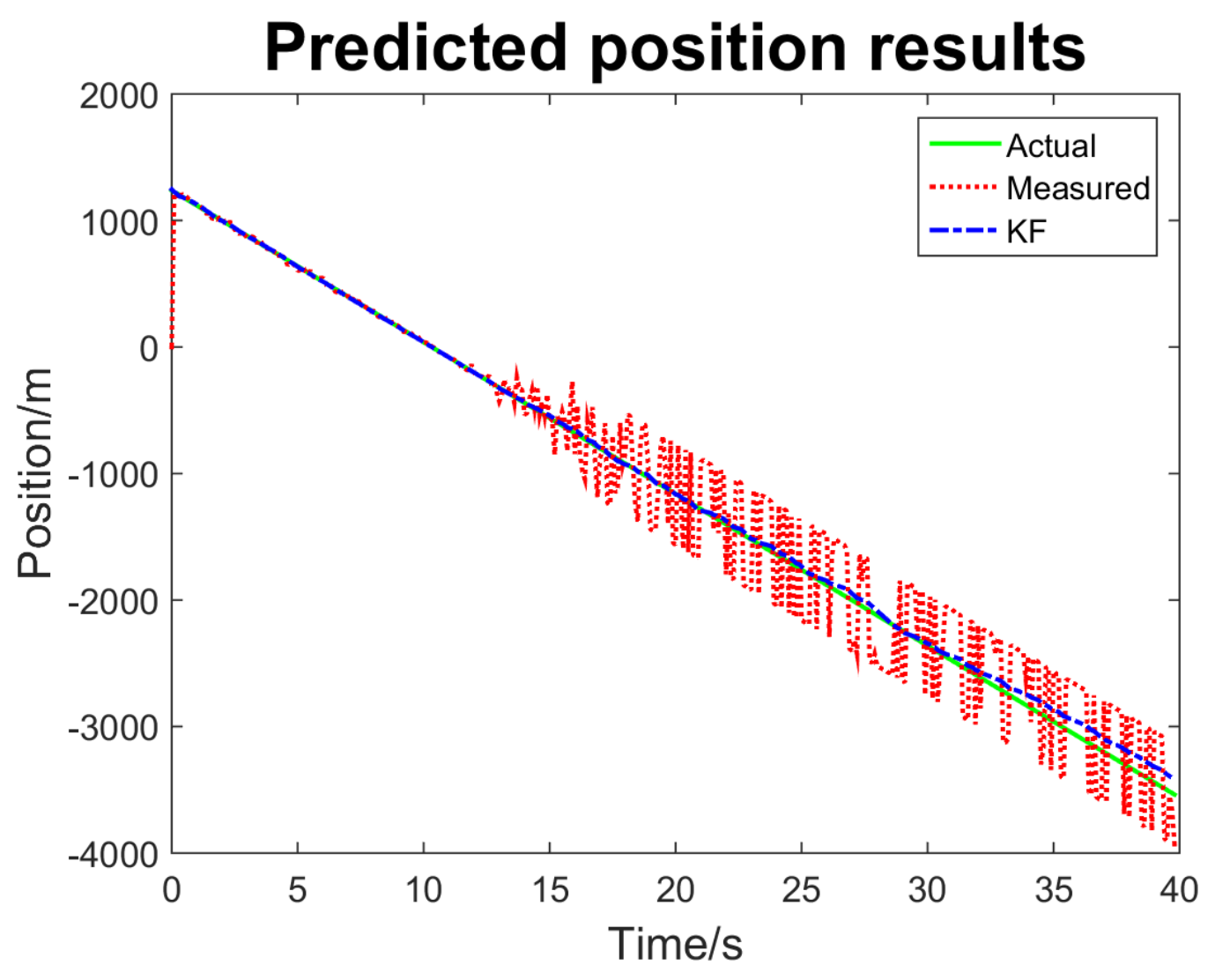

Figure 5-12:X-axis position data for the TDOA and FDOA experiment

Figure 5-13 represents the Root Mean Squared Error of the target location prediction along the X-axis. We can see the RMSE increase as the GDOP gets worse after 150 samples or when the target is at $\mathrm{x}$-axis location -500 meter approximately. 


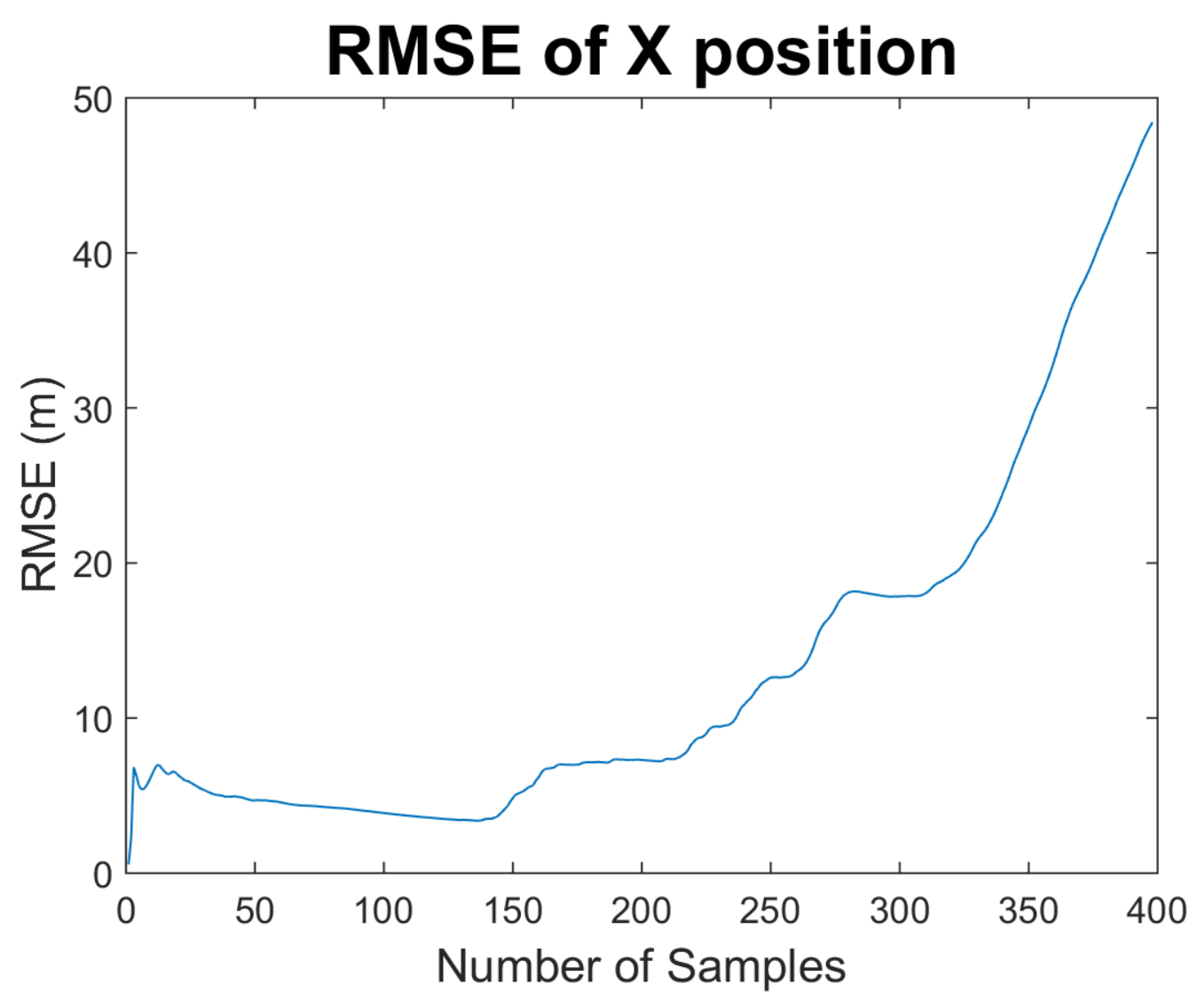

Figure 5-13 : X-axis Root Mean Squared Error for the filtered target location

The target's position along the y-axis is represented in Figure 5-14 where the actual position is a linear line that starts at 550 meters and ends at 230 meters. Noise in measurement is seen to reflect the GDOP curve in Figure 5-11. However there seems to be more noise in samples under 10 seconds. One has to note, the y-axis measurements are mainly influenced due to FDOA multilateration technique in this case due to geometry of the experimental setup. 


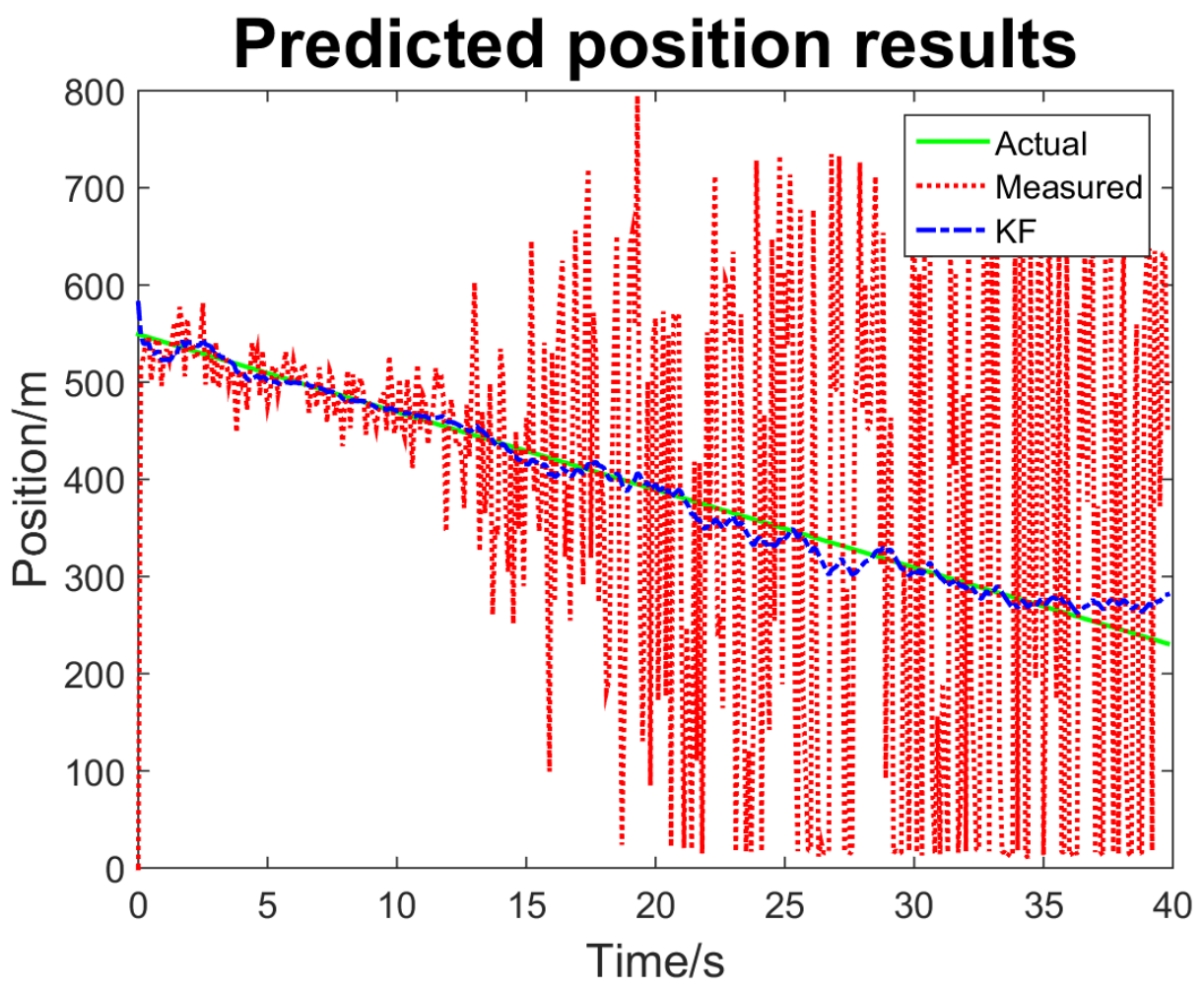

Figure 5-14 : Y-axis position data for the TDOA and FDOA experiment

The reason for the RMSE in Figure 5-15 to start higher and end higher along the y-axis is not only because of GDOP, but it is also due to the effect of the measured velocity that adds noise to the FDOA measurements along the y-axis. 


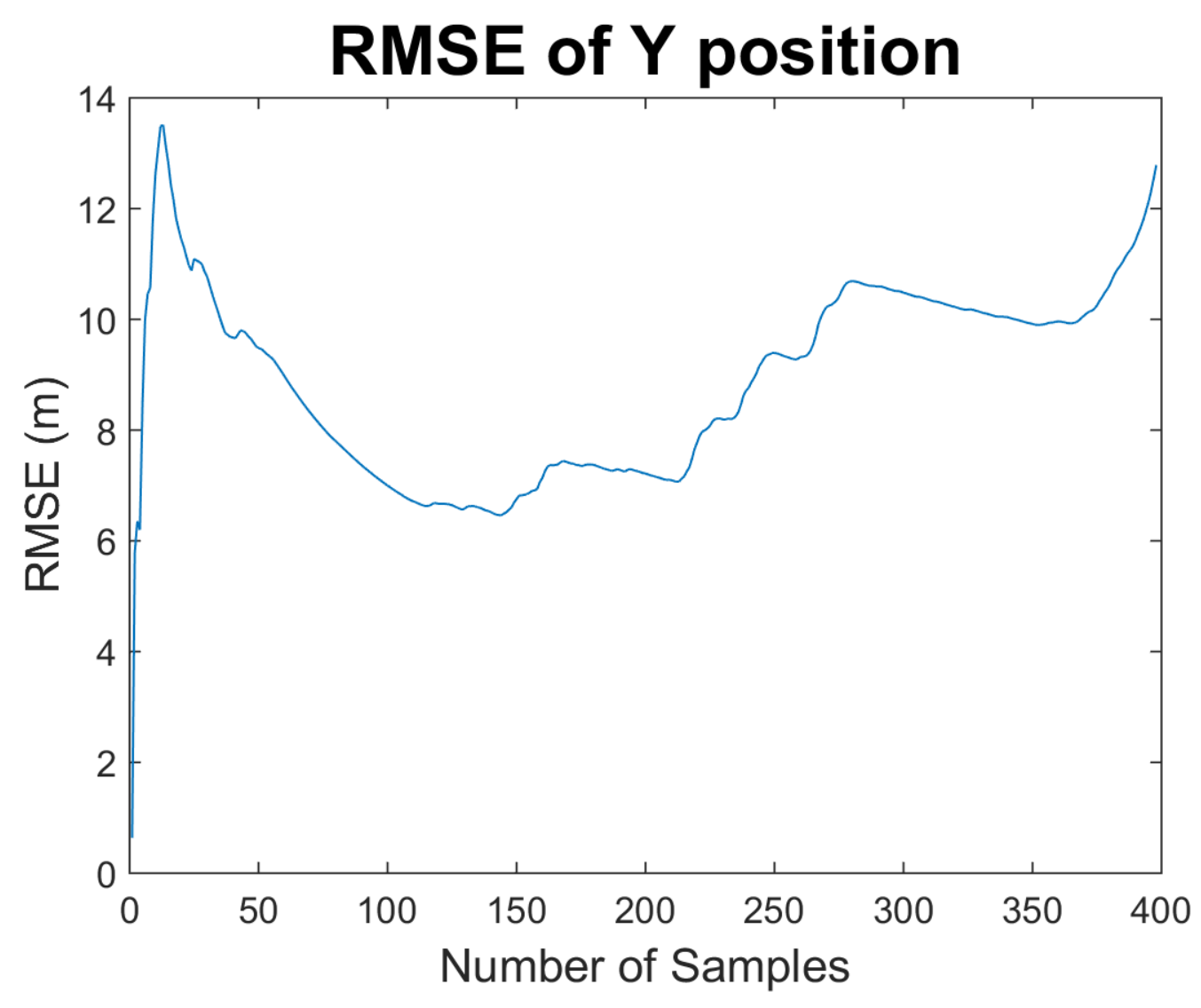

Figure 5-15 : Y-axis Root Mean Squared Error for the filtered target location

In the next section we will analyze the effects of noise in comparison to simulations performed in this section.

\subsection{Analysis of Noise}

In this section we are going to analyze the simulation output when we increase the TDOA and FDOA noise and repeat the simulation as in section 5.3. Next, we will comment on a simulation output where we used fixed TDOA and FDOA noise, hence having no Gaussian distribution and observed the measurement variation. 


\subsubsection{TDOA and FDOA with higher noise}

Figure 5-16 represents the experiment setup with target location predicted along y-axis that is represented with the line labeled KF. The experiment has all the same parameters as in section 5.3 with the exception that the standard deviation for range $\sigma_{\text {RANGE }}$ is 120 meters and standard deviation for frequency $\sigma_{\mathrm{FREQUENCY}}$ is 4 milliHertz, that is they are 4 times the value presented in Table 5-1.

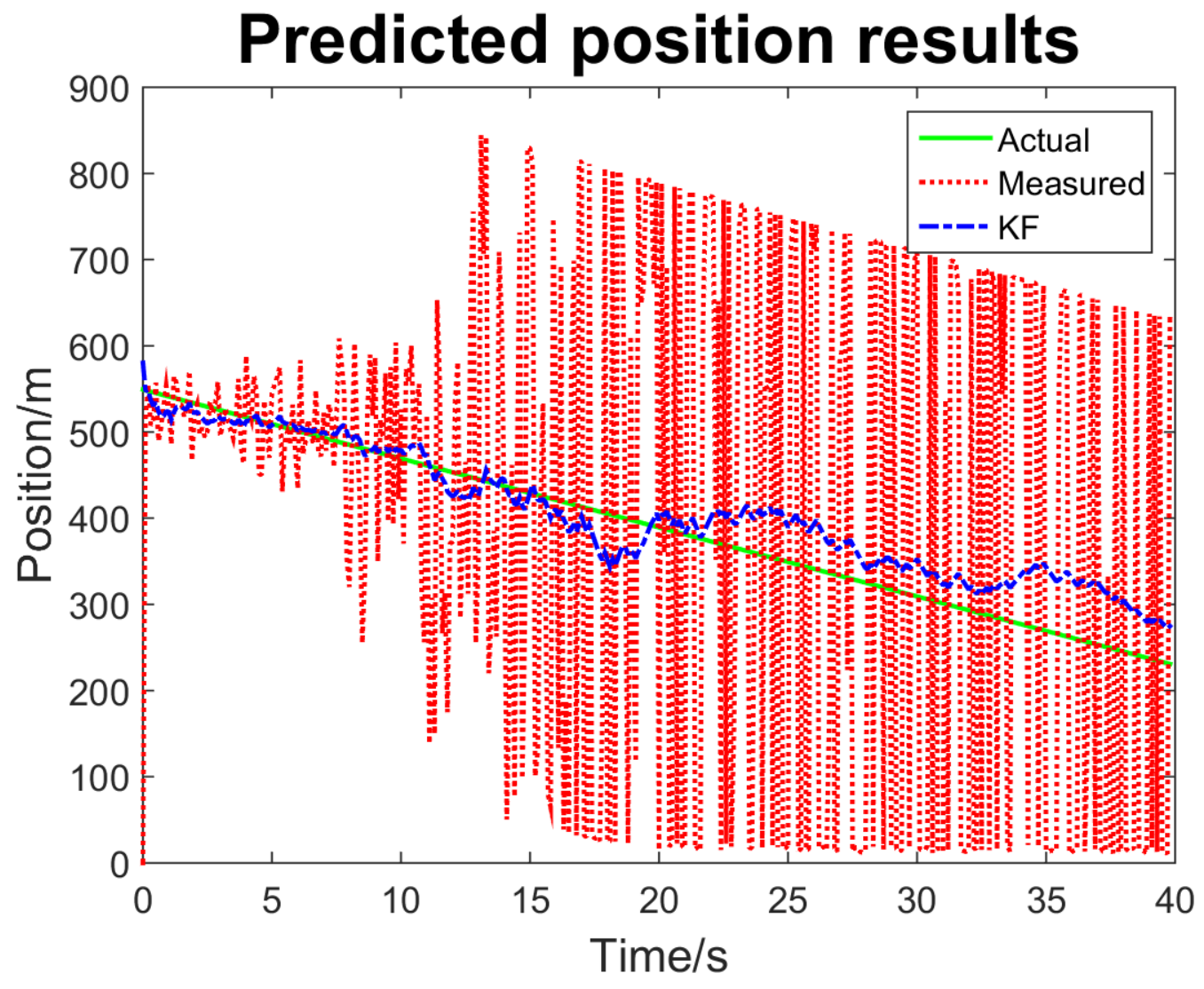

Figure 5-16: Y-axis position data for the TDOA and FDOA experiment with higher noise

From Figure 5-17 we see the RMSE result for the predicted position along the y-axis has larger error in comparison to Figure 5-15. This is due to the fact that the errors in TDOA 
and FDOA were quadrupled. This reinforces the fact that with larger noise measurements in TDOA and FDOA will affect the target location predictions with higher error.

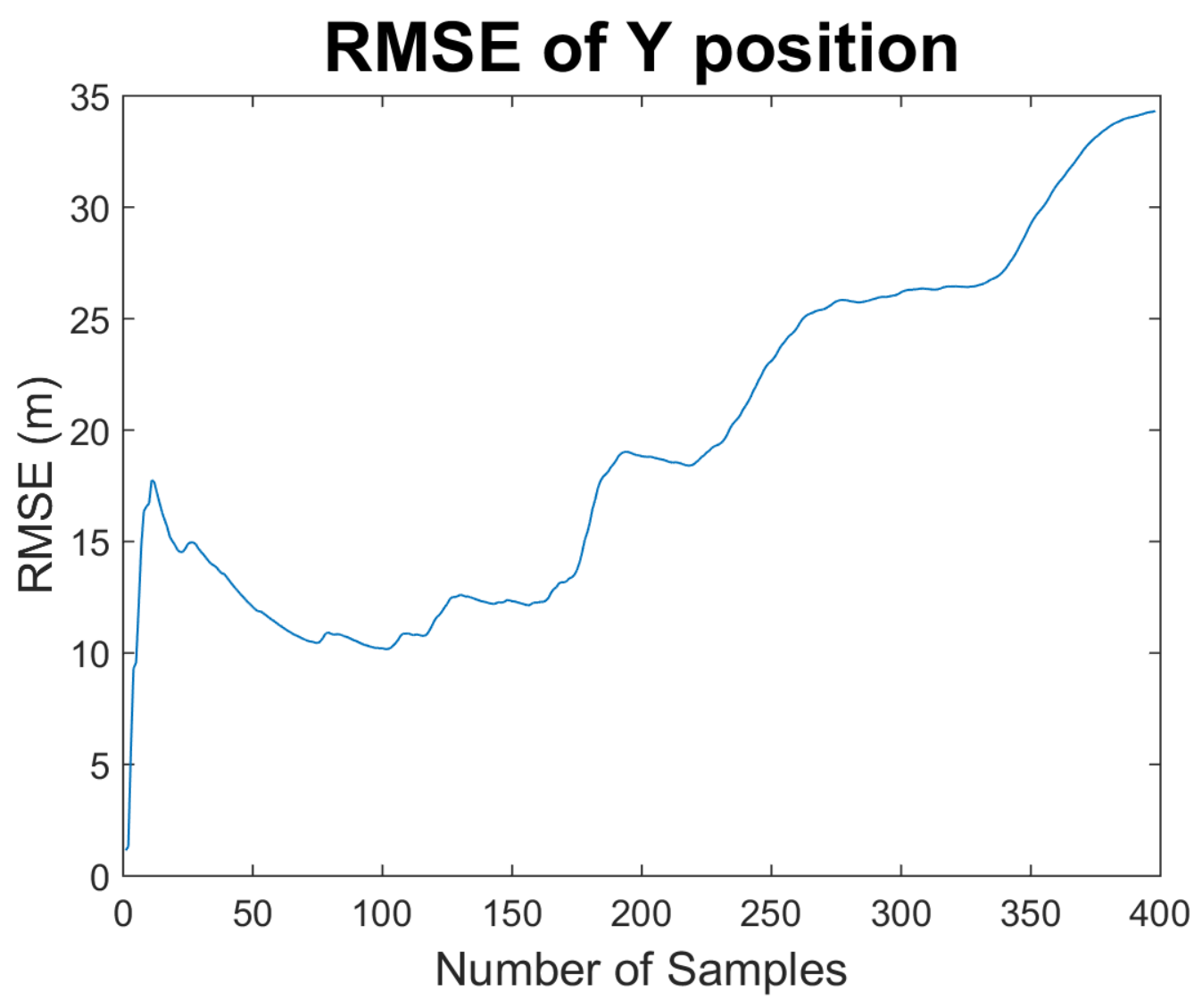

Figure 5-17 : Y-axis Root Mean Squared Error for the filtered target location with higher noise

\subsubsection{TDOA and FDAO with fixed noise}

By adding fixed noise (does not change over samples) to all measurements, we can see how GDOP affects the measurement results. In this experiment we do not use Gaussian distribution on the TDOA and FDOA noise, hence keeping it fixed. The experiment layout as seen in Figure 5-18 is similar to that in section 5.2 but with the third receiver 
not present as we are using TDOA and FDOA measurements. This setup was chosen to demonstrate the transition of GDOP from poor to good and back to poor.

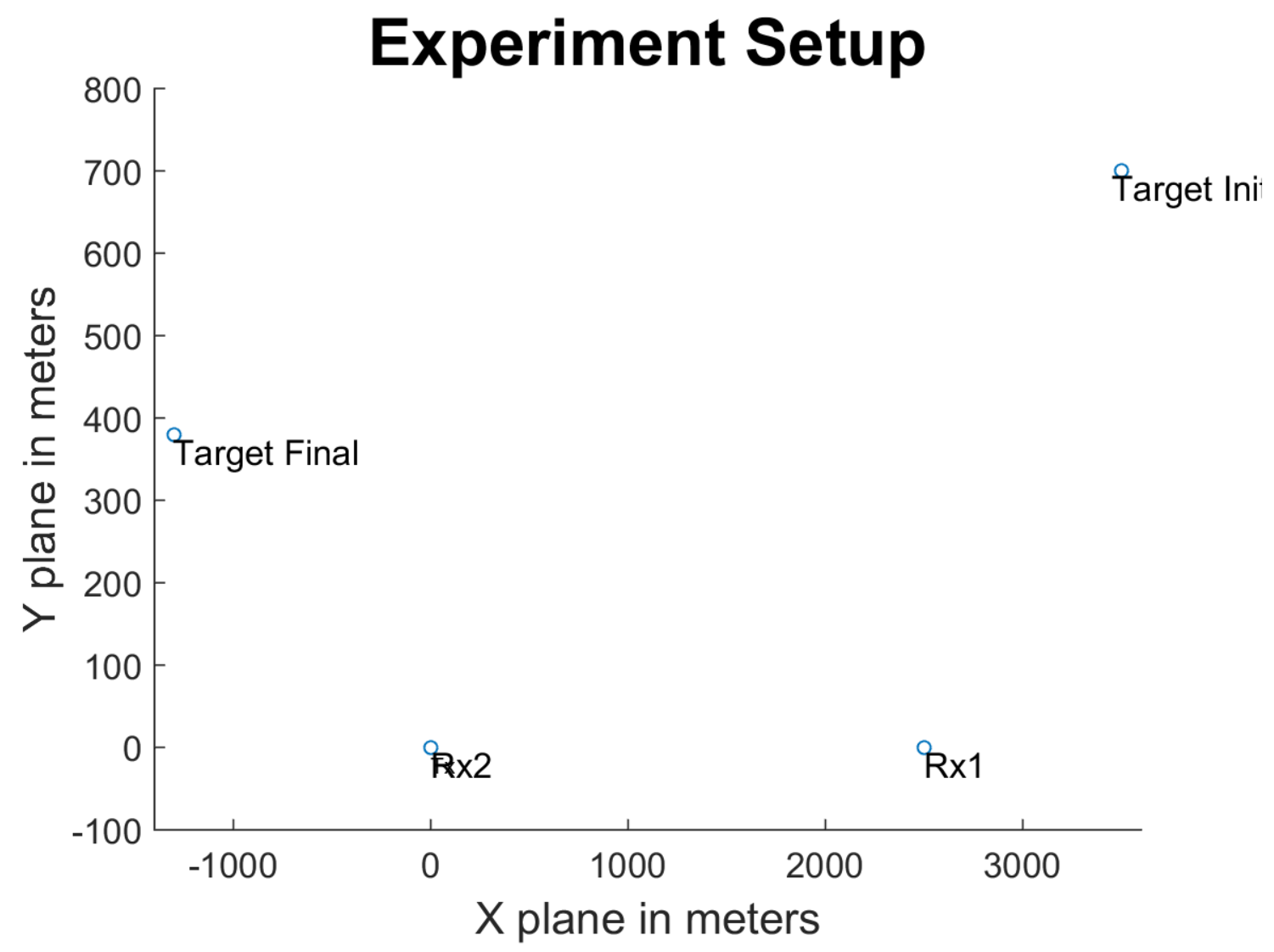

Figure 5-18 : Experimental setup for fixed noise demonstration using TDOA and FDOA

From Figure 5-19 we can see for the y-axis, the measurements get close to true value as GDOP improves between 15 to 25 seconds. Also the measurements compared to the actual data points are non-linear thus proving the non-linearity between the TDOA and FDOA measurements. 


\section{Fixed Noise}

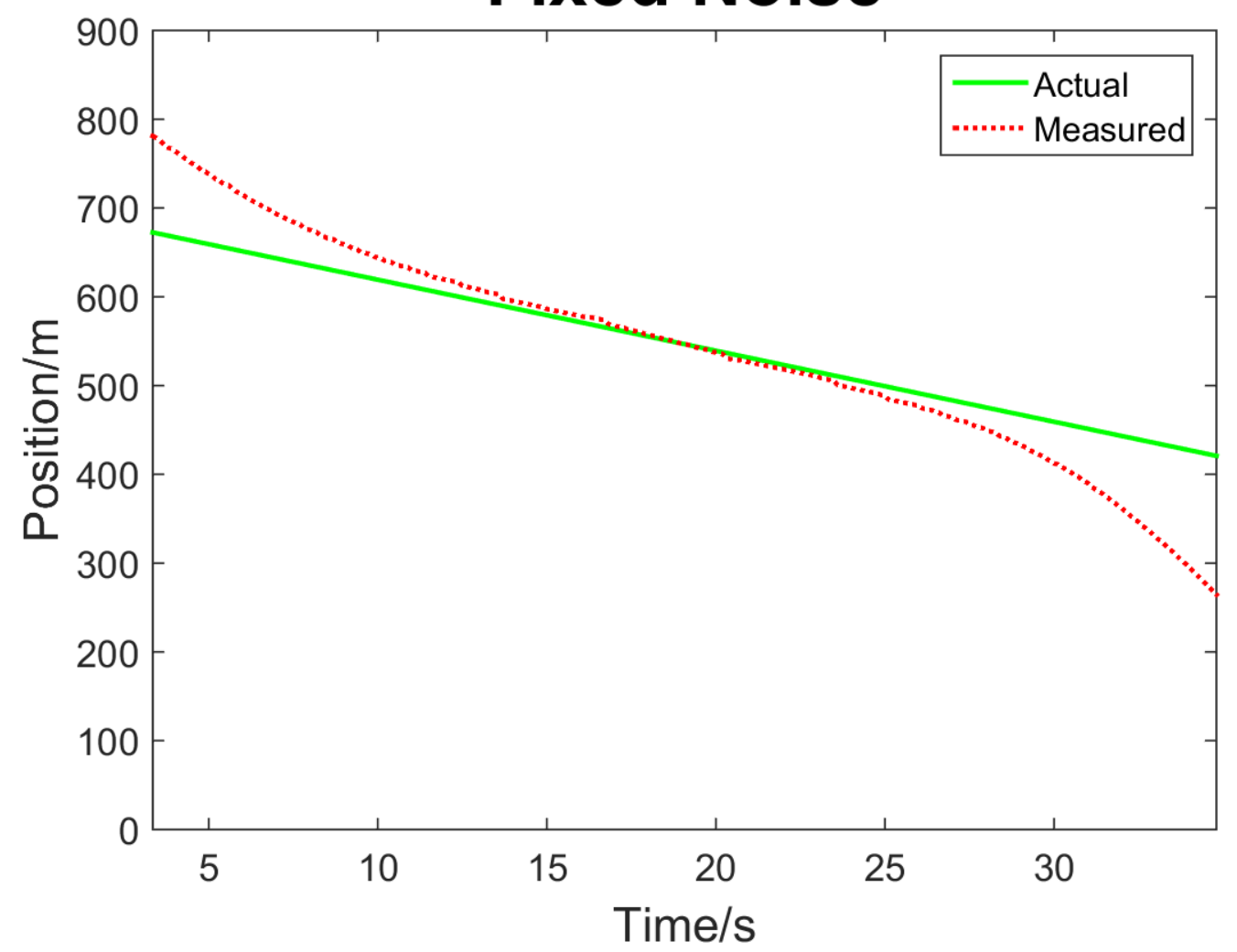

Figure 5-19: Y-axis measured location data for the TDOA and FDOA experiment with fixed noise

\subsection{Chapter Summary}

In this chapter we have covered the dual stage method for target geolocation of a moving target with unknown velocity and location. The velocity was then predicted using the TDOA multilateration technique and then the number of antennas used for the multistatic radar was reduced by one. This was made possible by introducing the FDOA multilateration technique in combination with the TDOA multilateration technique to locate the target. The best performance of locating the target with the lowest RMSE is 
when the GDOP is the best, which is when the target is within the multistatic radar baseline. 


\section{Chapter 6 Conclusion and Recommendations}

In this section, I will conclude the research and highlight results as part of the discussion. I will then present future work as part of the recommendations for this research.

\subsection{Conclusion and Discussion}

Intelligence Surveillance and Reconnaissance is a key element in modern warfare. Being able to gather intelligence to detect and locate the enemy while continuing to monitor the target in real time plays a key role in providing decision makers situational awareness.

With the use of the traditional monostatic radar today having the transmitter and receiver collocated, we encounter two major issues. The first is not having the capability to detect the stealth aircraft due to reduced radar cross section as observed by the radar receiver. Secondly, the monostatic radar can be located by the enemy using counter measure techniques hence revealing one's position and thus giving the enemy an advantage to bring down the radar surveillance capability.

A solution to this problem is the use of multistatic radar where receivers, transmitters and transceivers are scattered across a plane. The advantage of this is that the enemy cannot spot the location of the receivers. Also the use of readily available transmitters, such as radio broadcasts, reduces the cost of the radar setup and the possibility of being detected. Secondly, the stealth aircraft can now be detected with the distributed receiver antennas as part of the multistatic radar setup due to the fact that stealth aircraft deflect signals 
away from the point of source. This gives a larger SNR signal to be picked by receivers positioned around the target away from the transmitter.

The technique used to gather data from the multistatic radar setup is called the multilateration technique of which the Time Difference of Arrival and the Frequency Difference of Arrival are a part of this research to geolocate the stealth target.

The TDOA is measured by taking the difference in time the same signal coming from the target arrives at each pair of receivers. With a pulsed signal, the rising or falling edge of the signal can be used as a reference mark for the time of arrival. Taking the difference in the time of arrival gives rise to the time difference of arrival. The FDOA is measured using FFT. A peak in the spectrum will indicate the frequency of arrival, note there maybe harmonics that will have to be ignored. Taking the difference in the frequency of arrival between any two receivers gives rise to the frequency difference of arrival.

The modified Hough Transform is used to fuse two or more multilateration technique measurements and translate them to a Cartesian coordinate with a peak forming at the measured target location. The next target position is measured the same way except this time, because the target is in motion, the measurement will change to reflect the update in position. In order to keep track of the target and improve the SNR of the target location over multiple measurement samples, the Kalman Filter is used. The Kalman Filter helps in reducing the least squared error that can be observed through the reduction in root mean squared error. 
In this thesis, the first focus was on the hybrid geolocation and tracking of a moving stealth target where the initial target location and the velocity are unknown. The dual stage method was used to address this problem while using minimum number of receivers. Most of the extant literature (for example [9], [12]) covers geolocation of a static target or moving target with known velocity, using receiver sensors that move with known velocity in order to use the Doppler dependent multilateration technique such as the FDOA. The second focus was in transitioning from the use of three receivers to geolocate the target in a two dimensional space down to two receivers using the dual stage method. This ensures continued surveillance in the event one of three receivers goes down due to enemy attack or loses connection with central processing center.

Chapter 5 covers simulation on how a velocity estimate is formed using three receiver antennas with the TDOA multilateration technique. In order for the measurement of the target location to take place, a range of distance has to be provided for the voting array covered in Chapter 3. The target will not be geolocated if this voting array lies outside the target location plus or minus noise. The Kalman Filter was used to refine the target position, hence velocity estimation was computed. With the velocity prediction remaining fairly steady (assuming velocity is constant), we arrive at the second stage. Now, the use of the FDOA multilateration technique can take place as it is a function of velocity and hence the dual stage method allows for the transition down to two receiver antennas. This is because the TDOA and FDOA multlateration techniques provide lines of position that are orthogonal. 
Simulation results indicate that the FDOA multilateration technique is sensitive not only to frequency of arrival measurement noise but also to the estimated velocity from the prior stage. This is evident in the downrange RMSE of the location measurement. We can also see the effect of geometric dilution of precision or GDOP on measurement noise. As long as the target remains within the multistatic radar baseline we have good GDOP. This shows that if we want a larger area of surveillance coverage, we need a wider baseline defined by distance between receiver pairs.

\subsection{Recommendations}

One of the best ways of gathering intelligence today is by using passive radar techniques. This not only ensures enemy awareness but it allows one to continuously monitor without enemy counter measure disruptions. This, however, poses challenges and I have demonstrated in this thesis with assumptions a method to geolocate a stealth target in a two dimensional space.

It would be interesting to implement this thesis in three dimensions and see how the dual stage method may be applied to reduce the number of receiving antennas. It would also be interesting to see if other multilateration technique(s) such as angle of arrival, could be used to enhance measured data at each target location.

The choice of wavelength has not been studied in reference to detecting stealth aircraft such that we see the highest RCS and yet being optimal to be used by multistatic radar for the purposes of different multilateration techniques such as time of arrival, frequency of arrival and angle of arrival. 
A Gaussian Measurement Mixture filter should be explored where passive measurements taken due to multilateration techniques such as TDOA and FDOA cause a non-Gaussian distribution in the observation space. Hence this filter will model any non-Gaussian distribution as a Gaussian mixture. This is because the Hough Transform is computationally intensive.

A feasibility study on synchronization and transfer of received signals from each receiver to be processed at a central station should be explored. The delays introduced due to transfer and processing of the signal may require reduction in the sampling size and thus effect the accuracy of filtered target geolocation.

Furthermore, instead of making various assumptions, like in this research, which does not paint a detailed picture, a more realistic model not bound with assumptions would enhance the experimental model. Finally, by performing the experiment with multiple targets, non-line of sight, effects of clutter and by introducing various noise sources into the model such as antenna receiver, a more realistic model would be arrived at from which further analysis could be conducted. 


\section{Appendices}

\section{Appendix A Matlab TDOA and FDOA Extraction}

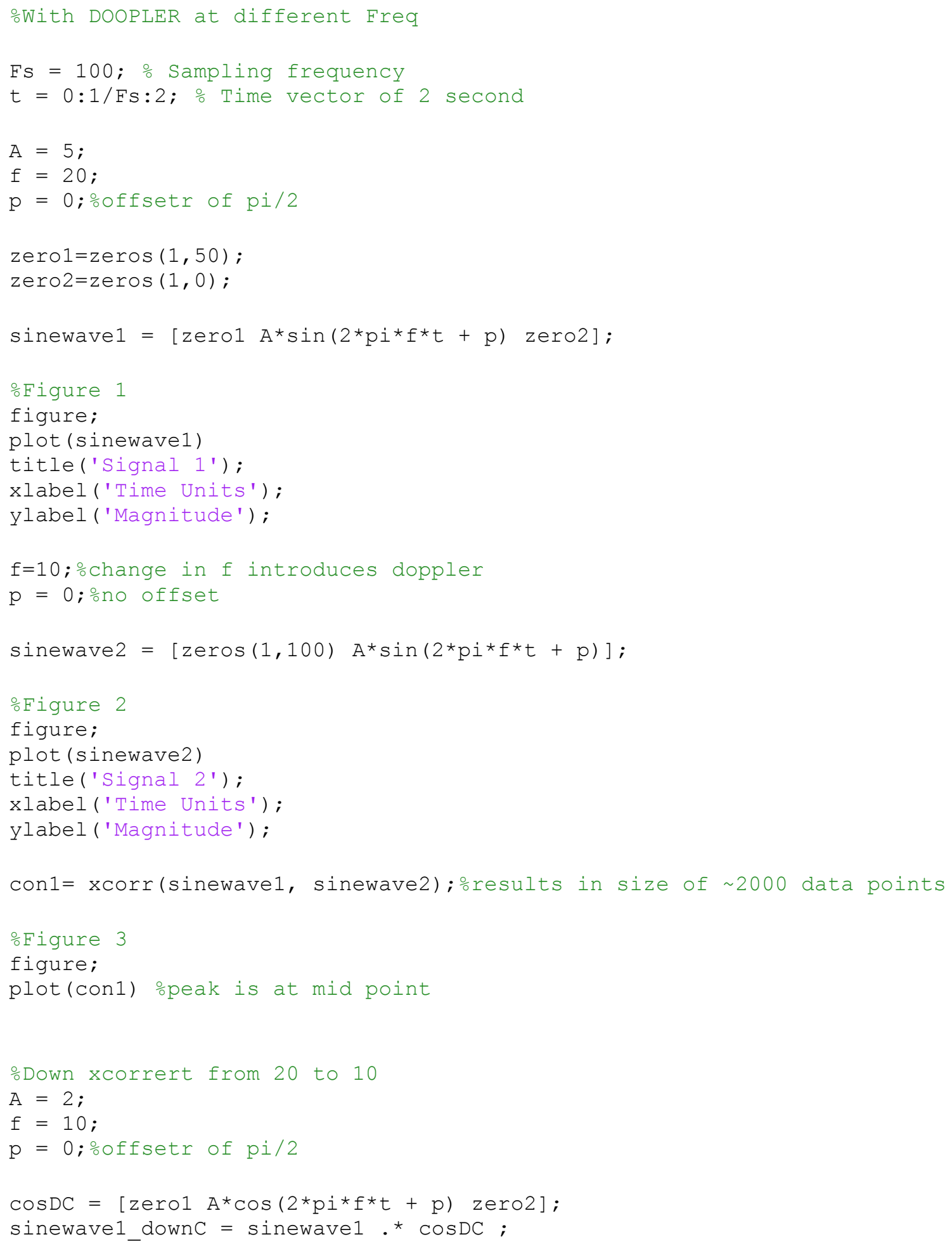




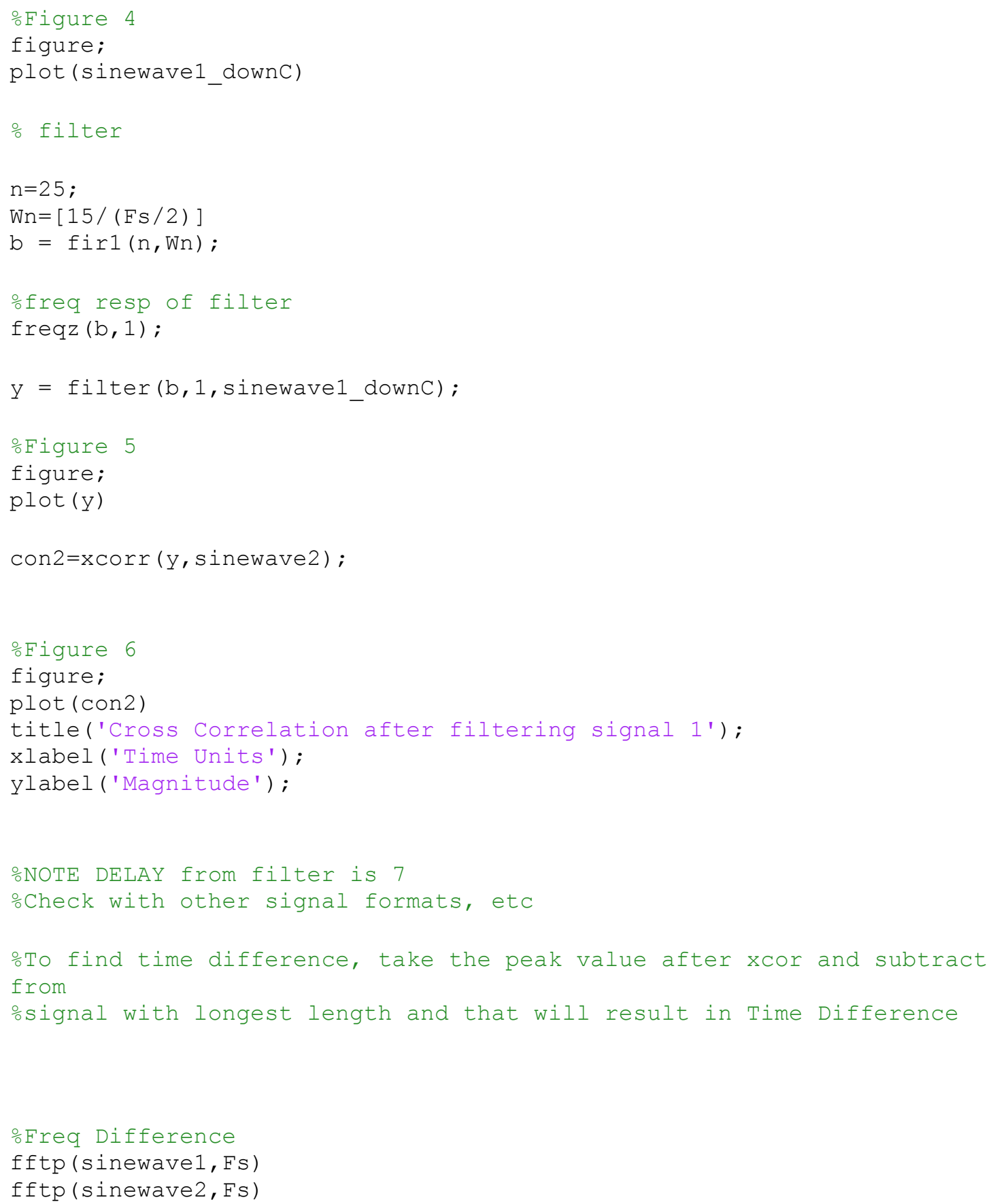

function []$=f f t p$ (signal, Fs)

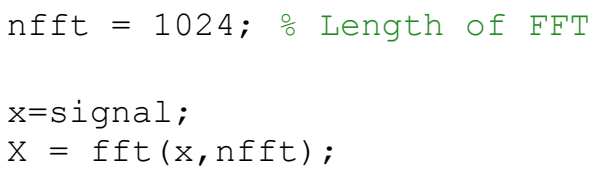




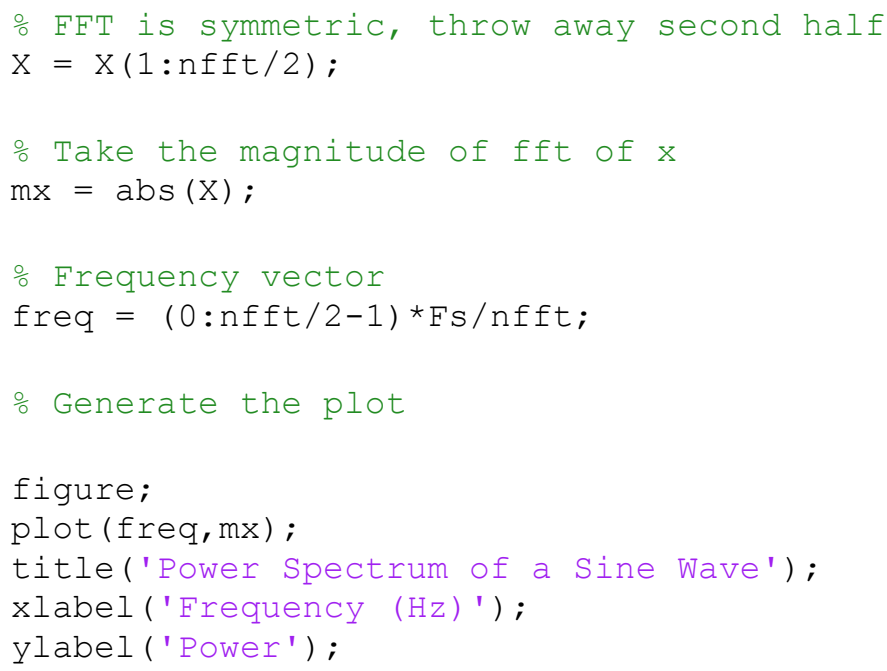

end 


\section{Appendix B Matlab Kalman Filter}

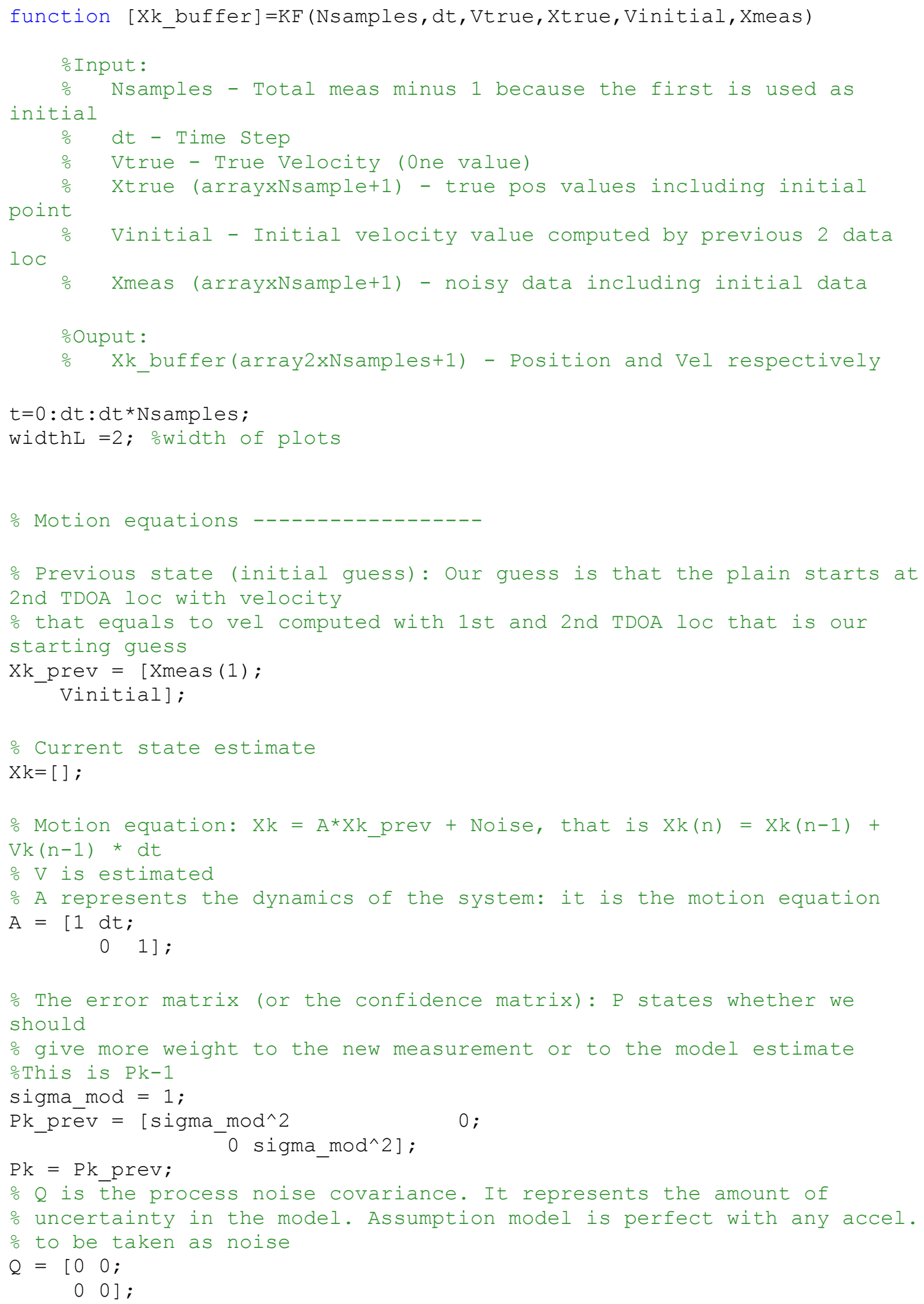




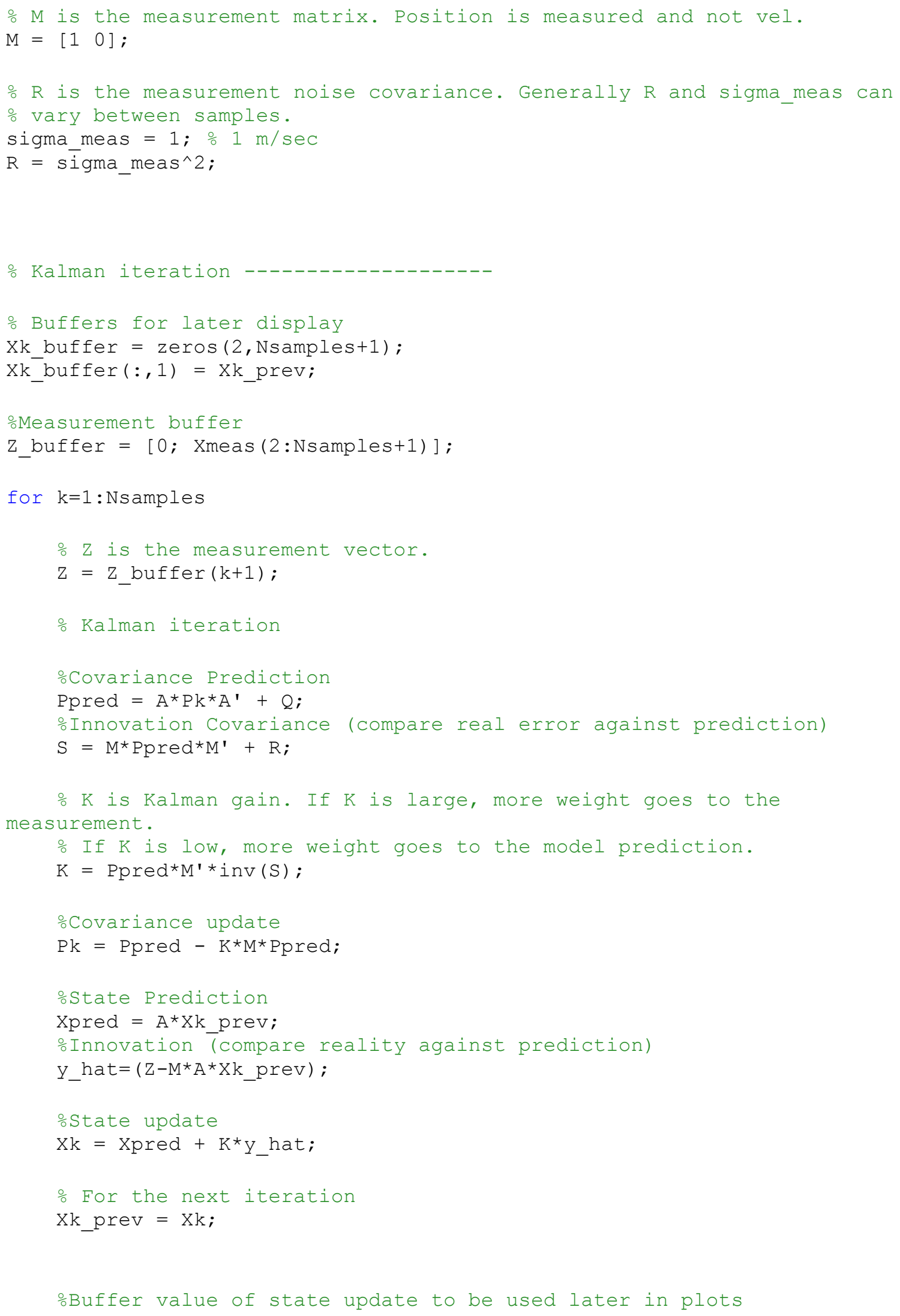


Xk_buffer $(:, \mathrm{k}+1)=\mathrm{Xk}$;

end;

○Graphs

figure;

plot (t, Xtrue, 'g', 'LineWidth', widthL); hold on;

plot (t,z_buffer,'r', 'LineWidth', widthL);

plot ( $t, x \bar{k}$ buffer $(1,:)$, 'b' ' 'LineWidth', widthL);

legend('Actual', 'Measured', 'KF');

title('Predicted position results');

xlabel ('Time/s');

ylabel ('Position/m');

figure;

plot (t, ones (size(t)) *Vtrue, 'g', 'LineWidth', widthL); hold on;

plot (t,Xk_buffer (2, :), 'b', 'LineWidth', widthL);

legend('Actual velocity','KF');

title('Predicted velocity results');

xlabel ('Time/s');

ylabel ('Velocity/(m/s)');

end 


\section{Appendix C Matlab Experiment Model}

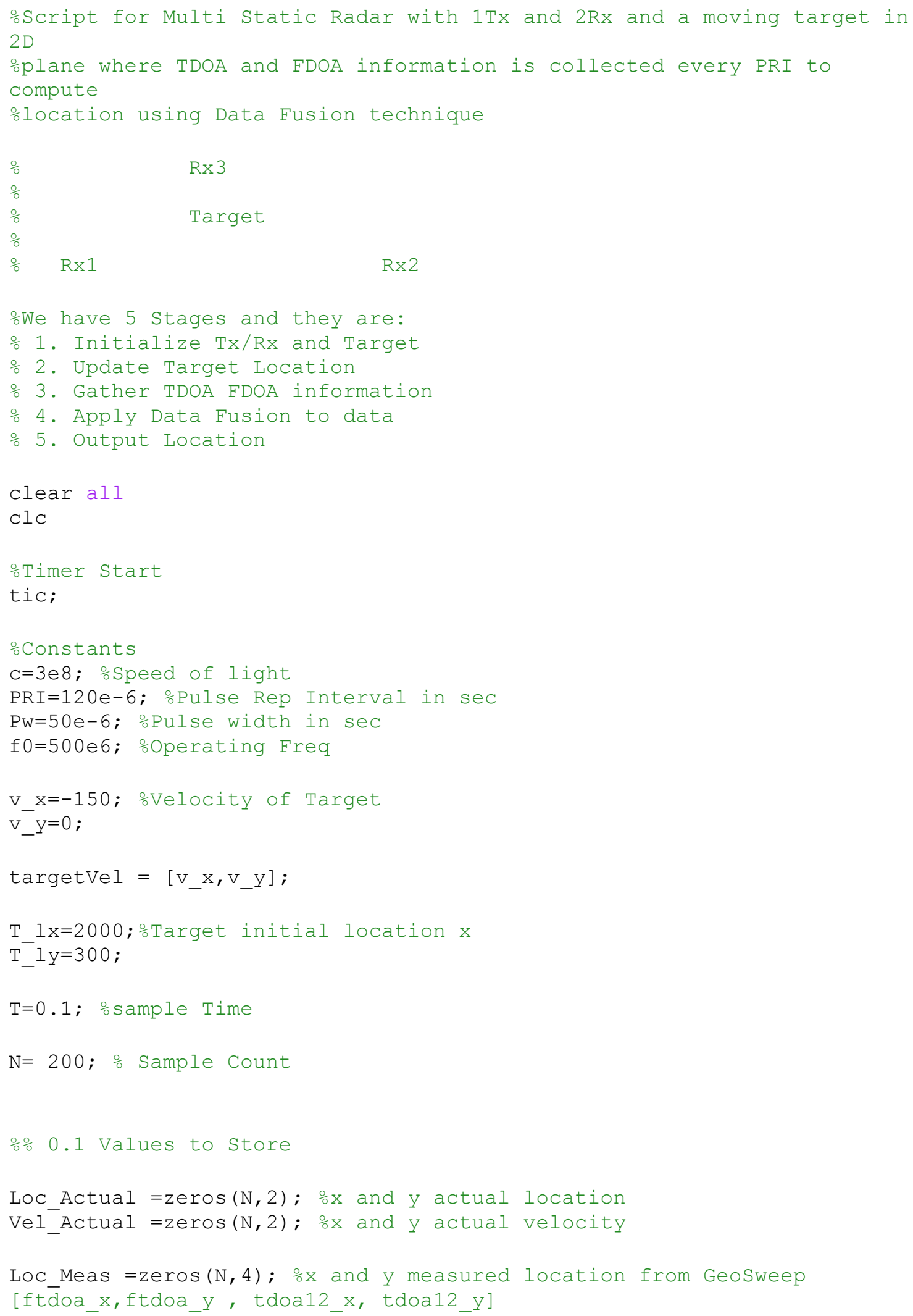




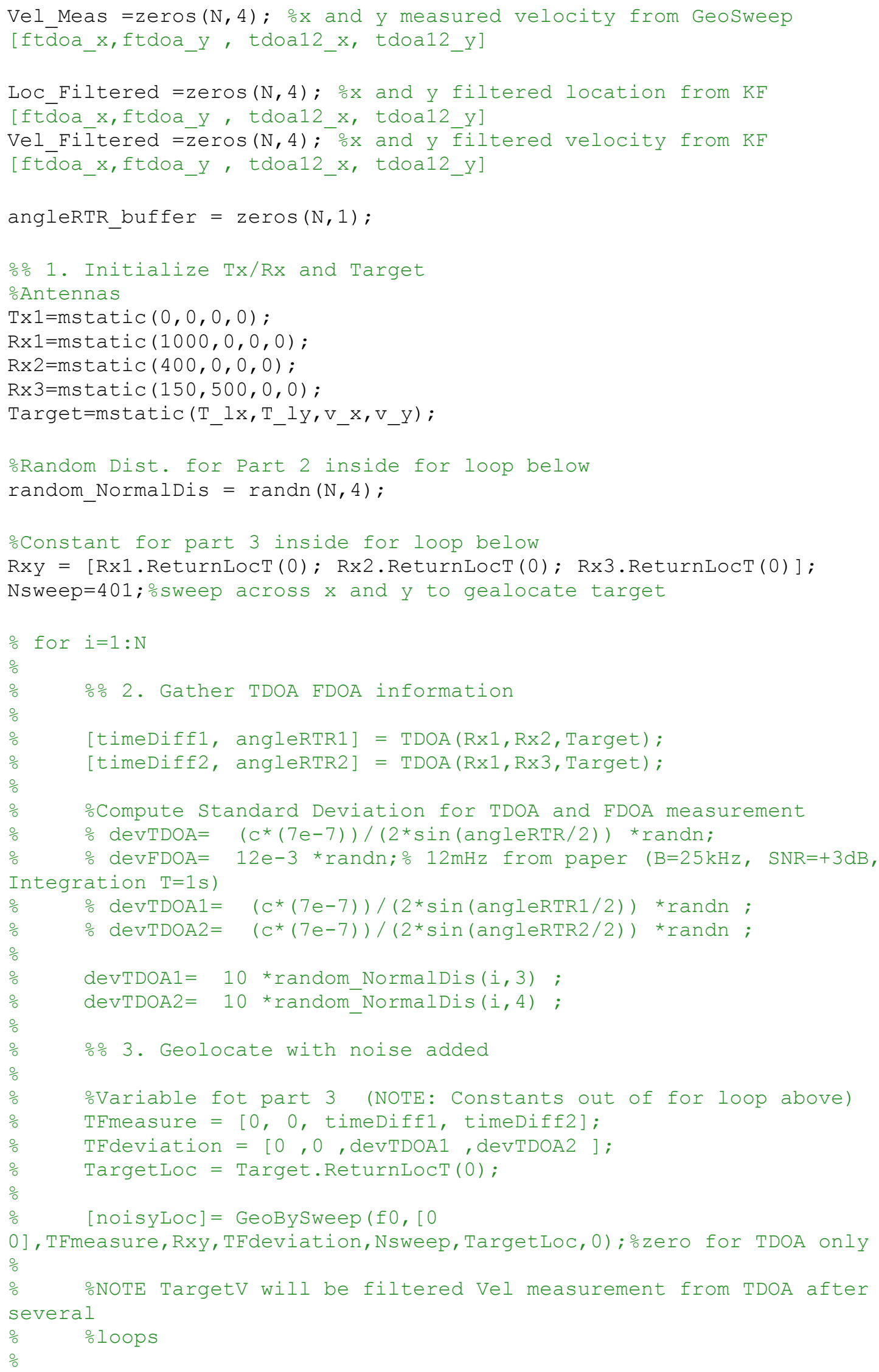




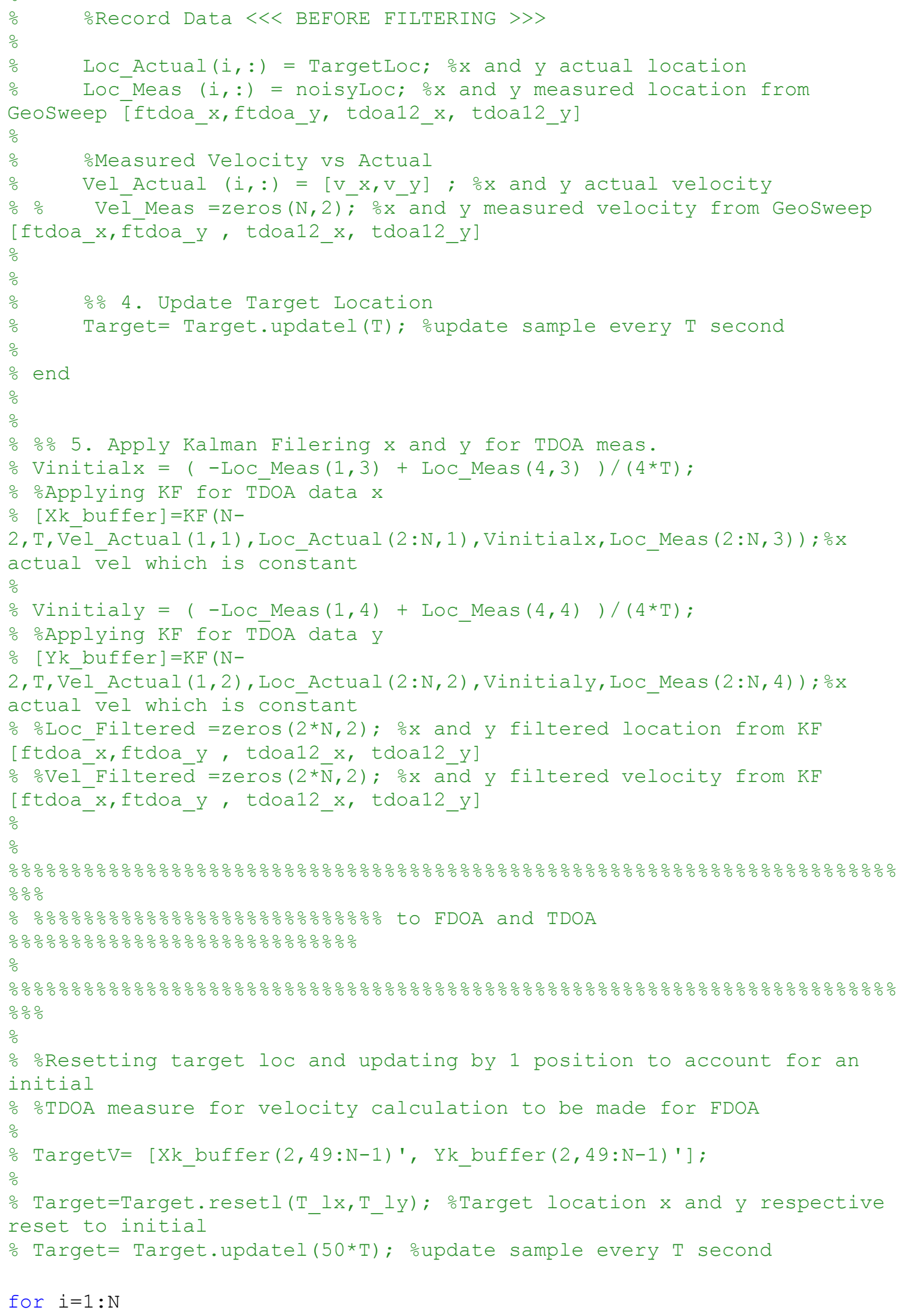




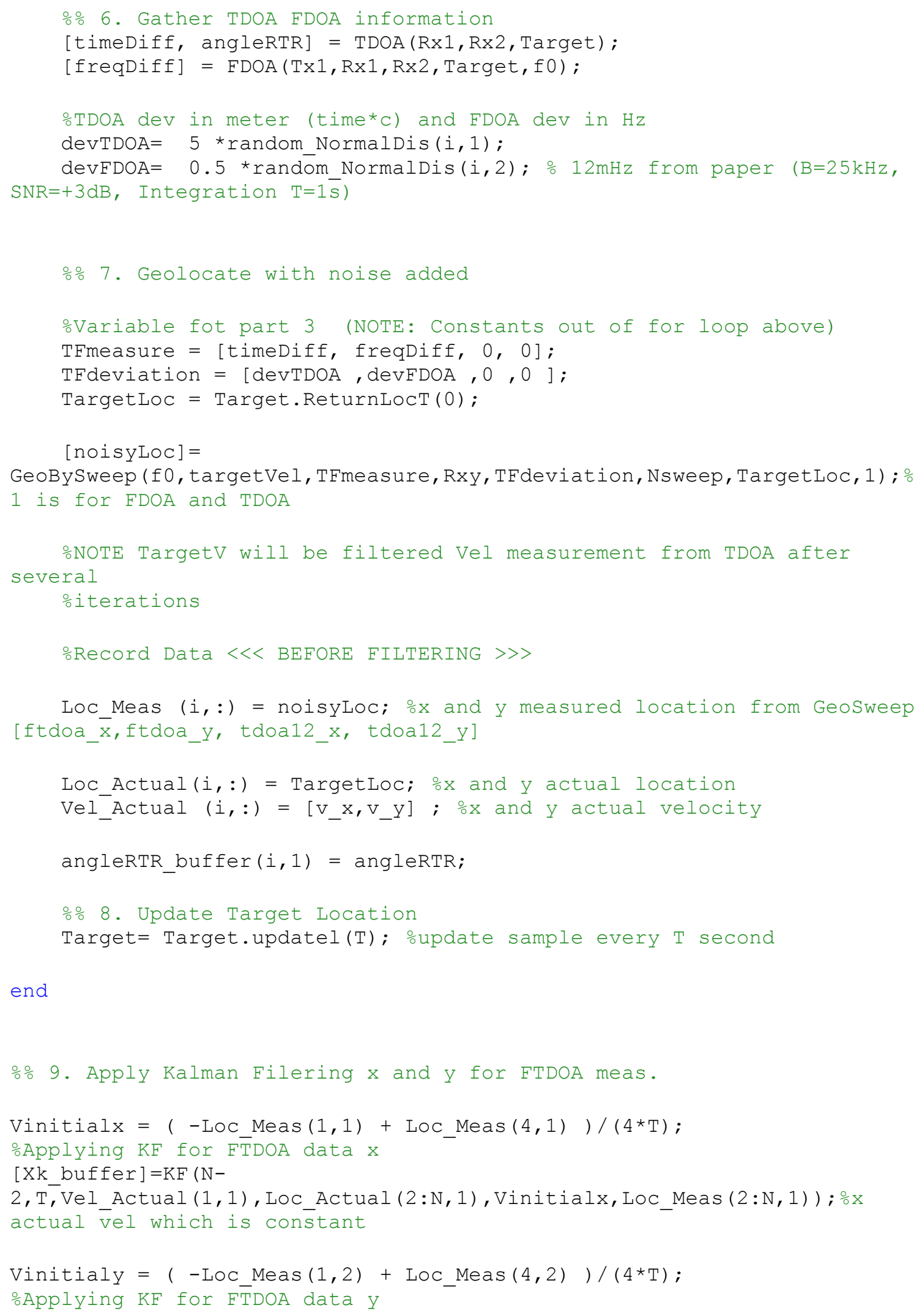


$[\mathrm{Yk}$ _buffer] $=\mathrm{KF}(\mathrm{N}-$

2, T, Vel Actual $(1,2), \operatorname{Loc}$ Actual $(2: \mathrm{N}, 2), \operatorname{Vinitialy,~Loc~Meas~}(2: \mathrm{N}, 2))$; 응 actual vel which is constant

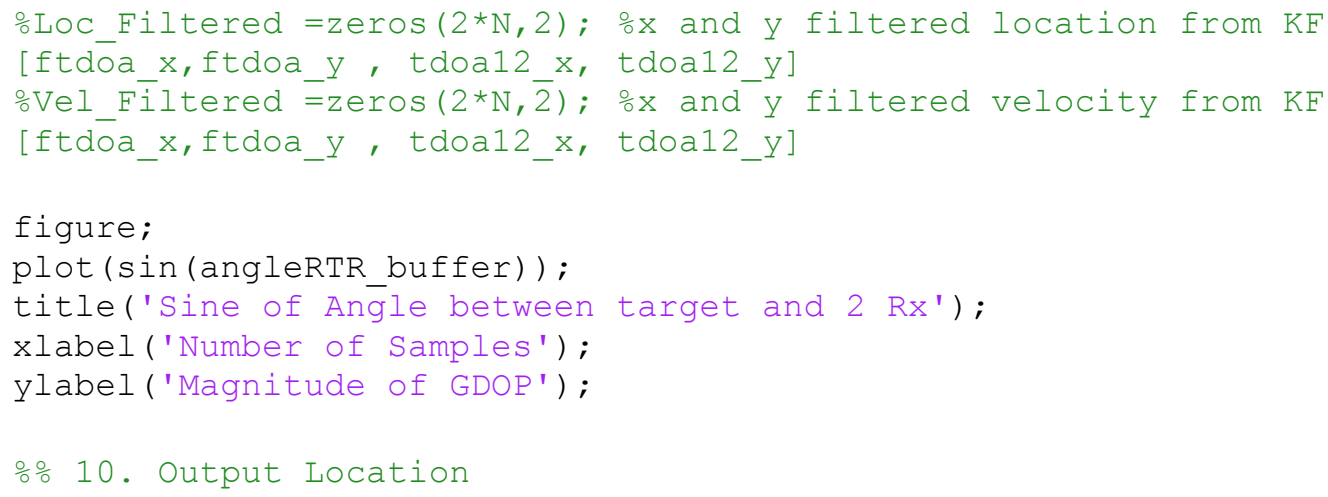




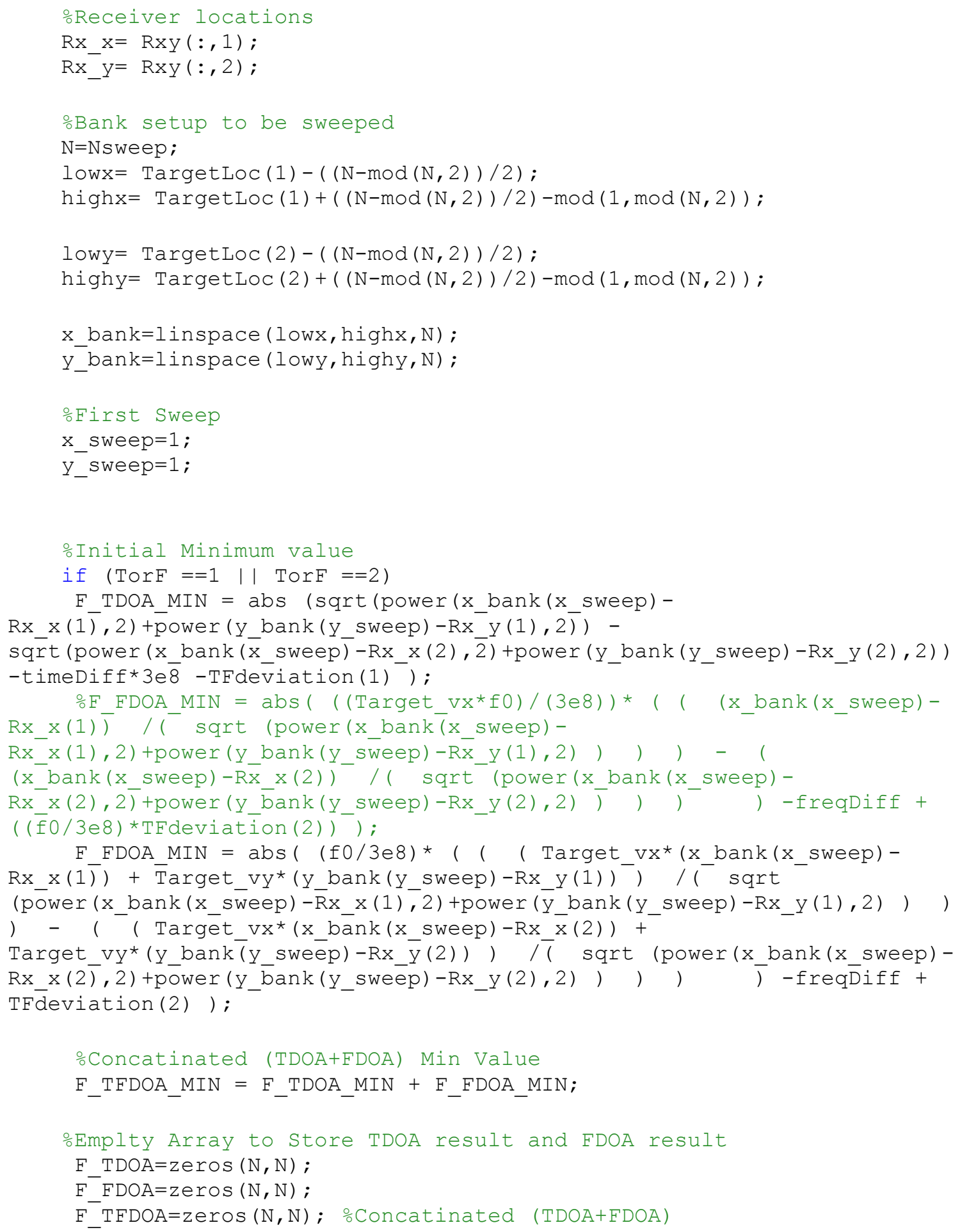


F TDOA MIN1 = abs $(\operatorname{sqrt}(\operatorname{power}(\mathrm{x} \operatorname{bank}(\mathrm{x}$ sweep) $\operatorname{Rx} x(1 \overline{)}, 2)+\bar{p}$ ower $(y \operatorname{bank}(y$ sweep $)-\operatorname{Rx} \bar{y}(1), 2) \overline{)}$

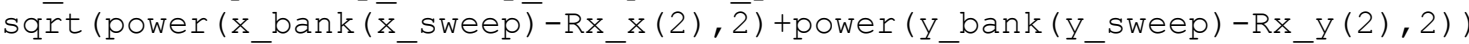
-timeDiff1*3ē8 -TFdēiation (3) );

F_TDOA_MIN2 = abs (sqrt (power $\left(x \_b a n k\left(x \_s w e e p\right)-\right.$

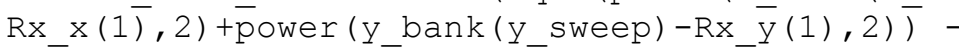

sqūt (power (x_bank ( $\bar{x}$ sweep) $-\operatorname{Rx} x(3), \overline{2})$ +power (y_bank (y_sweep) -Rx_y (3), 2))

-timeDiff $2 * 3 \bar{e} 8-T F d \bar{e} v i a t i o n(4 \overline{)})$;

oConcatinated (TDOA+FDOA) Min Value

F_TDOA_MIN12 = F_TDOA_MIN1 + F_TDOA_MIN2;

Emplty Array to store TDOA result and FDOA result

$\mathrm{F} \operatorname{TDOA} 1=\operatorname{zerOs}(\mathrm{N}, \mathrm{N})$;

$\mathrm{F}$ TDOA2 $=$ zeros $(\mathrm{N}, \mathrm{N})$;

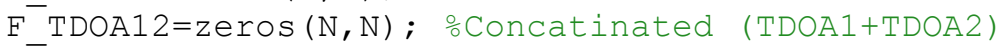

end

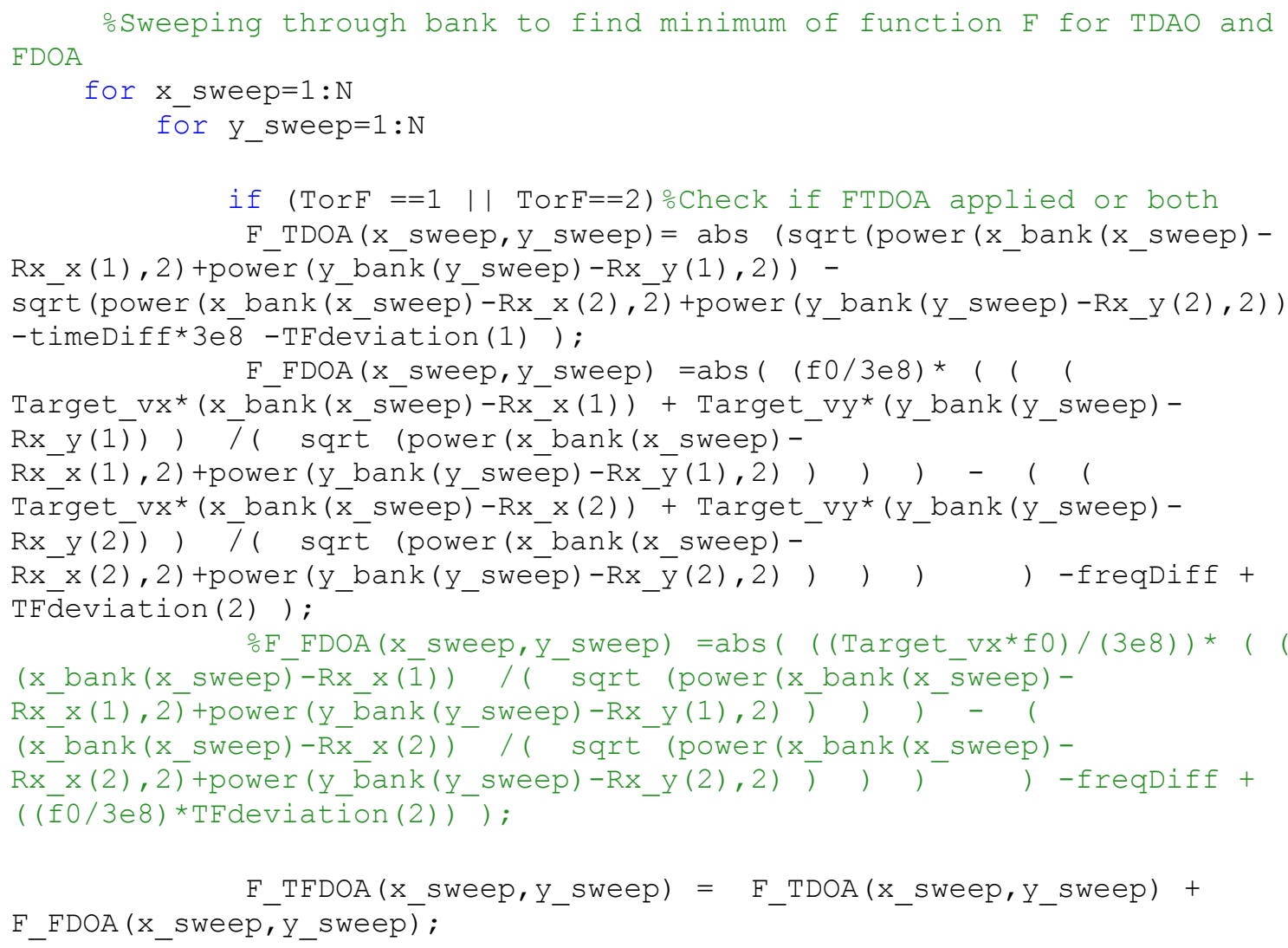




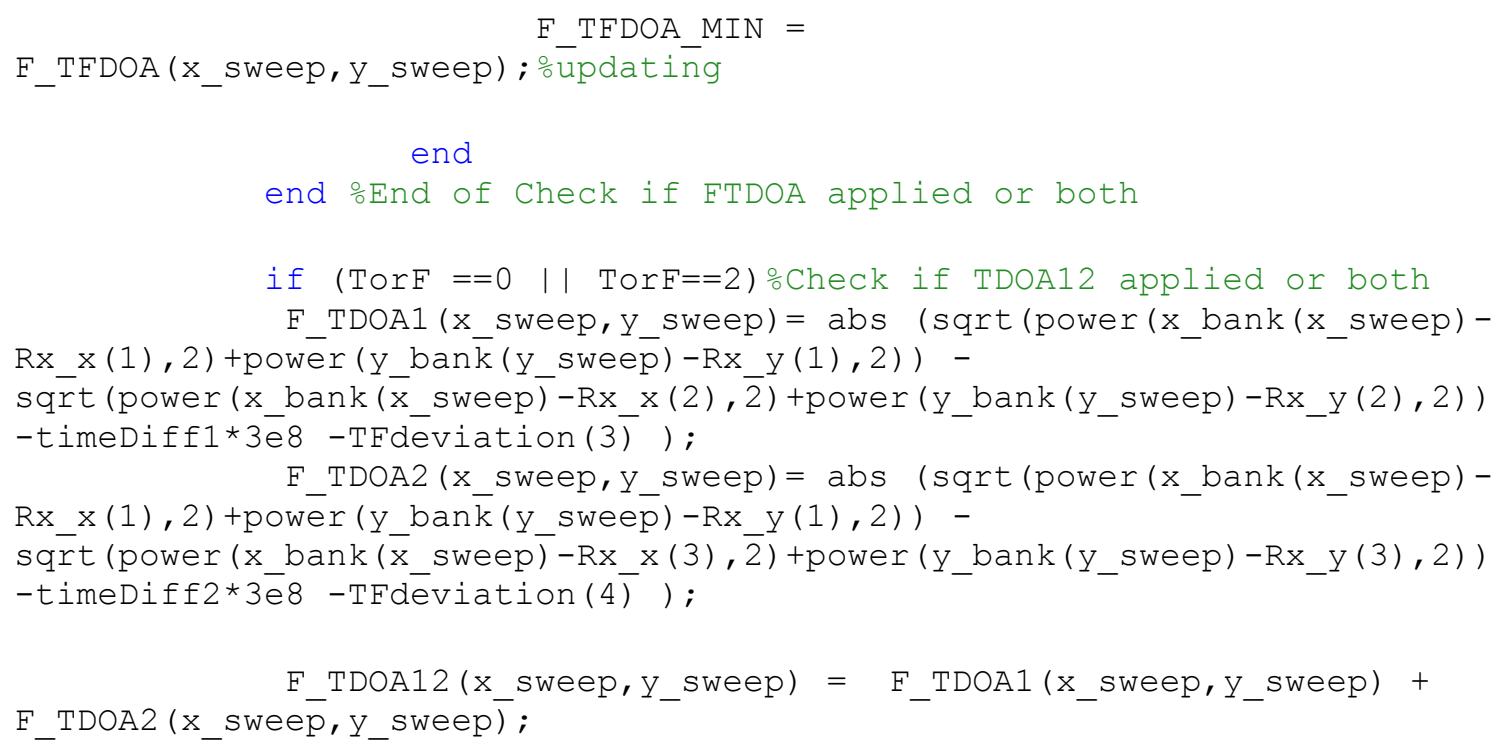




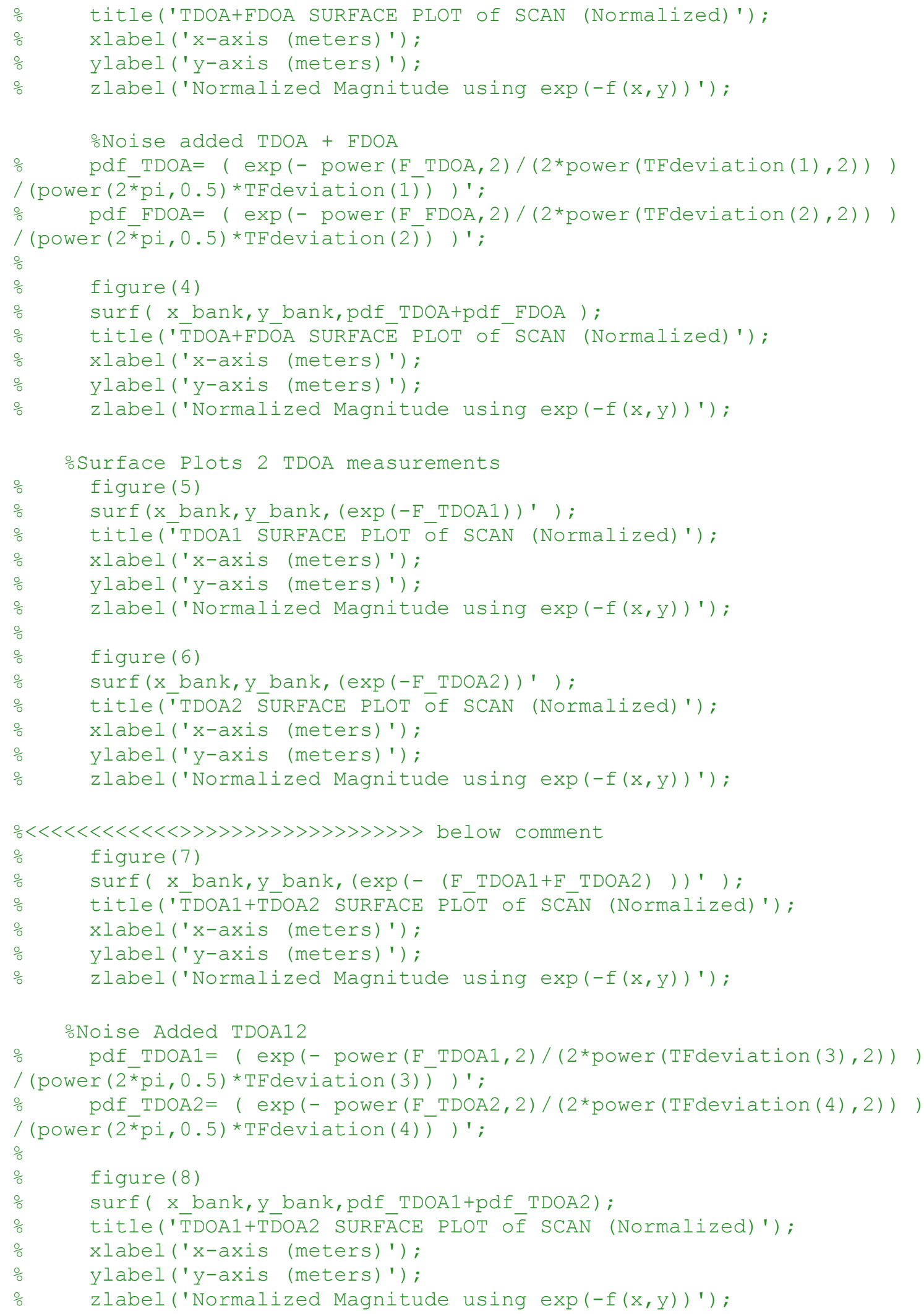




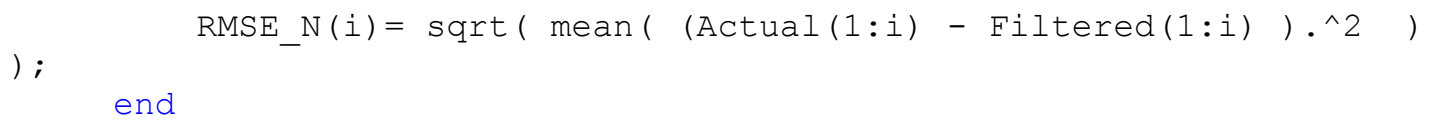




\section{Bibliography}

1. Shawn R. Doughty, "Development and Performance Evaluation of a Multistatic Radar System", Ph.D. Thesis, University of London, 2008

2. Nicholas J. Willis, Hugh D. Griffiths, Advances in Bistatic Radar, SciTech, 2007

3. Richard Poisel, Electronic Warfare Target Location Methods, Artech House Inc, 2012

4. Richard G. Wiley, ELINT: The Interception and Analysis of Radar Signals, Artech House, 2006

5. Merrill I. Skolnik, David J. Murrow, Radar Handbook, McGraw-Hill, 1990

6. Konstantinos Zikidis, Alexios Skondras, Charisios Tokas, "Low Observable Principles, Stealth Aircraft and Anti-Stealth Technologies", Journal of Computations \& Modelling, vol.4, 2014

7. Nicholas J. Willis, Bistatic Radar, SciTech 2005

8. William L. Melvin and James A. Scheer, Principles of Modern Radar: Radar Applications, The Institution of Engineering and Technology, 2013

9. Darko Musicki, Wolfgang Koch, "Geolocation using TDOA and FDOA Measurements”, Watchtberg, Germany, 2008

10. [Commander Josh Barber, “An Intelligence Surveillance and Reconnaissance (ISR) Vision for the Canadian Forces", Canadian Military Journal, 2002

11. Kimberly N. Hale, "Expanding the Use of Time/Frequency Difference of Arrival Geolocation in the Department of Defense", Ph.D. Dissertation, Pardee RAND Graduate School, California, 2012 
12. A. Mikhalev, "Image Processing and Agent-Based framework for the Geolocation of Emitters", Ph.D. Thesis, Cranfield University, U.K., 2009

13. Volkan Tas, "Optimal Use of TDOA Geo-Location Techniques Within the Mountainous Terrain of Turkey", Master Thesis, Naval Post Graduate School, California, 2012

14. William L. Melvin and James A. Scheer, Principles of Modern Radar: Basic Principles, SciTech, 2010

15. Knapp, C., \& Carter, G., "The generalized correlation method for estimation of time delay", IEEE Transactions on Acoustics, Speech and Signal Processing, Vol 24, 1976

16. Christopher L. Yatrakis, "Computing the Cross Ambiguity Function - A Review". Master Thesis, Binghamton University and State University of New York, 2005

17. Maheshwaran Subramaniam, "Multitarget Tracking Using Multistatic Sensors", Ph.D. Thesis, McMaster University, 2012

18. Yuan Jiang, Ran Xia, Jia Xu, Quanhua Liu, Xinliang Chen, "Multiple Aircraft Formation Identification using OMP-Based Time-Frequency Analysis and Hough Transform”, Shanghai University, China, 2015

19. Richard B. Langley, "Dilution of Precision”, University of New Brunswick, 1999

20. John W. Franklin, “Passive Bistatic Radar”, 2010

21. William D. Blair, "Radar Tracking”, Georgia Tech Research Institute, IEEE Radar Conference, 2013 